DRAFT VERSION MARCH 19, 2019

Preprint typeset using LTEX style emulateapj v. 12/16/11

\title{
SOFIA FORCAST PHOTOMETRY OF 12 EXTENDED GREEN OBJECTS IN THE MILKY WAY
}

\author{
A. P. M. Towner ${ }^{1 * 2}$, C. L. Brogan ${ }^{1}$, T. R. Hunter ${ }^{1}$, C. J. CYGANOWSKi ${ }^{3}$, R. K. Friesen ${ }^{1}$ \\ Draft version March 19, 2019
}

\begin{abstract}
Massive young stellar objects are known to undergo an evolutionary phase in which high mass accretion rates drive strong outflows. A class of objects believed to trace this phase accurately is the GLIMPSE Extended Green Object (EGO) sample, so named for the presence of extended $4.5 \mu \mathrm{m}$ emission on sizescales of $\sim 0.1$ pc in Spitzer images. We have been conducting a multi-wavelength examination of a sample of 12 EGOs with distances of 1 to $5 \mathrm{kpc}$. In this paper, we present mid-infrared images and photometry of these EGOs obtained with the SOFIA telescope, and subsequently construct SEDs for these sources from the near-IR to sub-millimeter regimes using additional archival data. We compare the results from greybody models and several publicly-available software packages which produce model SEDs in the context of a single massive protostar. The models yield typical $R_{\star} \sim 10 R_{\odot}, T_{\star} \sim 10^{3}$ to $10^{4} \mathrm{~K}$, and $L_{\star} \sim 1-40 \times 10^{3} L_{\odot} ;$ the median $L / M$ for our sample is $24.7 L_{\odot} / M_{\odot}$. Model results rarely converge for $R_{\star}$ and $T_{\star}$, but do for $L_{\star}$, which we take to be an indication of the multiplicity and inherently clustered nature of these sources even though, typically, only a single source dominates in the mid-infrared. The median $L / M$ value for the sample suggests that these objects may be in a transitional stage between the commonly described "IR-quiet" and "IR-bright" stages of MYSO evolution. The median $T_{\text {dust }}$ for the sample is less conclusive, but suggests that these objects are either in this transitional stage or occupy the cooler (and presumably younger) part of the IR-bright stage.
\end{abstract}

Subject headings: stars: formation - stars: massive - stars: protostars - infrared: general - radiative transfer techniques: photometric

\section{INTRODUCTION}

Massive young stellar objects (MYSOs) are challenging to observe due to their comparative rarity and short-lived natal phase, large distances from Earth, and highly-obscured formation environments. Early observations of suspected MYSOs were performed mostly with large beams, and probed size scales ranging from cores to clumps and clouds $(\sim 0.1 \mathrm{pc}$, $\sim 1 \mathrm{pc}$, and $\sim 10 \mathrm{pc}$, respectively; see Kennicutt \& Evans 2012). Detailed descriptions of early surveys for MYSOs and their results can be found in, e.g., Molinari et al. (1996), Sridharan et al. (2002), and Fontani et al. (2005). Followup observations with improved sensitivity and spatial resolution, such as interferometric radio and millimeter observations, revealed that many of the objects originally identified as "MYSOs" were actually sites in which multiple protostars were forming simultaneously (e.g. Hunter et al. 2006 Cyganowski et al.2007; Vig et al. 2007, Zhang et al. 2007, to name just a few). This predilection for forming in clustered environments means that the study of high-mass protostars is necessarily the study of protoclusters: clusters of protostars with a range of masses and in a variety of evolutionary stages. Current theories of high mass star formation differ in their predictions of the aggregate properties of these protoclusters, such as mass segregation (if any), sub-clustering of the protostars, and stellar birth order (e.g. Vázquez-Semadeni et al. 2017; Banerjee \& Kroupa 2017; Bonnell \& Bate 2006; McKee \& Tan 2003). It is therefore necessary to consider each

\footnotetext{
${ }^{1}$ National Radio Astronomy Observatory, 520 Edgemont Rd, Charlottesville, VA 22903, USA

${ }^{2}$ Department of Astronomy, University of Virginia, P.O. Box 3818, Charlottesville, VA 22903, USA

${ }^{3}$ Scottish Universities Physics Alliance (SUPA), School of Physics and Astronomy, University of St. Andrews, North Haugh, St Andrews, Fife KY16 9SS, UK

A.P.M.T. is a Grote Reber Doctoral Fellow at the National Radio Astronomy Observatory.
}

high-mass protostar in combination with its environment.

Extended Green Objects (EGOs) were first identified by Cyganowski et al. (2008) using data from the Galactic Legacy Infrared Midplane Survey Extraordinaire (GLIMPSE, Benjamin et al. 2003; Churchwell et al. 2009) project. EGOs are named for their extended emission in the $4.5 \mu \mathrm{m}$ Spitzer IRAC band (commonly coded as "green" in three-color RGB images), which is due to shocked $\mathrm{H}_{2}$ from powerful protostellar outflows (e.g., Marston et al. 2004). Follow-up observations of $\sim 20$ EGOs with the Karl G. Jansky Very Large Array (VLA) by Cyganowski et al. (2009) established both the presence of massive protostars (traced by $6.7 \mathrm{GHz}$ Class II $\mathrm{CH}_{3} \mathrm{OH}$ masers) and shocked molecular gas indicative of outflows (traced by $44 \mathrm{GHz}$ Class I $\mathrm{CH}_{3} \mathrm{OH}$ masers). The causal link between accretion and ejection (Frank et al. 2014) thus implies that these objects contain protostars undergoing active accretion, and the maser data indicate that these protostars are massive. The youth of the massive protostars within these EGOs was confirmed by deep (at that time) VLA continuum observations (Cyganowski et al. 2011b), which yielded only a few $3.6 \mathrm{~cm}$ detections, and by later VLA $1.3 \mathrm{~cm}$ continuum observations (Towner et al. 2017), which revealed primarily weak $\left(<1 \mathrm{mJy} \mathrm{beam}^{-1}\right)$, compact emission. The low detection rates and integrated flux densities of the centimeter continuum emission in these sources demonstrate that any free-free emission is weak, consistent with a stage prior to the development of ultracompact HII regions.

Given that high-mass stars form in clusters, it is likely that EGOs are signposts for protoclusters rather than isolated high-mass protostars, though the level of multiplicity of massive sources $\left(>8 M_{\odot}\right)$ and overall cluster demographics remain open questions. Millimeter dust continuum observations of EGOs with $\sim 3^{\prime \prime}$ resolution, suggest that the number of massive protostars per EGO is typically one to a few (e.g. Cyganowski et al. 2012, 2011a; Brogan et al. 2011). How- 
ever, the precise physical properties of protoclusters traced by EGO emission - such as total mass, luminosity, and massive protostellar multiplicity - remain largely unexplored in EGOs as a class.

The infrared emission from EGOs, and indeed MYSOs in general, is often challenging to characterize due to the presence of high extinction from their surrounding natal clumps (as they are still deeply embedded), and confusion from more evolved sources nearby. The latter issue has been particularly affected by the relatively poor angular resolution $\left(>1^{\prime}\right)$ that has heretofore been available at mid- and far-infrared wavelengths, where the high extinction can be overcome. Yet these wavelengths contain crucial information as hot dust, shocked gas, and polycyclic aromatic hydrocarbons (PAHs) all emit in this regime. Scattered light originating from the protostar itself may also sometimes escape through outflow cavities and would likewise be visible in the infrared. Thus mid-infrared wavelengths are a crucial component of the Spectral Energy Distribution (SED) which is a useful tool for constraining important source properties such as mass, bolometric luminosity, and temperature.

These properties are of particular interest for MYSOs, as recent analysis of the Herschel InfraRed Galactic Plane Survey (Elia et al.2017) and a full census of the properties of ATLASGAL Compact Source Catalog (CSC) objects Urquhart et al. (2018) shows how the luminosity to mass ratio $L / M$ of protostellar clumps can be used to both qualitatively and quantitatively discriminate between the different evolutionary stages of pre- and protostellar objects. In theoretical terms, $L / M$ is tied to evolutionary state primarily due to abrupt changes in luminosity during different stages of MYSO/clump evolution (see, e.g., the stages described in Hosokawa \& Omukai 2009. Molinari et al. 2008).

In this paper, we present new data that directly address the questions of the multiplicity and physical properties (temperature, mass, and luminosity) of the massive protoclusters traced by EGOs. We have utilized the unique capabilities of the Stratospheric Observatory for Infrared Astronomy (SOFIA, Temi et al.2014) to image a well-studied sample of 12 EGOs at two mid-IR wavelengths: 19.7 and $37.1 \mu \mathrm{m}$ with the necessary sensitivity $\left(\sim 0.05\right.$ to $\sim 0.25 \mathrm{Jy}^{\text {beam }^{-1}}$ ) and angular resolution $\left(\sim 3^{\prime \prime}\right)$ to detect and resolve the mid-infrared emission from the massive protocluster members. By combining these results with ancillary multi-wavelength archival data, we create well-constrained SEDs from the near-infrared through submillimeter regimes. We then use three SED modelling packages published by Robitaille et al. (2006), Robitaille (2017), and Zhang \& Tan (2018), to constrain physical parameters (see, e.g., Gaczkowski et al. 2013; De Buizer et al. 2017). In $\S 2$, we describe our targeted SOFIA observations and the observational details of the archival data at each wavelength. In $\S 3$, we describe our aperture-photometry procedures for each data set and discuss our detection rates and trends. We also present sets of multi-scale, multiwavelength images for each object in order to better demonstrate their small- and large-scale properties and overall environments. In $\S 4$. we compare the physical parameters obtained from the various SED modeling methods, including $L / M$, which help to place EGOs into a broader evolutionary context. In $\S 5$ we discuss the implications of our results, and outline future investigations.

\section{THE SAMPLE \& OBSERVATIONS}

In this paper, we conduct a multiwavelength aperturephotometry study of 12 EGOs using the SOFIA Faint Object infraRed CAmera for the SOFIA Telescope (FORCAST Herter et al. 2012). We use new SOFIA FORCAST $19 \mu \mathrm{m}$ and $37 \mu \mathrm{m}$ observations in conjunction with publiclyavailable archival datasets from Spitzer, Herschel, and the Atacama Pathfinder EXperiment 5 (APEX) telescope, to model the SED of the dominant protostar in each of our target EGOs. Details of source properties for our sample are listed in Table 1.

\subsection{SOFIA FORCAST Observations: $19.7 \& 37.1 \mu \mathrm{m}$}

We used SOFIA FORCAST to observe our 12 targets simultaneously at $19.7 \mu \mathrm{m}$ and $37.1 \mu \mathrm{m}$. Observations were performed in the asymmetric chop-and-nod imaging observing mode $\mathrm{C} 2 \mathrm{NC} 2$. The measured ${ }^{6}$ FWHM are $2 . " 5$ at $19.7 \mu \mathrm{m}$ and $3 . " 4$ at $37.1 \mu \mathrm{m}$. At the nearest $(1.13 \mathrm{kpc})$ and farthest $(4.8 \mathrm{kpc})$ source distances, these FWHM correspond to physical size scales of 2,830 to 12,000 au at $19.7 \mu \mathrm{m}$ and 3,840 to 16,300 au at $37.1 \mu \mathrm{m}$. The instantaneous field of view (FOV) of FORCAST is $3.4 \times 3 ! 2$, with pixel size $\theta=0$ !' 768 after distortion correction. This FOV corresponds to $1.1 \times 1.1 \mathrm{pc}$ at a distance of $1.13 \mathrm{kpc}$, and $4.8 \times 4.5 \mathrm{pc}$ at a distance of 4.8 kpc. Table 2 summarizes observation information for each EGO. The project's Plan ID is 04_0159.

Data calibration and reduction are performed by the SOFIA team using the SOFIA data-reduction pipeline 7 . After receipt of the Level 3 data products (artifact-corrected, fluxcalibrated images), we converted our images from Jy pixel ${ }^{-1}$ to $\mathrm{Jy}_{\text {beam }}{ }^{-1}$ in order to more easily perform photometric measurements in CASA (McMullin et al. 2007). Conversion was accomplished by using the CASA task immath to multiply each image by the beam-to-pixel conversion factor $X_{\lambda}=($ beam area $) /($ pixel area $)$. This factor depends on beam size and pixel size, and therefore is different for each wavelength. The beam-to-pixel conversion factors are $\mathrm{X}_{19.7 \mu \mathrm{m}}=$ 12.0067 pixels $/$ beam and $X_{37.1 \mu m}=22.2076$ pixels $/$ beam.

\subsection{Archival Data}

\subsubsection{Spitzer IRAC (GLIMPSE) Observations: $3.6,5.8, \& 8.0 \mu \mathrm{m}$}

All of our EGO targets were originally selected due to their extended emission at $4.5 \mu \mathrm{m}$ as seen in Spitzer GLIMPSE images. In order to constrain the SEDs of the driving sources themselves, we used the archival Spitzer observations at $3.6 \mu \mathrm{m}, 5.8 \mu \mathrm{m}$, and $8.0 \mu \mathrm{m}$ (bands I1, I3, and I4, respectively) from the GLIMPSE project (Benjamin et al. 2003; Churchwell et al. 2009). The point response function (PRF) of the IRAC instrument varies by band and position on the detector. The mean FWHM in bands I1, I3, and I4 are $1 .{ }^{\prime \prime} 66,1 . \prime 72$, and $1 . " 88$, respectively, as detailed in Fazio et al. (2004). All archival GLIMPSE data were downloaded from the NASA/IPAC Infrared Science Archive (IRSA) Gator Cat-

\footnotetext{
5 This publication is based on data acquired with the Atacama Pathfinder Experiment (APEX). APEX is a collaboration between the Max-PlanckInstitut fur Radioastronomie, the European Southern Observatory, and the Onsala Space Observatory.

6 These FWHM are the average values in dual-channel mode for each wavelength as measured by the SOFIA team since Cycle 3. More information can be found in the Cycle 5 Observer's Handbook on the SOFIA website at https://www.sofia.usra.edu/science/proposing-andobserving/sofia-observers-handbook-cycle-5

7 The FORCAST Data Handbook can be found on the SOFIA website at https://www.sofia.usra.edu/science/proposing-and-observing/data-products
} 
TABLE 1

EGO SOURCE PROPERTIES

\begin{tabular}{|c|c|c|c|c|c|c|c|c|}
\hline \multirow[t]{2}{*}{ Source } & \multirow{2}{*}{$\begin{array}{c}V_{\mathrm{LSR}}^{\mathrm{a}} \\
\left(\mathrm{km} \mathrm{s}^{-1}\right)\end{array}$} & \multirow{2}{*}{$\begin{array}{c}\text { Distance }^{\mathrm{b}} \\
\quad(\mathrm{kpc})\end{array}$} & \multirow{2}{*}{$\begin{array}{c}\mathrm{EGO}^{\mathrm{c}} \\
\text { Cat }\end{array}$} & \multirow[t]{2}{*}{ IRDC $^{\mathrm{d}}$} & \multirow{2}{*}{$\begin{array}{l}\mathrm{H}_{2} \mathrm{O}^{\mathrm{e}} \\
\text { Maser }\end{array}$} & \multicolumn{3}{|c|}{$\mathrm{CH}_{3} \mathrm{OH}$ Masers $(\mathrm{GHz})^{\mathrm{f}}$} \\
\hline & & & & & & $6.7^{g}$ & $44^{\mathrm{h}}$ & $95^{\mathrm{i}}$ \\
\hline G10.29-0.13 & 14 & 1.9 & 2 & $\mathrm{Y}$ & $\mathrm{Y}$ & $\mathrm{Y}$ & $\mathrm{Y}$ & $\mathrm{Y}$ \\
\hline G10.34-0.14 & 12 & 1.6 & 2 & $\mathrm{Y}$ & $\mathrm{Y}$ & $\mathrm{Y}$ & $\mathrm{Y}$ & $\mathrm{Y}$ \\
\hline G11.92-0.61 & 36 & $3.38_{-0.27}^{+0.33}(3.5)$ & 1 & $\mathrm{Y}$ & $\mathrm{Y}$ & $\mathrm{Y}$ & $\mathrm{Y}$ & $\mathrm{Y}$ \\
\hline G12.91-0.03 & 57 & 4.5 & 1 & $\mathrm{Y}$ & $\mathrm{Y}$ & $\mathrm{Y}$ & $?$ & $\mathrm{Y}$ \\
\hline G14.33-0.64 & 23 & $1.13_{-0.11}^{+0.14}(2.3)$ & 1 & $\mathrm{Y}$ & $\mathrm{Y}$ & $?$ & $\mathrm{Y}$ & $\mathrm{Y}$ \\
\hline G14.63-0.58 & 19 & $1.83_{-0.07}^{+0.08}(1.9)$ & 1 & $\mathrm{Y}$ & $\mathrm{Y}$ & $\mathrm{Y}$ & $?$ & $\mathrm{Y}$ \\
\hline G16.59-0.05 & 60 & $3.58_{-0.27}^{+0.32}(4.2)$ & 2 & $\mathrm{~N}$ & $\mathrm{Y}$ & $\mathrm{Y}$ & $?$ & $\mathrm{Y}$ \\
\hline G18.89-0.47 & 66 & 4.2 & 1 & $\mathrm{Y}$ & $\mathrm{Y}$ & $\mathrm{Y}$ & $\mathrm{Y}$ & $\mathrm{Y}$ \\
\hline G19.36-0.03 & 27 & 2.2 & 2 & $\mathrm{Y}$ & $\mathrm{N}$ & $\mathrm{Y}$ & $\mathrm{Y}$ & $\mathrm{Y}$ \\
\hline G22.04+0.22 & 51 & 3.4 & 1 & $\mathrm{Y}$ & $\mathrm{Y}$ & $\mathrm{Y}$ & $\mathrm{Y}$ & $\mathrm{Y}$ \\
\hline G28.83-0.25 & 87 & 4.8 & 1 & $\mathrm{Y}$ & $\mathrm{Y}$ & $\mathrm{Y}$ & $\mathrm{Y}$ & $?$ \\
\hline G35.03+0.35 & 53 & $2.32_{-0.20}^{+0.24}(3.2)$ & 1 & $\mathrm{Y}$ & $\mathrm{Y}$ & $\mathrm{Y}$ & $\mathrm{Y}$ & $\mathrm{Y}$ \\
\hline
\end{tabular}

${ }^{a}$ LSRK velocities are the single dish $\mathrm{NH}_{3}(1,1)$ values from Cyganowski et al. (2013).

${ }^{\mathrm{b}}$ Distances without errors are estimated from the LSRK velocity and the Galactic rotation curve parameters from Reid et al. (2014). Parallax distances (with their uncertainties) are given where available from Reid et al. (2014) and references therein, with the kinematic distance in parentheses for comparison. All kinematic distances are the near distance. The uncertainty on each kinematic distance is assumed to be $15 \%$, based on the median percent difference between the parallax-derived and kinematic distances from the five sources which have both.

${ }^{c}$ This is the Table number of the EGO in Cyganowski et al. (2008). In that paper, Tables 1 \& 2 list "likely" EGOs for which 5-band (3.6 to $24 \mu \mathrm{m}$ ) or only $4.5 \mu \mathrm{m}$ Spitzer photometry can be measured, respectively.

${ }^{\mathrm{d}}$ Coincidence of EGO with IRDC as indicated by Cyganowski et al. 2008.

${ }^{\mathrm{e}}$ Water maser data from the Cyganowski et al. (2013) Nobeyama 45-m survey of EGOs.

${ }^{\mathrm{f}}$ Sources for which we could find no information in the literature are indicated by "?".

g The 6.7 GHz maser detection information comes from Cyganowski et al. (2009) using the VLA, except for G12.91-0.03, G14.63-0.58, and G16.59-0.05, which come from Green et al. (2010 and references therein) observations using the Australia Telescope Compact Array (ATCA).

${ }^{\mathrm{h}}$ Information for $44 \mathrm{GHz}$ masers come from the VLA and were taken from Cyganowski et al. (2009), except for G14.33-0.64, which comes from Slysh et al. (1999).

${ }^{\mathrm{i}}$ Most information for $95 \mathrm{GHz}$ masers was taken from Chen et al. (2011) using the Mopra $22 \mathrm{~m}$ telescope. The exceptions are G14.33-0.64 from Val'tts et al. (2000) using Mopra, G16.59-0.05 from Chen et al. (2012) using the Purple Mountain Observatory $13.7 \mathrm{~m}$ telescope, and G35.03+0.35 from Kang et al. (2015) using the Korean VLBA Network.

TABLE 2

SOFIA FORCAST OBSERVING PARAMETERS

\begin{tabular}{ccccccc}
\hline \hline Source & \multicolumn{2}{c}{ Pointing Center (J2000) } & Obs. Date $^{a}$ & \multicolumn{2}{c}{ TOS $^{b}$} & \multicolumn{2}{c}{$\sigma(\mathrm{MAD})^{c}$} \\
& $\mathrm{RA}$ & Dec & & $(\mathrm{s})$ & $37 \mu \mathrm{m}$ & $19 \mu \mathrm{m}$ \\
\hline G10.29-0.13 & $18: 08: 49.2$ & $-20: 05: 59.3$ & 2016 July 13 & 502 & 0.26 & 0.08 \\
G10.34-0.14 & $18: 08: 59.9$ & $-20: 03: 37.3$ & 2016 Sept 27 & 626 & 0.30 & 0.07 \\
G11.92-0.61 & $18: 13: 58.0$ & $-18: 54: 19.3$ & 2016 July 12 & 604 & 0.22 & 0.07 \\
G12.91-0.03 & $18: 13: 48.1$ & $-17: 45: 41.3$ & 2016 July 19 & 1000 & 0.18 & 0.04 \\
G14.33-0.64 & $18: 18: 54.3$ & $-16: 47: 48.3$ & 2016 July 12 & 593 & 0.24 & 0.07 \\
G14.63-0.58 & $18: 19: 15.3$ & $-16: 29: 57.3$ & 2016 July 13 & 641 & 0.22 & 0.07 \\
G16.59-0.05 & $18: 21: 09.0$ & $-14: 31: 50.3$ & 2016 July 20 & 810 & 0.19 & 0.04 \\
G18.89-0.47 & $18: 27: 07.8$ & $-12: 41: 38.3$ & 2016 Sept 27 & 626 & 0.25 & 0.06 \\
G19.36-0.03 & $18: 26: 25.7$ & $-12: 03: 56.3$ & 2016 Sept 20 & 285 & 0.46 & 0.09 \\
G22.04+0.22 & $18: 30: 34.6$ & $-09: 34: 49.3$ & 2016 Sept 20 & 642 & 0.21 & 0.05 \\
G28.83-0.25 & $18: 44: 51.2$ & $-03: 45: 50.3$ & 2016 Sept 27 & 470 & 0.26 & 0.07 \\
G35.03+0.35 & $18: 54: 00.4$ & $+02: 01: 15.7$ & 2016 Sept 22 & 500 & 0.29 & 0.08 \\
\hline
\end{tabular}

${ }^{a}$ All July observations were performed on flights from Christchurch, New Zealand; all September observations were performed on flights from Palmdale, CA, USA.

$\mathrm{b}$ This column lists the total time on source (TOS) for each target. The original proposal called for $600 \mathrm{~s}$ of integration on each source. For four sources, $600 \mathrm{~s}$ could not be achieved due to either high clouds (G19.36) or telescope issues (G10.29, G28.83, G35.03). G12.91 was a shared observation with another group whose observations required additional integration time.

${ }^{c}$ The background noise of the SOFIA images is non-Gaussian in the majority of sources. This column gives the scaled $M A D=1.482 \times M A D$ values for all sources, where $M A D$ is the median absolute deviation from the median background pixel value; $M A D$ must be multiplied by 1.482 to become rmslike. Aperture photometry was performed using cutoffs based on $M A D$ for all sources. $M A D$ values listed here are in $\mathrm{Jy} \mathrm{beam}^{-1}$. 
alog List. The images returned by the archive are all in units of $\mathrm{MJy} \mathrm{sr}^{-1}$.

\subsubsection{Spitzer MIPS (MIPSGAL) Observations: $24 \mu \mathrm{m}$}

We utilized archival $24 \mu \mathrm{m}$ data from the MIPSGAL survey to provide additional mid-IR constraints on our SEDs for 9 of our 12 targets. For the remaining 3 targets (G14.33, G16.59, G35.03), MIPSGAL $24 \mu \mathrm{m}$ data could not be used for the second task due to saturated pixels in the regions of interest. MIPSGAL images have a native brightness unit of $\mathrm{MJy} \mathrm{sr}^{-1}$, and were converted to $\mathrm{Jy}_{\text {beam }}{ }^{-1}$ by first multiplying each image by $1 \times 10^{6}$ (to convert from MJy to Jy) and then multiplying by the solid angle subtended by the 6 ." $0 \times 6$." 0 MIPS beam at $24 \mu \mathrm{m}$. Technical details of the MIPS instrument can be found in Rieke et al. (2004). For details of the MIPSGAL observing program, see Carey et al. (2009) and Gutermuth \& Heyer (2015). All MIPSGAL data were downloaded from the IRSA Gator Catalog List.

\subsubsection{Herschel PACS (Hi-GAL) Observations: $70 \& 160 \mu \mathrm{m}$}

We used archival $70 \mu \mathrm{m}$ and $160 \mu \mathrm{m}$ data from the Herschel Infrared Galactic Plane Survey (Hi-GAL, Molinari et al. 2016), observed with the Herschel Photoconductor Array Camera and Spectrometer (PACS; Poglitsch et al. 2010) instrument, to probe the far-IR portion of the spectrum. These data were originally observed as part of the Herschel HiGAL project (Molinari et al. 2010, 2016) between 2010 October 25 and 2011 November 05 . The observations were performed in parallel mode with a scan speed of $60 \% / \mathrm{s}$. Beam sizes, which are dependent on observing mode, were $\theta_{70 \mathrm{um}}=$ $5.8^{\prime \prime} \times 12.1^{\prime \prime}$ and $\theta_{160 \mu m}=11.4^{\prime \prime} \times 13.4^{\prime \prime}$ as reported in Molinari et al. (2016). The native brightness unit of the Hi-GAL data is $\mathrm{MJy} \mathrm{sr}^{-1}$. Therefore, these images were converted to $\mathrm{Jy}_{\text {beam }}{ }^{-1}$ using the same method as in $\$ 2.2 .2$

We chose to use the Hi-GAL data over the archival PACS data available on the European Space Agency (ESA) Heritage Archive due to the additional astrometric and absolute flux calibration performed by the Hi-GAL team, as detailed in Molinari et al. (2016). All Hi-GAL data were obtained from the Hi-GAL Catalog and Image Server on the Via Lactea web porta 8

\subsubsection{APEX LABOCA (ATLASGAL) Observations: $870 \mu \mathrm{m}$}

We used archival $870 \mu \mathrm{m}$ observations from the APEX Telescope Large Area Survey of the Galaxy (ATLASGAL, Schuller et al. 2009) to populate the submillimeter portion of the SED. The data were retrieved from the ATLASGAL Database Server The ATLASGAL beam size is $19 . " 2$ $\times$ 19.'2; additional observational details can be found in Schuller et al. (2009). These images were already in units of $\mathrm{Jy} \mathrm{beam}^{-1}$, and thus required no unit conversion.

\section{RESULTS}

Figures 1 through 4 show pairs of three-color (RGB) images for each source. The left-hand panels show a 5!3 field of view with 160,70 , and $24 \mu \mathrm{m}$ data mapped to $\mathrm{R}, \mathrm{G}$, and $\mathrm{B}$, respectively, and with $870 \mu \mathrm{m}$ contours overlaid. These panels show the large-scale structure of the cloud and overall environment in which each EGO is located. The righthand panels all have a 1'.0 FOV with the Spitzer IRAC 8.0,

\footnotetext{
${ }^{8} \mathrm{http} / / /$ vialactea.iaps.inaf.it/vialactea/eng/index.php

${ }^{9}$ http://atlasgal.mpifr-bonn.mpg.de/cgi-bin/ATLASGAL_DATABASE.cgi
}

4.5, and $3.6 \mu \mathrm{m}$ data mapped to $\mathrm{R}, \mathrm{G}$, and $\mathrm{B}$, respectively; the extended green emission in these images shows the extent of each EGO. SOFIA FORCAST 19.7 and $37.1 \mu \mathrm{m}$ contours and ATLASGAL $870 \mu \mathrm{m}$ contours are overlaid, and $6.7 \mathrm{GHz}$ $\mathrm{CH}_{3} \mathrm{OH}$ masers (Cyganowski et al. 2009) are marked with diamonds. These panels show the small-scale structure and detailed NIR and MIR emission of each EGO, how this emission relates to the larger-scale $870 \mu \mathrm{m}$ emission, and the locations of any associated markers of MYSOs, such as $6.7 \mathrm{GHz}$ $\mathrm{CH}_{3} \mathrm{OH}$ masers.

Below we discuss in detail the photometric methodology used for each band for the SED analysis. Because the angular resolution and sensitivity - and hence level of confusion vary significantly among the different observations, we have elected to use a photometry method best suited for each particular wavelength in order to minimize (as much as feasible) contamination from unrelated sources. In the following sections we describe in some detail how the photometry was done for each wavelength.

\subsection{SOFIA FORCAST Photometry}

Table 3 shows the photometry for the 19.7 and $37.1 \mu \mathrm{m}$ SOFIA images. In this section, we describe the SOFIA astrometry, source selection, and photometry in more detail.

Astrometry - The SOFIA images required additional astrometric corrections. While the relative astrometry between the 19.7 and $37.1 \mu \mathrm{m}$ data was accurate to less than one pixel, the absolute astrometry of the SOFIA data varied considerably. Relative to the Spitzer MIPS $24 \mu \mathrm{m}$ data, the positions of the SOFIA images varied by up to $\sim 3^{\prime \prime}$. In order to properly register the SOFIA images, we selected field point sources that were present in both the $24 \mu \mathrm{m}$ images and either the 37.1 and $19.7 \mu \mathrm{m}$ images, fit a 2-dimensional gaussian to that point source in both the $24 \mu \mathrm{m}$ and SOFIA frame and applied the calculated position difference to both SOFIA images. In most cases, we were able to find a position match with the $24 \mu \mathrm{m}$ data in only one of the two SOFIA frames, and relied on the sub-pixel relative astrometry between the two SOFIA images in order to correct the non-matched frame. Post-astrometric correction, we consider the absolute astrometric accuracy of the SOFIA images to be dominated by the absolute position uncertainty of the MIPS $24 \mu \mathrm{m}$ images: $\sim 1 . " 4$.

Mid-IR Source Selection and Nomenclature - We limit our analysis to those mid-IR sources we consider to be plausibly associated with the protocluster in which the EGO resides (with some exceptions described below). For short, we call these sources "EGO-associated." In this context, "EGO-associated" means one of two things: a) the mid-IR source is coincident with the extended $4.5 \mu \mathrm{m}$ emission of the EGO and is therefore likely tracing some aspect of the EGO driving source in the mid-IR, or b) the mid-IR source lies outside the $5 \sigma$ level of the $4.5 \mu \mathrm{m}$ emission but is still near to the EGO, and it is unclear whether the source is related or is a field source. In order to create a self-consistent system for selecting sources in the latter category, we establish two criteria: i) the source must lie above the $25 \%$ peak intensity level of the ATLASGAL emission and ii) it must be detected at both 19.7 and $37.1 \mu \mathrm{m}$. If a mid-IR detection is not within the bounds of the $4.5 \mu \mathrm{m}$ emission and does not meet both criteria i) and ii), then it is considered to be a field source.

One source, G14.33-0.64_b meets neither criteria and is likely a field source. It is coincident with the known H II re- 

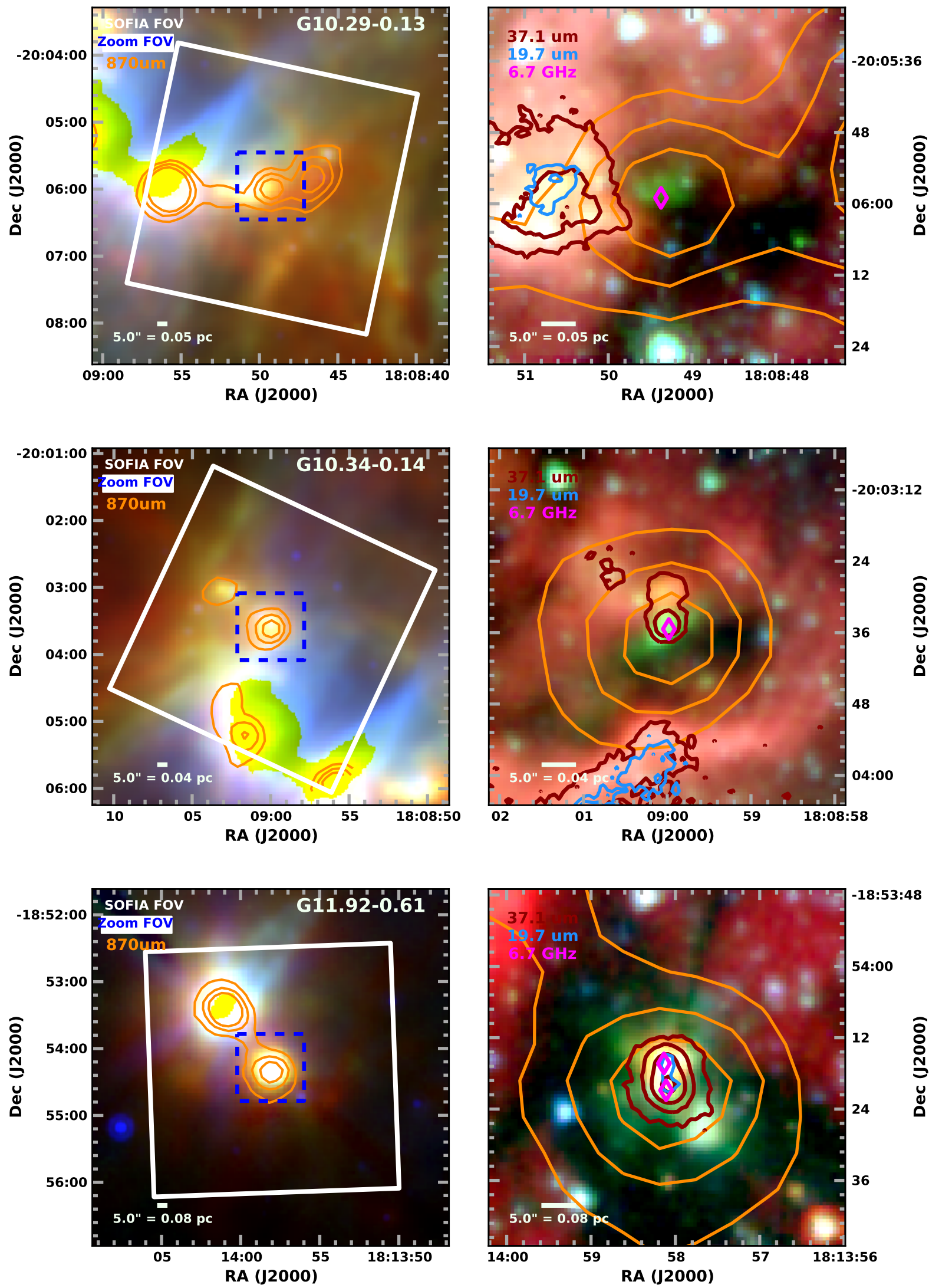

FIG. 1.- RGB images for EGO sources. The left panel for each source shows the $160 \mu \mathrm{m}, 70 \mu \mathrm{m}$, and $24 \mu \mathrm{m}$ wavelengths mapped to R, G, and B, respectively, with $870 \mu \mathrm{m}$ contours overlaid in magenta. The ATLASGAL contour levels are $[0.25,0.5,0.75] \times \mathrm{I}_{\max }$, where $I_{\max }$ is the peak intensity value of the ATLASGAL data in each FOV. The solid white boxes show the size and approximate orientation of the SOFIA FOV, and the dashed blue boxes show the size and position of the FOV of the zoomed images, shown in the right-hand panels. The right-hand panels show the $8.0 \mu \mathrm{m}, 4.5 \mu \mathrm{m}$, and $3.6 \mu \mathrm{m}$ wavelengths mapped to R, G, and B, respectively; the extended green emission shows the extent of each EGO. SOFIA FORCAST $19.7 \mu \mathrm{m}$ and $37.1 \mu \mathrm{m}$ contours are overlaid in blue and red, respectively, and the positions of known $6.7 \mathrm{GHz}$ Class II $\mathrm{CH}_{3} \mathrm{OH}$ masers are denoted by magenta diamonds. SOFIA contour levels are [5,15,45,125,250] $\times \sigma$ for $37.1 \mu \mathrm{m}$, and $[4,8,16,28] \times \sigma$ for $19.7 \mu \mathrm{m}$, where $\sigma$ is the scaled MAD. The $I_{\max }$ values for G10.29-0.13, G10.34-0.14, and G11.92-0.61 are 3.31 Jy beam ${ }^{-1}$, $4.39 \mathrm{Jy} \mathrm{beam}^{-1}$, and $4.01 \mathrm{Jy} \mathrm{beam}^{-1}$, respectively. 

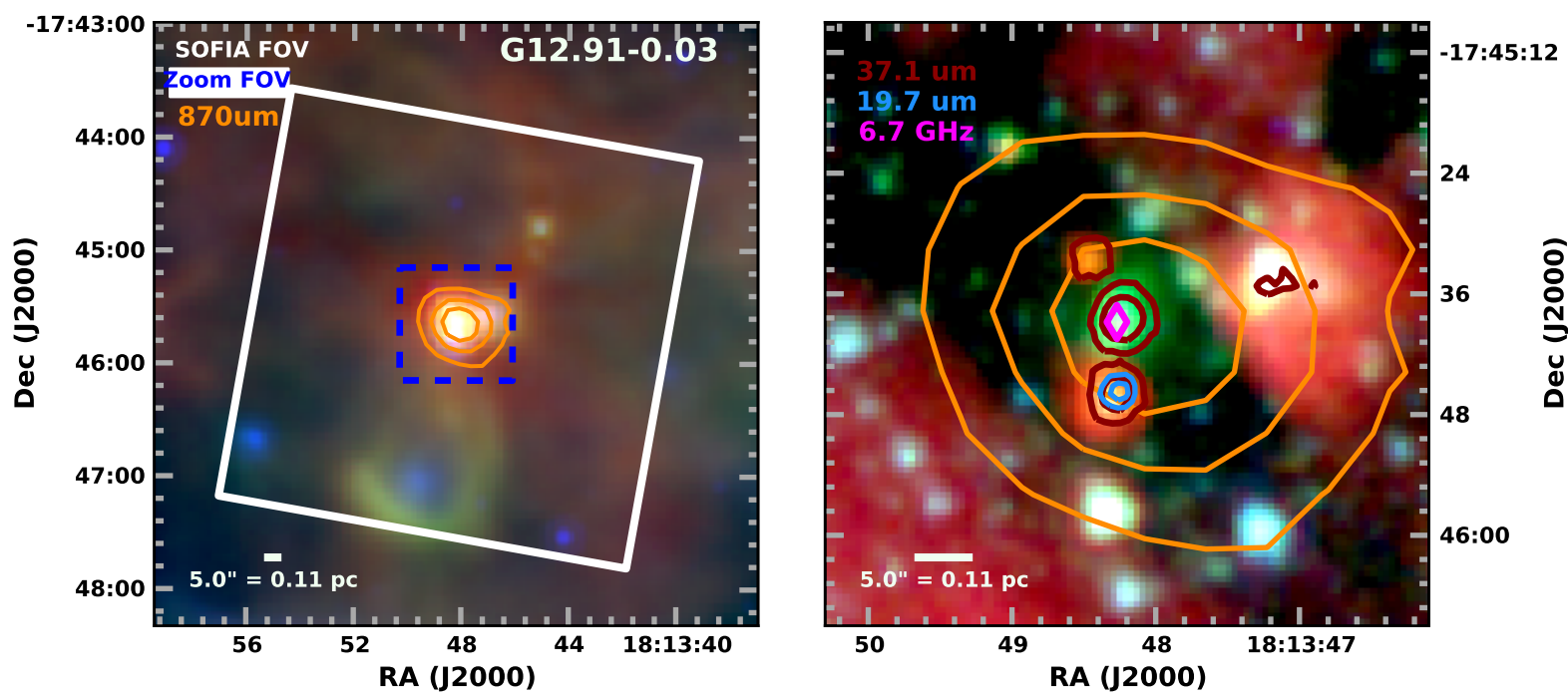

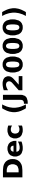
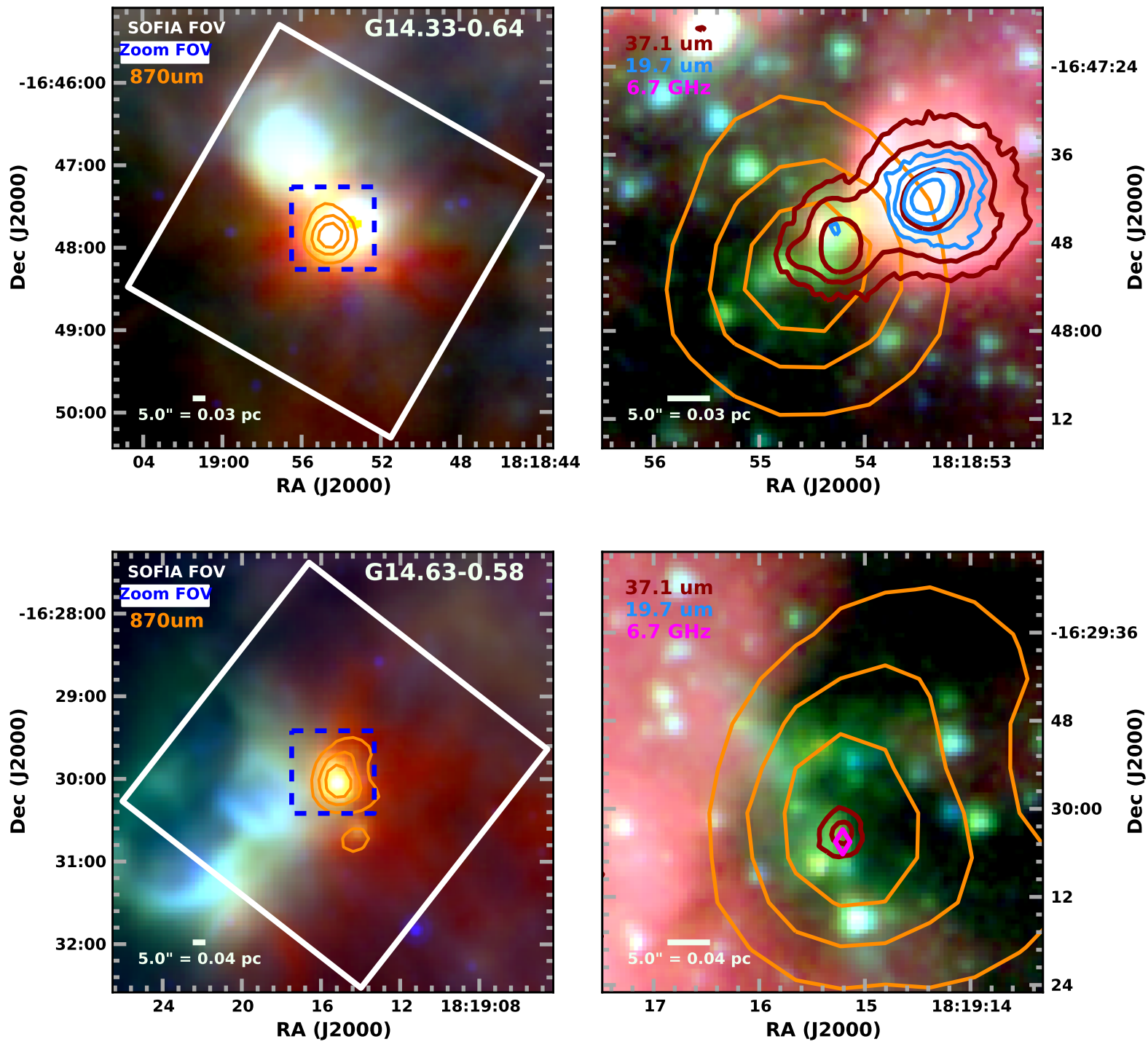

FIG. 2.- RGB images for EGO sources. See Figure 1 for key. The $I_{\max }$ values for G12.91-0.03, G14.33-0.64, and G14.63-0.58 are 2.78 Jy beam ${ }^{-1}, 12.98$ $\mathrm{Jy} \mathrm{beam}^{-1}$, and $4.35 \mathrm{Jy} \mathrm{beam}^{-1}$, respectively. 

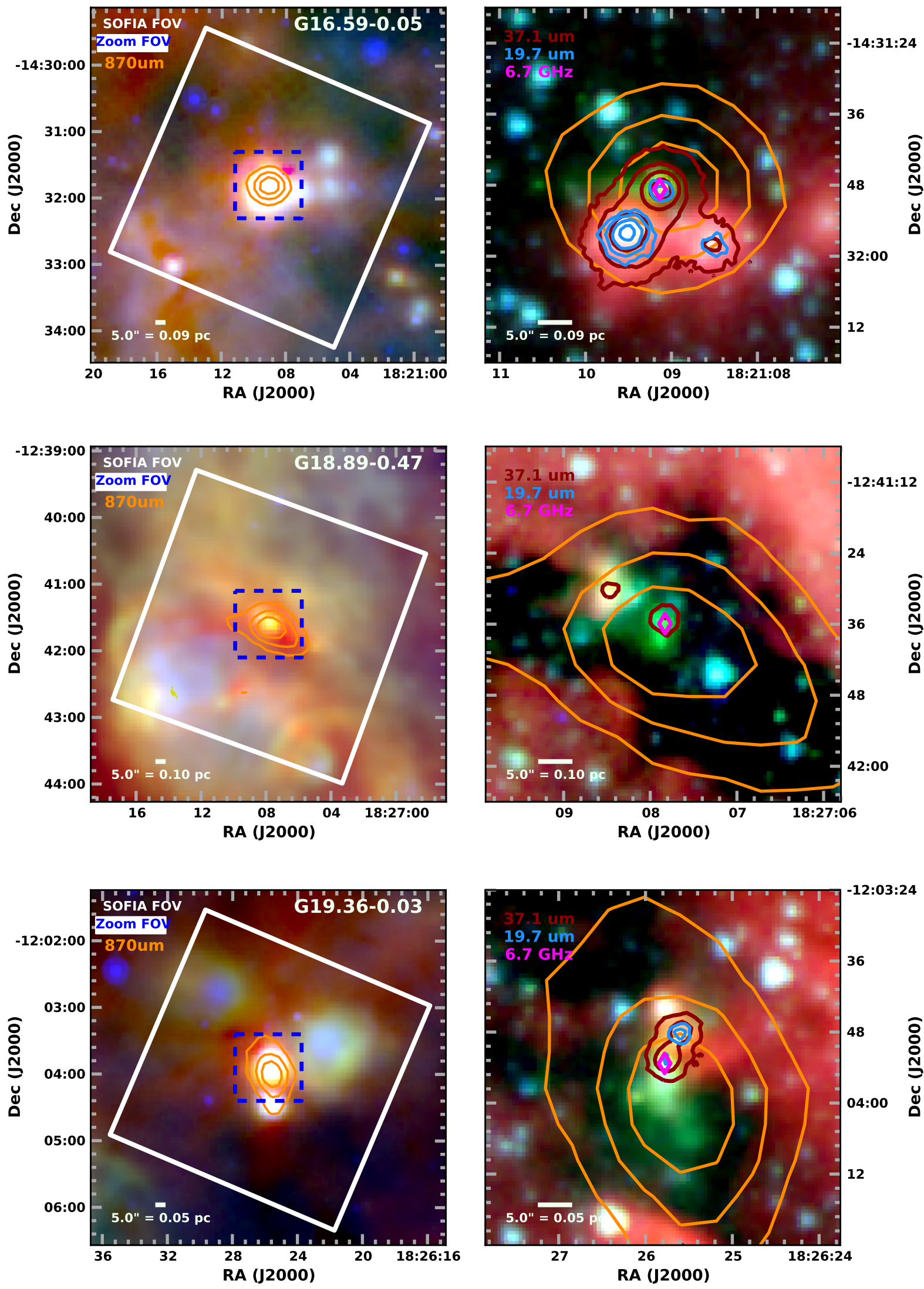

FIG. 3.- RGB images for EGO sources. See Figure 1 for key. The $I_{\max }$ values for G16.59-0.05, G18.89-0.47, and G19.36-0.03 are 5.13 Jy beam ${ }^{-1}, 3.30$ $\mathrm{Jy} \mathrm{beam}^{-1}$, and $2.90 \mathrm{Jy}$ beam $^{-1}$, respectively. 

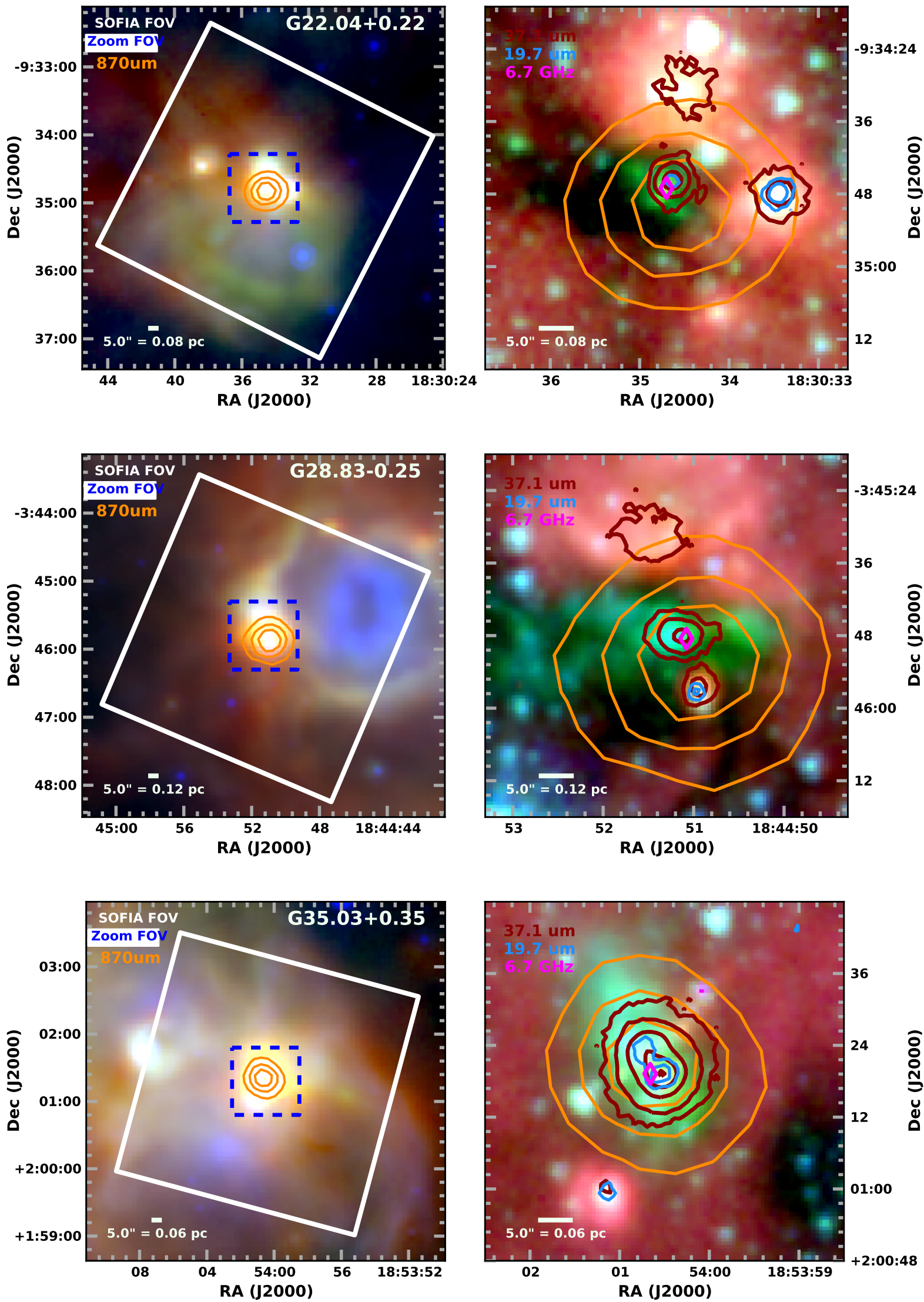

FIG. 4.- RGB images for EGO sources. See Figure 1 for key. The $I_{\max }$ values for G22.04+0.22, G28.83-0.25, and G35.03+0.35 are 3.33 Jy beam ${ }^{-1}, 4.08$ $\mathrm{Jy} \mathrm{beam}^{-1}$, and $4.91 \mathrm{Jy}^{\text {beam }}{ }^{-1}$, respectively. 
gion IRAS 18159-1648. However, it was necessary to explicitly fit this source in order to get accurate flux density results for the EGO-associated sources.

For a given EGO field, source "a" is always the brightest EGO-associated source at $37 \mu \mathrm{m}$, source "b" is the secondbrightest at $37 \mu \mathrm{m}$ (of all analyzed sources for that FOV), and so on in order of decreasing brightness. The $37 \mu \mathrm{m}$ source name designations are used for all the wavelengths analyzed in this paper.

Photometry - After source selection, we fit each source with 2-dimensional gaussian functions using the CASA task imfit in order to determine the total flux density, peak intensity, and major and minor axes. We then applied a multiplicative correction factor (an "aperture correction") to each fitted flux in order to account for the deviation of the SOFIA PSF from a true gaussian. Our detailed procedure was as described below.

We first selected emission-free regions in each image in order to determine the background noise levels. These emission-free regions are identical for all three mid-IR data sets (SOFIA $37.1 \mu \mathrm{m}$ and $19.7 \mu \mathrm{m}$, and MIPS $24 \mu \mathrm{m}$ ) for a given source. However, the SOFIA images in particular have background levels that typically do not show noise variations about zero. Therefore, we chose to use the scaled MAD as an estimate of the noise $(1.482 \times \mathrm{MAD}$, where MAD is the median absolute deviation from the median), rather than the rms or standard deviation. With the exception of the ATLASGAL data, all data sets analyzed in this work have noise variations that are not centered about zero. Therefore, we have used the scaled MAD for all data sets for the sake of consistency. From this point forward, the " $\sigma$ " symbol refers to the scaled MAD whenever we are estimating or discussing background noise levels of the images.

We then performed the fitting for each source using imfit. We iteratively refined each fit (e.g. by holding certain parameters, such as source position, fixed during the fit) until we determined the fit to be satisfactory. We declared a fit to be satisfactory once the absolute value of the residual intensities of all pixels in the central Airy disk were below $4 \times \mathrm{MAD}$ of the residual image, with the majority below $2 \times$ MAD. In cases where source parameters are held fixed, imfit does not return an uncertainty for those specific parameters, so the uncertainty is due entirely due to user choice of source position, size, etc. For these fits, our position uncertainties are 0.01 pixels, and uncertainties in the major and minor axes or position angles are $0.1^{\circ}$. All the uncertainties for parameters held fixed during the fit are listed in italics in Table 3

Finally, we determined a wavelength-dependent multiplicative correction factor to the imfit flux results. The SOFIA PSF is an obscured Airy diffraction pattern - its central bright disk has a slightly narrower width than a standard Airy diffraction pattern due to the effect of a central obscuration in the light path (the secondary mirror). However, imfit only fits 2-dimensional Gaussians. In effect, it fits a Gaussian to the central Airy disk and ignores the surrounding Airy rings. These correction factors are effectively serving as "aperture corrections" for our data; the only difference is that they are corrections to the fitted flux values returned by imfit, rather than corrections to direct measurements. As Airy diffraction patterns are wavelength-dependent, we calculated separate aperture corrections for our $19.7 \mu \mathrm{m}$ and $37.1 \mu \mathrm{m}$ data. While the best practice in aperture photometry would be to measure the PSF of an unrelated, isolated point source in each field and then apply that PSF correction to the data, we found that almost none of our fields contained an unrelated point source, much less one bright enough to measure the PSF with any confidence. Instead, we employed the procedure described below.

We first created four $100 \times 100$-pixel Airy diffraction patterns using the optical properties of the SOFIA telescope (primary and secondary mirror size and separation, etc.) at each of our two wavelengths. The PSFs are sampled with $0 .{ }^{\prime \prime} 768$ pixels, the same as the FORCAST instrument. At this pixel size, the total grid is $76 . " 8$ in diameter; this is $\sim 23$ times the FWHM at $37.1 \mu \mathrm{m}$ (3." 4 ) as quoted in the Handbook, and $\sim 31$ times the FWHM at $19.7 \mu \mathrm{m}\left(2{ }^{\prime \prime} 5\right)$. Although Airydisk diffraction patterns mathematically extend to infinity, on a practical level, our synthetic PSFs had to be truncated to a particular size; we considered $>20$ times the quoted FWHM to be sufficient. The four PSFs for a given wavelength are mathematically identical, but each center position is given either zero- or half-pixel offsets in both the $\mathrm{x}$ and $\mathrm{y}$ directions. This effectively gives us four different sampling scenarios for the PSF. This was done to account for the fact that the peak of a given point source might not always fall neatly onto a single pixel, but instead might be sampled relatively equally between two or even four pixels. We then used imfit to fit the central disk of each of these four PSFs, and compared the flux returned by imfit for the central disk alone to the flux measured within an aperture of radius 50 pixels (38." 4 at $0 . \prime 768$ per pixel; 50 pixels was the largest aperture radius available to us for a $100 \times 100$-pixel grid). We calculated the ratio of measured to fitted fluxes for each of the four PSF grids for one wavelength, and took the mean of these ratios as our aperture correction factor for that wavelength. The aperture correction at $37 \mu \mathrm{m}$ is $1.17 \pm 0.02$, and the aperture correction at $19 \mu \mathrm{m}$ is $1.11 \pm 0.04$, where the uncertainties are the standard deviation of the four measured-to-fitted flux ratios at each wavelength.

Table 3 shows the aperture-corrected imfit results for our $37 \mu \mathrm{m}$ and $19 \mu \mathrm{m}$ data. Non-detections are noted as upper limits. Our detection rate at $37 \mu \mathrm{m}$ is $92 \%$; the only target for which we did not detect any $37 \mu \mathrm{m}$ emission is G10.290.13 . Overall, we detect 24 separate $37 \mu \mathrm{m}$ sources in our 12 targets. Our detection rate at $19 \mu \mathrm{m}$ is slightly lower we detect $19 \mu \mathrm{m}$ emission in only 9 of our 12 targets, for a detection rate of $75 \%$. Overall, we detect 18 separate $19 \mu \mathrm{m}$ sources in our 12 targets.

The uncertainties on the integrated flux density values are the quadrature sum of three values: the fitted-flux uncertainties returned by the imfit task, the uncertainty of our measured aperture corrections, and the absolute flux calibration uncertainty for the SOFIA FORCAST data. Herter et al. (2012) quote an absolute flux calibration accuracy to within $20 \%$ of the total integrated flux for a given object, and that is the value we adopt here. The uncertainties on the integrated flux densities returned by imfit are set by the background noise level, which we set to the scaled MAD for each source and wavelength during the fitting procedure. The uncertainties of our aperture correction factors are discussed above.

\subsection{Photometry of Archival Data}

\subsubsection{Spitzer IRAC Photometry}

In order to constrain the near-infrared portion of the SEDs, we chose to perform aperture photometry for our targets at $3.6 \mu \mathrm{m}, 5.8 \mu \mathrm{m}$, and $8.0 \mu \mathrm{m}$ (the IRAC I1, I3, and I4 bands, 
TABLE 3

SOFIA FORCAST $19.7 \mu \mathrm{M}$ AND $37.1 \mu \mathrm{M}$ FITTED FLuX DENSITIES

\begin{tabular}{|c|c|c|c|c|c|c|c|}
\hline \multirow[t]{2}{*}{ EGO } & \multirow[t]{2}{*}{ Source $^{a}$} & \multicolumn{2}{|c|}{ Coordinates $(\mathrm{J} 2000)^{b}$} & \multicolumn{2}{|l|}{ Fitted Size ${ }^{b}$} & \multirow{2}{*}{$\begin{array}{l}19 \mu \mathrm{m} \text { Flux }^{c} \\
\text { Density (Jy) }\end{array}$} & \multirow{2}{*}{$\begin{array}{l}37 \mu \mathrm{m} \text { Flux } \\
\text { Density (Jy) }\end{array}$} \\
\hline & & RA (h m s) & $\operatorname{Dec}(0 / \prime \prime)$ & Major $\times$ Minor $\left({ }^{\prime \prime} \times{ }^{\prime \prime}\right)$ & $\mathrm{PA}\left({ }^{\circ}\right)$ & & \\
\hline G10.29-0.13 & $\mathrm{a}$ & & & & & $<0.40$ & $<1.3$ \\
\hline \multirow[t]{2}{*}{ G10.34-0.14 } & $\mathrm{a}$ & 18:09:00.001 (0.003) & $-20: 03: 34.53(0.04)$ & $1.41 \times 1.01(0.31 \times 0.45)$ & $97(31)$ & $<0.36$ & $14(3)$ \\
\hline & $\mathrm{b}$ & 18:09:00.02 (0.02) & $-20: 03: 28.8(0.2)$ & $4.55 \times 2.97(0.71 \times 0.61)$ & $97(15)$ & $0.7(0.2)$ & $9(2)$ \\
\hline \multirow[t]{2}{*}{ G11.92-0.61 } & $\mathrm{a}$ & $18: 13: 58.078(0.002)$ & $-18: 54: 20.16(0.04)$ & $2.63 \times 2.39(0.1 \times 0.05)$ & $32(9)$ & $1.1(0.4)$ & $57(12)$ \\
\hline & $\mathrm{b}$ & $18: 13: 58.113(0.008)$ & $-18: 54: 16.25(0.09)$ & $3.41 \times 2.59(0.14 \times 0.13)$ & $100(6)$ & $0.7(0.3)$ & $25(5)$ \\
\hline \multirow[t]{2}{*}{ G12.91-0.03 } & $\mathrm{a}$ & $18: 13: 48.227(0.004)$ & $-17: 45: 38.46(0.06)$ & $2.17 \times 1.58(0.26 \times 0.30)$ & $148(17)$ & $0.2(0.1)$ & $9(2)$ \\
\hline & $\mathrm{c}$ & $18: 13: 48.44(0.01)$ & $-17: 45: 32.5(0.2)$ & $1.43 \times 1.11(0.84 \times 0.91)$ & $35(140)$ & $<0.20$ & $2.2(0.6)$ \\
\hline \multirow[t]{3}{*}{ G14.33-0.64 } & $\mathrm{a}$ & $18: 18: 54.232(0.003)$ & $-16: 47: 48.40(0.06)$ & $5.40 \times 3.41(0.08 \times 0.06)$ & $4.2(1.3)$ & $0.7(0.3)$ & $85(17)$ \\
\hline & $\mathrm{b}$ & 18:18:53.36 (0.01) & $-16: 47: 42.3(0.1)$ & $11.42 \times 10.23(0.15 \times 0.13)$ & $107(5)$ & $22(5)$ & $200(41)$ \\
\hline & $\mathrm{c}$ & 18:18:54.64 (0.01) & $-16: 47: 49.6(0.1)$ & point source & pt. src. & $<0.33$ & $7(1)$ \\
\hline G14.63-0.58 & $\mathrm{a}$ & $18: 19: 15.225(0.009)$ & $-16: 30: 03.3(0.1)$ & $3.60 \times 2.91(0.41 \times 0.39)$ & $59(22)$ & $<0.34$ & $10(2)$ \\
\hline \multirow[t]{3}{*}{ G16.59-0.05 } & $\mathrm{a}$ & $18: 21: 09.124(0.002)$ & $-14: 31: 48.79(0.03)$ & $2.47 \times 2.41(0.04 \times 0.04)$ & 89 (29) & $0.7(0.2)$ & $67(14)$ \\
\hline & $\mathrm{b}$ & $18: 21: 09.509(0.001)$ & $-14: 31: 55.88(0.01)$ & $6.14 \times 5.18(0.1 \times 0.09)$ & $141(4)$ & $5(1)$ & $68(14)$ \\
\hline & $\mathrm{c}$ & $18: 21: 08.511(0.001)$ & $-14: 31: 57.92(0.01)$ & $1.91 \times 1.91(0.1 \times 0.1)$ & $167(360)$ & $0.8(0.3)$ & $6(1)$ \\
\hline G18.89-0.47 & $\mathrm{a}$ & $18: 27: 07.835(0.008)$ & $-12: 41: 35.1(0.1)$ & $1.93 \times 1.85(0.70 \times 0.72)$ & $167(307)$ & $<0.31$ & $5(1)$ \\
\hline \multirow[t]{2}{*}{ G19.36-0.03 } & $\mathrm{a}$ & $18: 26: 25.750(0.005)$ & $-12: 03: 52.57(0.07)$ & $2.69 \times 2.48(0.28 \times 0.28)$ & $70(52)$ & $0.3(0.1)$ & $24(5)$ \\
\hline & $\mathrm{b}$ & $18: 26: 25.591(0.009)$ & $-12: 03: 47.9(0.1)$ & $3.78 \times 2.56(0.41 \times 0.39)$ & $84(12)$ & $1.7(0.5)$ & $20(4)$ \\
\hline \multirow[t]{2}{*}{ G22.04+0.22 } & $\mathrm{a}$ & $18: 30: 34.635(0.003)$ & $-09: 34: 45.74(0.04)$ & $2.13 \times 1.57(0.13 \times 0.15)$ & $135(9)$ & $0.4(0.2)$ & $21(4)$ \\
\hline & $\mathrm{b}$ & $18: 30: 33.43(0.01)$ & $-09: 34: 47.9(0.2)$ & $4.81 \times 4.34(0.40 \times 0.38)$ & $95(32)$ & $1.8(0.4)$ & $14(3)$ \\
\hline \multirow[t]{2}{*}{ G28.83-0.25 } & $\mathrm{a}$ & $18: 44: 51.138(0.001)$ & $-03: 45: 48.05(0.01)$ & $4.41 \times 2.53(0.16 \times 0.14)$ & $87.5(0.1)$ & $0.3(0.2)$ & $32(7)$ \\
\hline & $\mathrm{b}$ & $18: 44: 50.938(0.008)$ & $-03: 45: 56.5(0.2)$ & $2.61 \times 1.34(0.42 \times 0.63)$ & $159(12)$ & $1.1(0.3)$ & $8(2)$ \\
\hline \multirow[t]{2}{*}{$\mathrm{G} 35.03+0.35$} & $\mathrm{a}$ & $18: 54: 00.524(0.003)$ & $+02: 01: 19.16(0.04)$ & $3.64 \times 3.10(0.03 \times 0.03)$ & $115(2)$ & $1.5(0.4)$ & $160(31)$ \\
\hline & $\mathrm{b}$ & $18: 54: 00.700(0.007)$ & +02:01:23.2(0.1) & $5.47 \times 3.91(0.09 \times 0.07)$ & $131(2)$ & $0.8(0.3)$ & $100(20)$ \\
\hline
\end{tabular}

a The listed source positions and fitted sizes are from the $37.1 \mu \mathrm{m}$ fit results only.

b G10.29-0.13 has " ..." in place of coordinates and fitted size because it was a non-detection at both wavelengths.

${ }^{\mathrm{c}}$ Upper limits are given for sources that have no emission above $5 \sigma$. In these cases, the listed upper limit is the $5 \sigma$ value for the FOV.

respectively) using CASAViewer. However, because the flux in these bands likely includes emission from some sources or processes unrelated to our sources of interest, and because the SED models we employ in $\$ 4.2$ do not include emission from PAHs, we chose to include these data as upper limits.

We obtained the necessary IRAC images from the NASA/IPAC Gator Catalog List, and aperture corrections were applied to each measurement according to the table on page 27 of the IRAC Instrument Handbook 10 We did not include measurements in the $4.5 \mu \mathrm{m}$ (I2) band because the emission in this band is extended in all cases (this was the original classification criterion for this object type).

The background noise level for the IRAC bands, as for all other wavelengths, is the scaled MAD within an emissionfree region in each image. The emission-free regions were identical for all three IRAC bands used. For each source with significant $(>5 \sigma)$ emission at $37.1 \mu \mathrm{m}$, we measured the integrated IRAC band flux within a circular aperture centered on the $37.1 \mu \mathrm{m}$ coordinates. We also measured the flux within an annulus of corresponding size. Aperture and annulus sizes were chosen based on the aperture corrections listed in the IRAC Instrument Handbook and the FWHM of each source in each band. For a given source, we used the same aperture for all three IRAC bands (i.e. we did not modify the size of the aperture with wavelength); we chose the smallest aperture that would successfully fit a source in all three bands. Each integrated flux measurement was corrected for background emission by subtracting the product of the median intensity value within the annulus and the size of the aperture from the direct aperture-flux measurement. After this subtraction, we applied the appropriate aperture corrections as listed in the IRAC Instrument Handbook. All aperture and annulus radii, aperture corrections, and corrected fluxes for our sources are listed in Table 4

Due to the very crowded nature of these fields in the IRAC bands and the generally clustered nature of our sources, it was sometimes necessary to use annuli for local background subraction that were not centered on our sources. When this was necessary, we chose isolated stars within the same field of view and centered our annuli on those sources. We were careful to choose annulus stars of similar or lower brightness than the source in question. Choosing a star of equal or lower brightness for background subtraction would only have the effect of increasing the measured flux density. While it does sacrifice some precision, allowing the measured flux density to perhaps be artificially increased maintains the selfconsistency of the photometry, as the data from these bands will only be used as upper limits.

The uncertainties on the integrated flux densities are the quadrature sum of three values: the background noise levels, the absolute flux calibration uncertainty for the IRAC bands, and the uncertainty in the aperture-correction values. The background noise levels are discussed above. The IRAC Instrument Handbook quotes an absolute flux calibration accuracy to within $3 \%$ of the total integrated flux for a given object, and that is the value we adopt here. Additionally, the Handbook quotes an absolute aperture-correction accuracy to within $2 \%$ of the total aperture-correction factor.

\subsubsection{Spitzer MIPS Photometry}

We used CASA's imfit task to determine the integrated flux densities of our targets at $24 \mu \mathrm{m}$ using the same fitting procedure described in $\S 3.1$. Due to the MIPS $24 \mu \mathrm{m}$ images' significant Airy rings for point sources (up to $22 \%$ of the

\footnotetext{
${ }^{10} \mathrm{https} / /$ irsa.ipac.caltech.edu/data/SPITZER/docs/irac/iracinstrumenthandbook/total integrated flux according to the MIPS Instrument Hand-
} 
TABLE 4

IRAC INTEGRATED FLUX DENSITIES

\begin{tabular}{|c|c|c|c|c|c|c|c|c|c|}
\hline \multirow[t]{2}{*}{ EGO } & \multirow[t]{2}{*}{ Source } & \multirow{2}{*}{$\begin{array}{l}\text { Aperture } a \\
\text { Radius }\left({ }^{\prime \prime}\right)\end{array}$} & \multirow{2}{*}{$\begin{array}{l}\text { Annulus }^{a} \\
\left.\text { Radii (" }{ }^{\prime \prime}\right)\end{array}$} & \multicolumn{3}{|c|}{ Aperture Correction $b$} & \multicolumn{3}{|c|}{ Aperture-Corrected Flux Density (mJy) } \\
\hline & & & & $3.6 \mu \mathrm{m}$ & $5.8 \mu \mathrm{m}$ & $8.0 \mu \mathrm{m}$ & $3.6 \mu \mathrm{m}$ & $5.8 \mu \mathrm{m}$ & $8.0 \mu \mathrm{m}$ \\
\hline G10.29-0.13 & a & 3.6 & 3.6 to 8.4 & 1.125 & 1.135 & 1.221 & $0.4(0.3)$ & $15(3)$ & $22(8)$ \\
\hline \multirow{2}{*}{ G10.34-0.14 } & $\mathrm{a}$ & 3.6 & 3.6 to 8.4 & 1.125 & 1.135 & 1.221 & $16.0(0.8)$ & $94(6)$ & 99 (14) \\
\hline & $\mathrm{b}$ & 3.6 & 3.6 to 8.4 & 1.125 & 1.135 & 1.221 & $11.2(0.6)$ & $137(7)$ & $350(21)$ \\
\hline \multirow[t]{2}{*}{ G11.92-0.61 } & $\mathrm{a}$ & 2.4 & 2.4 to 7.2 & 1.215 & 1.366 & 1.568 & $10.0(0.4)$ & $66(3)$ & $40(2)$ \\
\hline & $\mathrm{b}$ & 2.4 & 2.4 to 7.2 & 1.215 & 1.366 & 1.568 & $22.9(0.9)$ & $193(7)$ & $152(6)$ \\
\hline \multirow[t]{3}{*}{ G12.91-0.03 } & a & 3.6 & 3.6 to 8.4 & 1.125 & 1.135 & 1.221 & $8.7(0.4)$ & $52(3)$ & $20(5)$ \\
\hline & b & 4.8 & 14.4 to 24.0 & 1.070 & 1.076 & 1.087 & $1.3(0.2)$ & $55(4)$ & $130(13)$ \\
\hline & $\mathrm{c}$ & 3.6 & 3.6 to 8.4 & 1.125 & 1.135 & 1.221 & $0.5(0.2)$ & $15(2)$ & $34(5)$ \\
\hline \multirow[t]{3}{*}{ G14.33-0.64 } & $\mathrm{a}$ & 4.8 & 14.4 to 24.0 & 1.070 & 1.076 & 1.087 & $6.6(0.4)$ & $86(4)$ & $136(9)$ \\
\hline & $\mathrm{b}$ & 12.0 & 14.4 to 24.0 & 1.000 & 1.000 & 1.000 & $141(6)$ & $1600(70)$ & $4300(180)$ \\
\hline & $\mathrm{c}$ & 3.6 & 3.6 to 8.4 & 1.125 & 1.135 & 1.221 & $2.1(0.2)$ & $26(1)$ & $31(3)$ \\
\hline G14.63-0.58 & $\mathrm{a}$ & 2.4 & 2.4 to 7.2 & 1.215 & 1.366 & 1.568 & $0.9(0.1)$ & $10.5(0.1)$ & $13(2)$ \\
\hline \multirow[t]{3}{*}{ G16.59-0.05 } & $\mathrm{a}$ & 3.6 & 3.6 to 8.4 & 1.125 & 1.135 & 1.221 & $3.3(0.3)$ & $108(5)$ & $107(7)$ \\
\hline & $\mathrm{b}$ & 6.0 & 6.0 to 12.0 & 1.060 & 1.063 & 1.084 & $60(3)$ & $740(30)$ & $1840(80)$ \\
\hline & $\mathrm{c}$ & 6.0 & 6.0 to 12.0 & 1.060 & 1.063 & 1.084 & $43(2)$ & 330 (15) & $820(40)$ \\
\hline \multirow[t]{2}{*}{ G18.89-0.47 } & $\mathrm{a}$ & 4.8 & 14.4 to 24.0 & 1.070 & 1.076 & 1.087 & $4.6(0.6)$ & $46.2(0.4)$ & $20(11)$ \\
\hline & $\mathrm{b}$ & 4.8 & 14.4 to 24.0 & 1.070 & 1.076 & 1.087 & $22(1)$ & $104(6)$ & $200(15)$ \\
\hline \multirow[t]{2}{*}{ G19.36-0.03 } & a & 4.8 & 14.4 to 24.0 & 1.070 & 1.076 & 1.087 & $24(1)$ & $151(7)$ & $190(12)$ \\
\hline & b & 3.6 & 3.6 to 8.4 & 1.125 & 1.135 & 1.221 & $11.0(0.5)$ & $101(4)$ & $220(10)$ \\
\hline \multirow[t]{2}{*}{ G22.04+0.22 } & $\mathrm{a}$ & 4.8 & 14.4 to 24.0 & 1.070 & 1.076 & 1.087 & $1.4(0.3)$ & $30(2)$ & $26(6)$ \\
\hline & $\mathrm{b}$ & 9.6 & 14.4 to 24.0 & 1.011 & 1.011 & 1.017 & $79(4)$ & 791 (32) & $1980(83)$ \\
\hline \multirow[t]{2}{*}{ G28.83-0.25 } & a & 4.8 & 14.4 to 24.0 & 1.070 & 1.076 & 1.087 & $23(1)$ & $77(5)$ & $17(8)$ \\
\hline & $\mathrm{b}$ & 4.8 & 14.4 to 24.0 & 1.070 & 1.076 & 1.087 & $8.0(0.6)$ & $91(5)$ & $220(13)$ \\
\hline \multirow[t]{2}{*}{ G35.03+0.35 } & a & 3.6 & 3.6 to 8.4 & 1.125 & 1.135 & 1.221 & $17.0(0.8)$ & $140(6)$ & $58(6)$ \\
\hline & $\mathrm{b}$ & 3.6 & 3.6 to 8.4 & 1.125 & 1.135 & 1.221 & $30(1)$ & $121(6)$ & $34(6)$ \\
\hline
\end{tabular}

${ }^{a}$ These columns list the radii of the aperture and annuli used for aperture photometry for each source. Radii are listed in arcseconds. Pixel scale is 1. ' 2 /pixel for all IRAC bands.

b Aperture correction factors are from the IRAC Instrument Handbook.

book ${ }^{11}$, the MIPS fit results require an aperture correction similar to that discussed in $\S 3.1$. Fortunately, the MIPS images, unlike our SOFIA images, contain a plethora of isolated point sources with which to measure the PSF directly.

In order to determine the value of the necessary aperture correction, we performed the imfit fitting procedure described in $\$ 3.1$ on five isolated, relatively bright point sources with fluxes listed in the MIPSGAL Point Source Catalog (Gutermuth \& Heyer 2015). We selected the sources to span a range of colors and $24 \mu \mathrm{m}$ flux densities. As with the SOFIA sources, we considered fits to be "satisfactory" when the absolute value of the residuals within the Airy disk were all under $4 \times$ MAD of the residual image, with the majority under $2 \times$ MAD. We compared the integrated fluxes returned by the imfit task to those listed in the MIPSGAL Point Source Catalog. We found a consistent aperture correction value of $1.59 \pm 0.00893$. Table 5 shows the positions, catalog fluxes, fitted flux results, and calculated flux ratios for these five standard stars.

We then applied the fitting procedure and measured aperture correction to our science targets. As for the SOFIA FORCAST data, sometimes certain parameters (source position, size, etc.) were held fixed during the fitting procedure; these cases are noted in Table 6 . Our results are listed in Table 6, which presents the final, aperture-corrected fitted flux results (to be used in the SED fitting) as well as the initial, un-corrected imfit flux results.

As with the SOFIA data, the uncertainties on the integrated flux density values are the quadrature sum of the uncertainties returned by the imfit task, the uncertainty of our calculated aperture-correction value, and the absolute flux calibration uncertainty for the MIPS data. The MIPS Instrument Hand-

\footnotetext{
${ }^{11} \mathrm{http} / / /$ irsa.ipac.caltech.edu/data/SPITZER/docs/mips/mipsinstrumenthandbook٪able 7
}

book quotes an absolute flux calibration accuracy to within $5 \%$ of the total integrated flux of a given object. The uncertainties on both the peak intensity and the integrated flux density returned by imfit are set by the background noise level, which we set to the scaled MAD during the fitting procedure. The uncertainty of the calculated aperture correction value we take to be the standard deviation of the five measured values: $8.93 \times 10^{-3}$.

\subsubsection{Hi-GAL and ATLASGAL Photometry}

Unlike with the near- and mid-infrared data sets, our farIR data could rarely be considered point-like. Therefore, instead of fitting gaussians to the emission using imfit, we measured the integrated flux of each source within a given intensity level using CASAViewer. The intensity levels were chosen uniquely for each source depending on local background emission and the overall image noise level $(\sigma)$. Generally, apertures for the ATLASGAL data followed the $5 \sigma$ level. Apertures for the Hi-GAL data varied between $60 \sigma$ and $200 \sigma$ at $70 \mu \mathrm{m}$ and between $40 \sigma$ and $150 \sigma$ at $160 \mu \mathrm{m}$. These apertures follow comparatively high contours due to the combination of low scaled $M A D$ values (typically of order $10^{-1}$ ) and, in most cases, relatively bright large-scale ambient emission. Each integrated flux measurement was corrected for this background emission by subtracting the product of the median intensity value within a local annulus and the size of the aperture from the direct aperture-flux measurement. The mean and median aperture radii at $70 \mu \mathrm{m}$ are 19 . $^{\prime \prime}$ and $18 .{ }^{\prime \prime} 8$, respectively, as compared to the HiGAL $70 \mu \mathrm{m}$ beam size of 5 !' $8 \times 12$ !" 1 . The mean and median aperture radii at $160 \mu \mathrm{m}$ are $26 . " 3$ and $26 .{ }^{\prime \prime} 7$, respectively, as compared to the HiGAL

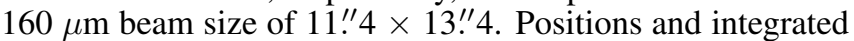
flux values for the Hi-GAL and ATLASGAL data are listed in 
TABLE 5

MIPS $24 \mu \mathrm{M}$ Standard Star FitTed and Catalog FluXes, \& FluX RATIOS

\begin{tabular}{lccccc}
\hline \hline Star $^{a}$ & \multicolumn{2}{c}{ Coordinates $(\mathrm{J} 2000)^{b}$} & Catalog Flux & Fitted Flux & Flux Ratio \\
& RA (h m s) & Dec $\left({ }^{\circ}{ }^{\prime \prime}{ }^{\prime \prime}\right)$ & $(\mathrm{mJy})$ & $(\mathrm{mJy})$ & \\
\hline 1 & $18: 30: 32.40$ & $-09: 35: 47.25$ & $1950(39)$ & $1220(40)$ & 1.60 \\
2 & $18: 30: 12.78$ & $-09: 36: 47.99$ & $1980(36)$ & $1250(38)$ & 1.58 \\
3 & $18: 30: 53.95$ & $-09: 39: 51.27$ & $2960(55)$ & $1870(71)$ & 1.58 \\
4 & $18: 30: 46.32$ & $-09: 32: 28.89$ & $1230(23)$ & $770(23)$ & 1.60 \\
5 & $18: 30: 48.45$ & $-09: 36: 00.11$ & $780(14)$ & $490(17)$ & 1.59 \\
\hline
\end{tabular}

${ }^{a}$ Coordinates and both fitted (this work) and catalog i Gutermuth \& Heyer 2015] flux densities for five bright, isolated point sources in the MIPSGAL Point Source Catalog.

${ }^{\mathrm{b}}$ Listed coordinates are from the MIPS Point Source Catalog Gutermuth \& Heyer 2015.

TABLE 6

MIPS $24 \mu \mathrm{M}$ APERTURE-CORRECTED FitTEd Flux DENSITIES

\begin{tabular}{|c|c|c|c|c|c|}
\hline \multirow[t]{2}{*}{ EGO } & \multirow[t]{2}{*}{ Source } & \multicolumn{2}{|c|}{ Coordinates $(\mathrm{J} 2000)^{a}$} & \multirow{2}{*}{$\begin{array}{l}\text { Fitted Flux } \\
\text { Density (Jy) }\end{array}$} & \multirow{2}{*}{$\begin{array}{l}\text { Aperture-Corrected } \\
\text { Fitted Flux (Jy) }\end{array}$} \\
\hline & & $\mathrm{RA}(\mathrm{h} \mathrm{m} \mathrm{s})$ & $\operatorname{Dec}\left({ }^{\circ} \prime \prime \prime\right)$ & & \\
\hline G10.29-0.13 & a & & & & $<0.28$ \\
\hline \multirow{2}{*}{ G10.34-0.14 } & a & $18: 08: 59.989(0.004)$ & $-20: 03: 34.97(0.06)$ & $0.77(0.03)$ & $1.23(0.08)$ \\
\hline & b & $18: 09: 00.017(0.003)$ & $-20: 03: 28.75(0.05)$ & $1.51(0.04)$ & $2.4(0.1)$ \\
\hline \multirow[t]{2}{*}{ G11.92-0.61 } & $\mathrm{a}$ & $18: 13: 58.065(0.001)$ & $-18: 54: 21.26(0.01)$ & $2.484(0.003)$ & $4.0(0.2)$ \\
\hline & $\mathrm{b}$ & $18: 13: 58.122(0.001)$ & $-18: 54: 14.97(0.01)$ & $2.262(0.003)$ & $3.6(0.2)$ \\
\hline \multirow{3}{*}{ G12.91-0.03 } & a & $18: 13: 48.233(0.002)$ & $-17: 45: 38.19(0.03)$ & $1.11(0.02)$ & $1.77(0.09)$ \\
\hline & b & $18: 13: 48.283(0.001)$ & $-17: 45: 46.21(0.01)$ & $1.50(0.01)$ & $2.4(0.1)$ \\
\hline & $\mathrm{c}$ & $18: 13: 48.469(0.004)$ & $-17: 45: 31.77(0.04)$ & $0.28(0.01)$ & $0.45(0.03)$ \\
\hline \multirow[t]{3}{*}{ G14.33-0.64 } & a & $\ldots$ & & confused & \\
\hline & $\mathrm{b}$ & $\cdots$ & .. & saturated & \\
\hline & $\mathrm{c}$ & $\ldots$ & & confused & \\
\hline G14.63-0.58 & a & $18: 19: 15.221(0.001)$ & $-16: 30: 03.26(0.02)$ & $0.683(0.009)$ & $1.09(0.06)$ \\
\hline \multirow{3}{*}{ G16.59-0.05 } & a & $\ldots$ & $\ldots$ & saturated & $\ldots$ \\
\hline & b & $\ldots$ & $\ldots$ & saturated & $\ldots$ \\
\hline & $\mathrm{c}$ & $\ldots$ & $\ldots$ & confused & \\
\hline \multirow[t]{2}{*}{ G18.89-0.47 } & a & $18: 27: 07.82(0.01)$ & $-12: 41: 35.1(0.2)$ & $0.34(0.04)$ & $0.54(0.07)$ \\
\hline & $\mathrm{b}$ & 18:27:08.45 (0.01) & $-12: 41: 29.5(0.2)$ & $0.64(0.07)$ & $1.0(0.1)$ \\
\hline \multirow[t]{2}{*}{ G19.36-0.03 } & a & $18: 26: 25.782(0.001)$ & $-12: 03: 53.73(0.01)$ & $2.356(0.005)$ & $3.8(0.2)$ \\
\hline & $\mathrm{b}$ & $18: 26: 25.569(0.001)$ & $-12: 03: 48.13(0.01)$ & $3.371(0.006)$ & $5.4(0.3)$ \\
\hline \multirow[t]{2}{*}{ G22.04+0.22 } & a & $18: 30: 34.627(0.001)$ & $-09: 34: 46.24(0.02)$ & $2.23(0.02)$ & $3.6(0.2)$ \\
\hline & b & $18: 30: 33.432(0.001)$ & $-09: 34: 48.39(0.01)$ & $2.79(0.02)$ & $4.4(0.2)$ \\
\hline \multirow[t]{2}{*}{ G28.83-0.25 } & a & $18: 44: 51.136(0.001)$ & $-03: 45: 47.845(0.009)$ & $2.230(0.008)$ & $3.6(0.2)$ \\
\hline & $\mathrm{b}$ & $18: 44: 50.931(0.001)$ & $-03: 45: 56.506(0.009)$ & $2.362(0.008)$ & $3.8(0.2)$ \\
\hline \multirow[t]{2}{*}{ G35.03+0.35 } & a & $\ldots$ & $\ldots$ & saturated & $\ldots$ \\
\hline & $\mathrm{b}$ & $\ldots$ & $\ldots$ & saturated & $\ldots$ \\
\hline
\end{tabular}

${ }^{a}$ Source coordinates are the fitted coordinates returned by imfit. Sources that have $\cdots$ values in place of coordinate values are either undetected at $24 \mu \mathrm{m}$ (G10.29-0.13) or suffer from saturation and/or confusion (G14.33-0.64, G16.59-0.05, G35.03+0.35). Sources with position uncertainties in italics (G11.92-0.61, G19.36-0.03, G22.04+0.22_b) had their coordinates held fixed during the fitting procedure, so the position uncertainties come not from the imfit results but from the uncertainty in the choice of source

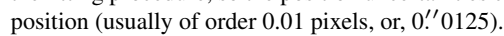

b These are the fitted fluxes directly returned by imfit; they have not been corrected for aperture effects. Sources with " ..." are nondetections at $24 \mu \mathrm{m}$. Sources listed as "saturated" are saturated at $24 \mu \mathrm{m}$. Sources listed as "confused" are not saturated at $24 \mu \mathrm{m}$ but suffer from an angular confusion problem, usually with a nearby saturated source.

c These are the aperture-corrected fitted flux densities, where the applied aperture correction is $1.59 \pm 0.00893$, as calculated in Table 5 . We use the data in this column for constructing our SEDs. Sources listed as " . . " " could not be fit at $24 \mu \mathrm{m}$ due to saturation and/or confusion issues and thus have no fitted flux value to which to apply an aperture correction.

Our far-IR flux uncertainties are the quadrature sum of two values. First, there is the statistical uncertainty of the measurement itself, which we take to be the product of the background noise level $\sigma$ in $\mathrm{Jy} \mathrm{beam}^{-1}$ and the square root of the aperture size in beams. Second, there is the inherent uncertainty of the image due to flux calibration accuracy. Molinari et al. (2016) quote an absolute flux uncertainty of 5\% for the Hi-GAL data, and Schuller et al. (2009) quote an absolute flux uncertainty of $15 \%$ for the ATLASGAL survey. We adopt these values for our uncertainty calculations for the Hi-GAL and ATLASGAL data, respectively.
Far-IR source selection - Beginning at $70 \mu \mathrm{m}$, the fluxes of sources that are not dominant at $37.1 \mu \mathrm{m}$ (sources "b" and "c" for each FOV) begin to decrease, in some cases rapidly. This decrease in flux is usually such that, by either $160 \mu \mathrm{m}$ or $870 \mu \mathrm{m}$, there is only one dominant source at that wavelength. In all cases, that dominant source is spatially coincident with the location of the brightest source at $37.1 \mu \mathrm{m}$. However, the angular resolution of the FIR data worsens as wavelength increases, so even if there are multiple sources present in the FIR images, the angular resolution may be insufficient to separate them. Because of the comparatively low resolution of these images, it is not uncommon to see FIR flux that is spatially coincident with one of the "b" or "c" sources for a given 
EGO, but neither is it clear that the spatial coincidence is not merely a result of resolution limitations. In cases where the morphology of the $70 \mu \mathrm{m}$ or $160 \mu \mathrm{m}$ emission was consistent with a single source, we assigned all the emission in that band to source "a." In cases where it was clear that there were multiple sources present in the Hi-GAL data, we took one of two approaches. First, we attempted to fit the emission using multiple gaussian components using imfit. If we achieved satisfactory fits with this approach, the fitted fluxes of both sources are listed in Table 7. Second, if we could not achieve satisfactory fits with multiple gaussian components, we attempted to estimate the maximum possible amount of flux that could be ascribed to the weaker source. We then performed the photometric procedure described above on the emission as a whole (dominant and weaker source combined) and assigned all of the resulting flux to the dominant $37.1 \mu \mathrm{m}$ source, and added the estimated flux from the weaker source to our uncertainty value for the dominant source. For these cases, the measured fluxes are marked in bold in Table 7 While imperfect, this method does allow us to at least account for the effects of multiple blended sources even when we cannot satisfactorily deblend the emission itself.

Source confusion was not an issue in any of the ATLASGAL images, since the ATLASGAL data a) have an angular resolution that is significantly poorer than any of the other data sets, thus potentially blending any individual sources past the point where one could recognize separate sources, and b) necessarily probe cooler gas. This effectively means that the emission in the ATLASGAL images originates primarily in the outer regions of the parent clump, which is an identifiably larger physical size scale than those probed by the Hi-GAL and our mid- or near-IR data sets. Due to source morphology in the ATLASGAL data and the aforementioned drop in flux in the FIR for sources that are not the dominant source at $37.1 \mu \mathrm{m}$, we attribute all $870 \mu \mathrm{m}$ flux to the single, dominant $37.1 \mu \mathrm{m}$ source in all cases.

The effect of these source-selection criteria is that full SEDs are constructed for the brightest $37.1 \mu \mathrm{m}$ sources (the "a" sources) only, and these SEDS are based on the explicit assumption that these sources are by far the most dominant in the far-IR.

\subsection{Images and Trends}

Figures 1 through 4 show the detected SOFIA 19.7 and $37.1 \mu \mathrm{m}$ emission in the vicinity of each EGO. We detected $37.1 \mu \mathrm{m}$ emission in all twelve fields; in eleven cases, this emission was associated with the EGO. This is in itself a high detection rate. However, we detect an average of only two sources per target, of which only one, on average, is actually associated with the EGO. This suggests that, rather than detecting multiple protostars within each protocluster, we are typically detecting only the dominant source in each EGO. Likewise, we detect $19.7 \mu \mathrm{m}$ emission in nine of our twelve fields, but it is only associated with the target EGO in eight cases. We detect more $19.7 \mu \mathrm{m}$ emission toward sources that are not associated with the target EGOs than emission toward sources that are (10/18 not associated versus 8/18 that are). At $19.7 \mu \mathrm{m}$, we still detect an average of two sources per target. Taken together, these trends suggest that our target protoclusters are still quite young and/or deeply-embedded; this would explain the trend of overall dominance by a single source, as well as the poorer detection rate of even these dominant sources at $19.7 \mu \mathrm{m}$.

Of our $37.1 \mu \mathrm{m}$ sources, all but one are located entirely within the $25 \%$ ATLASGAL contours of the clump associated with the target EGO, for a total of $2337.1 \mu \mathrm{m}$ sources within eleven ATLASGAL clumps (G10.29-0.13 has no $37.1 \mu \mathrm{m}$ emission toward the EGO itself, so its ATLASGAL clump is not counted). This is an average of slightly more than two mid-infrared sources per clump. The one $37.1 \mu \mathrm{m}$ source not located within an ATLASGAL clump is G14.33-0.64_b, which has some extended emission within the $870 \mu \mathrm{m}$ contours but is centered outside of it; our source G14.33-0.64_b is the known H II region IRAS 18159-1648 (Jaffe et al. 1982).

Eleven of our sources are located in IRDCs; the only exception is G16.59-0.05. Eleven sources are known to be coincident with $6.7 \mathrm{GHz} \mathrm{CH}_{3} \mathrm{OH}$ masers (references for maser detections are in the tablenotes of Table 11; the remaining source, G14.33-0.64, has no published 6.7 GHz data at the time of writing. Three sources - G10.29-0.13 and G10.34-0.14 (near the W31 H II region G10.32-00.15, see Westerhout 1958), and G28.83-0.25 (near N49, see Wink et al. 1982) - are adjacent to are known H II or UCH II regions.

\subsection{Mid-infrared Multiplicity}

There is some evidence of multiplicity at mid-infrared wavelengths for nearly all of our targets, with G10.29-0.13 (lacking any mid-IR detection) and G14.63-0.58 being the only exceptions. The evidence for mid-IR multiplicity for the other sources falls generally into two categories: individual EGO-related sources (i.e. within the boundaries of extended $4.5 \mu \mathrm{m}$ emission) that have unresolved substructure at the angular resolution of our SOFIA data, and sources that have nearby $\left(\lesssim 10^{\prime \prime}\right) 37.1 \mu \mathrm{m}$ detections which are not within the extended 4.5um emission of the EGO, and whose association with the EGO is unclear. We discuss each category in greater detail in the following sections. The naming convention of the new detections is described in $\$ 3.1$.

\subsubsection{EGO Sources with Unresolved Substructure at $37.1 \mu \mathrm{m}$}

The dominant EGO-related sources in G11.92-0.61, G14.33-0.58, G28.83-0.25, G35.03+0.35 exhibit elongated, unresolved $37.1 \mu \mathrm{m}$ emission suggestive of multiplicity at scales $\lesssim 5^{\prime \prime}$ (the SOFIA angular resolution is 3.'4). Below we explore how the mid-IR emission compares to existing high resolution centimeter to millimeter data. This comparison helps inform the nature of the emission at each wavelength. Mid-IR emission may trace both hot cores and outflow cavities, while centimeter emission can trace both free-free emission (e.g. H II region, ionized jet) and the long-wavelength end of the Rayleigh-Jeans tail of dust emission. Millimeter observations (in this context) primarily serve to identify individual cores from dust continuum emission. By comparing the emission from these different wavelength regimes, we can attempt to disentangle the possible sources of mid-IR emission in these objects.

G11.92-0.61 - G11.92-0.61 is elongated roughly N-S at $37.1 \mu \mathrm{m}$, and shows two distinct sources at $19.7 \mu \mathrm{m}$ which lie along the axis of the $37.1 \mu \mathrm{m}$ elongation (Fig. 1). The southern and northern mid-IR sources (G11.92-0.61_a and G11.92-0.61_b) are coincident with the (sub)millimeter protostellar sources MM1 and MM3, respectively (Cyganowski et al. 2011a, 2017). Both MM1 and MM3 are associated with $6.7 \mathrm{GHz} \mathrm{CH} \mathrm{CH}_{3} \mathrm{OH}$ masers (a signpost of massive star formation Cyganowski et al. 2009, 2011a), and both have also been detected at centimeter wavelengths (the centimeter sources are designated CM1 and CM2 Cyganowski et al. 
TABLE 7

HI-GAL $70 \mu \mathrm{M} \& 160 \mu \mathrm{M}$ AND ATLASGAL $870 \mu \mathrm{M}$ FLUX DENSITIES

\begin{tabular}{|c|c|c|c|c|c|}
\hline EGO & Source & $\begin{array}{c}\mathrm{Hi}^{-\mathrm{GAL}^{a}} \\
70 \mu \mathrm{m} \text { Notes }\end{array}$ & $\underset{(\mathrm{Jy})}{\mu \mathrm{Flux}^{b}}$ & $\underset{(\mathrm{Jy})}{160 \mathrm{~m}^{2} \text { Flux }^{b}}$ & $\underset{(\mathrm{Jy})}{870 \mu \mathrm{m} \text { Flux }}$ \\
\hline G10.29-0.13 & $\mathrm{a}$ & $\ldots$ & $75(6)$ & $298(30)$ & $5.0(0.8)$ \\
\hline G10.34-0.14 & $\begin{array}{l}\mathrm{a} \\
\mathrm{b}\end{array}$ & $\begin{array}{l}\cdots \\
\text { assuming all emission from a }\end{array}$ & $280(20)$ & $590(49)$ & $\begin{array}{c}6(1) \\
\cdots\end{array}$ \\
\hline G11.92-0.61 & $\begin{array}{l}\mathrm{a} \\
\mathrm{b}\end{array}$ & $\begin{array}{l}\cdots \\
\text { assuming all emission from a }\end{array}$ & $\begin{array}{c}640(33) \\
\cdots\end{array}$ & $\begin{array}{c}980(58) \\
\cdots\end{array}$ & $\begin{array}{c}11(2) \\
\cdots\end{array}$ \\
\hline G12.91-0.03 & $\begin{array}{l}\mathrm{a} \\
\mathrm{b} \\
\mathrm{c}\end{array}$ & $\begin{array}{l}\ldots \\
\text { assuming all emission from a } \\
\text { assuming all emission from a }\end{array}$ & $\begin{array}{l}96(6) \\
\cdots \\
\cdots\end{array}$ & $\begin{array}{c}270(52) \\
\cdots \\
\cdots\end{array}$ & $\begin{array}{l}7(1) \\
\cdots \\
\cdots\end{array}$ \\
\hline G14.33-0.64 & $\begin{array}{l}\mathrm{a} \\
\mathrm{b} \\
\mathrm{c}\end{array}$ & $\begin{array}{l}\ldots \\
\text { assuming all emission from a } \\
\text { assuming all emission from a }\end{array}$ & $\begin{array}{c}1130(190) \\
\ldots \\
\cdots\end{array}$ & $\begin{array}{c}1940(120) \\
\cdots \\
\cdots\end{array}$ & $\begin{array}{c}32(4) \\
\cdots \\
\cdots\end{array}$ \\
\hline G14.63-0.58 & $\mathrm{a}$ & $\cdots$ & $130(8)$ & $390(30)$ & $15(2)$ \\
\hline G16.59-0.05 & $\begin{array}{l}\mathrm{a} \\
\mathrm{b} \\
\mathrm{c}\end{array}$ & $\begin{array}{l}\ldots \\
\text { assuming all emission from a } \\
\text { assuming all emission from a }\end{array}$ & $\begin{array}{c}490(26) \\
\ldots \\
\ldots\end{array}$ & $\begin{array}{c}740(48) \\
\cdots \\
\cdots\end{array}$ & $\begin{array}{c}9(1) \\
\cdots \\
\cdots\end{array}$ \\
\hline G18.89-0.47 & a & $\begin{array}{l}\text { sources } a \text { and } b \text { fit with } \\
\text { imfit } \\
\text { sources a and } b \text { fit with } \\
\text { imfit }\end{array}$ & $\begin{array}{c}48(2) \\
19.6(0.2)\end{array}$ & 180 (27) & $\begin{array}{c}10(2) \\
\ldots\end{array}$ \\
\hline G19.36-0.03 & $\begin{array}{l}\mathrm{a} \\
\mathrm{b}\end{array}$ & $\begin{array}{l}\ldots \\
\text { assuming all emission from a }\end{array}$ & $250(14)$ & $470(41)$ & $\begin{array}{c}8(1) \\
\cdots\end{array}$ \\
\hline $\mathrm{G} 22.04+0.22$ & $\mathrm{~b}$ & $\begin{array}{l}\text { sources } a \text { and } b \text { fit with } \\
\text { imfit } \\
\text { sources a and } b \text { fit with } \\
\text { imfit }\end{array}$ & $\begin{array}{c}204(10) \\
59(3)\end{array}$ & $400(94)$ & $5.9(0.8)$ \\
\hline G28.83-0.25 & $\begin{array}{l}\mathrm{a} \\
\mathrm{b}\end{array}$ & $\begin{array}{l}\ldots \\
\text { assuming all emission from a }\end{array}$ & $\begin{array}{c}510(65) \\
\ldots\end{array}$ & $\begin{array}{c}870(60) \\
\ldots\end{array}$ & $\begin{array}{c}10(1) \\
\cdots\end{array}$ \\
\hline G35.03+0.35 & $\begin{array}{l}\mathrm{a} \\
\mathrm{b}\end{array}$ & $\begin{array}{l}\cdots \\
\text { assuming all emission from a }\end{array}$ & $\begin{array}{c}1350(71) \\
\ldots\end{array}$ & $\begin{array}{c}1230(84) \\
\ldots\end{array}$ & $\begin{array}{c}8(1) \\
\cdots\end{array}$ \\
\hline
\end{tabular}

a This column addresses the confusion of our sources at the $70 \mu \mathrm{m}$ wavelength and angular resolution. Sources with the note "assuming all emission from a" do have $70 \mu \mathrm{m}$ emission coincident with the position of source b and/or c, but we assume the emission to be entirely from or significantly dominated by source a. Sources with the note "sources a and b fit with imf it" have emission coincident with both source a and source b, and there were two emission regions sufficiently distinguishable at $70 \mu \mathrm{m}$ to be fit with the imfit tool. These notes only apply to the $70 \mu \mathrm{m}$ data.

$\mathrm{b}$ The sources in bold in these two columns suffer from confusion at either 70 or $160 \mu \mathrm{m}$, and were not sufficiently wellseparated to be successfully fit with two components imfit. In these cases, the uncertainties of the flux densities are increased to reflect this effect. The precise method by which the uncertainties account for the confusion issue is discussed in detail in-text in $\$ 3.2 .3$

2011b, Cyganowski et al. 2014; Moscadelli et al. 2016; Ilee et al. 2016; Towner et al. 2017).

To further explore how sensitive the SOFIA data are to the presence of multiple protostellar sources, we turn to highangular resolution, high-sensitivity millimeter data. Atacama Large Millimeter/submillimeter Array (ALMA) observations of G11.92-0.61 (1.05 mm, 0." $49 \times 0$ ". 34 synthesized beam) by Cyganowski et al. (2017) reveal at least eight $1.05 \mathrm{~mm}$ sources within a $5^{\prime \prime}$ radius of the peak of the $37.1 \mu \mathrm{m}$ emission, two of which correspond to MM1 and MM3). Of these eight, the authors estimate that six are low-mass objects, one is intermediate- or high-mass (MM3), and one is high-mass (MM1). Indeed, follow-up observations of MM1 at $1.3 \mathrm{~mm}$

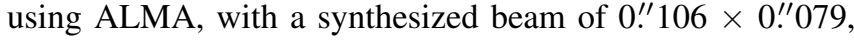
find that this source is likely a proto-O star whose circumstellar disk dynamics yield an enclosed mass of $\mathrm{M}_{\mathrm{enc}} \sim 40 \pm$ $5 M_{\odot}$ (Ilee et al. 2018). These radio data suggest that the mid-
IR morphology of G11.92-0.61 is dominated by the two intermediate to massive protostellar sources (MM1 and MM3), rather than, e.g., a poorly-resolved outflow cavity. This result also indicates that our SOFIA data are sensitive to massive protostellar multiplicity, though as expected the mid-IR data are not sensitive to lower mass (and luminosity) protocluster members (also see $\$ 4.3 .1$.

G14.33-0.64 - The dominant EGO-related source G14.33-0.64_a (Fig. 2) is slightly elongated N-S at $37.1 \mu \mathrm{m}$, and there is a $19.7 \mu \mathrm{m}$ detection associated with the northern portion of the elongation. The brightest component, G14.33-0.64_b, is coincident with the known evolved H II region IRAS 18159-1648. In order to achieve satisfactory fits to the $37.1 \mu \mathrm{m}$ emission toward G14.33-0.64_a, it is necessary to fit a third component. G14.33-0.64_c is located $\sim 4^{\prime \prime}$ east-southeast of G14.33-0.64_a, is faint at both $37.1 \mu \mathrm{m}$ and $24 \mu \mathrm{m}$, and is undetected at $19.7 \mu \mathrm{m}$. 
Towner et al. (2017) report significant JVLA $1.3 \mathrm{~cm}\left(4 .^{\prime \prime} 6 \times\right.$ 2."5 beam) emission coincident with G14.33-0.64_a and the $\mathrm{H}$ II region G14.33-0.64_b, as well as a marginal detection at the location of G14.33-0.64_c (to within stated position uncertainties), though they were unable to get a satisfactory fit for its (weak) $1.3 \mathrm{~cm}$ flux density. Unfortunately, there are no published high-angular resolution millimeter continuum data for this source, though the mid-IR and centimeter data hint that there may be at least two massive protostars coincident with the EGO.

G28.83-0.25 - G28.83-0.25_a is elongated E-W, consistent with unresolved substructure; this source is not detected at $19.7 \mu \mathrm{m}$ (Fig. 4). Based on the $37.1 \mu \mathrm{m}$ emission alone, it is unclear whether this elongation is indicative of multiple unresolved sources or is due to a different cause, such as an unresolved outflow cavity. Interestingly, the elongation follows the same axis as the extended $4.5 \mu \mathrm{m}$ emission, which is thought to be due to outflow activity. Towner et al. (2017) detect two $1.3 \mathrm{~cm}$ continuum sources toward this EGO: one is coincident with the peak of the $37.1 \mu \mathrm{m}$ emission (called CM2), and one that is coincident with the extended "spur" on the western edge of G28.83-0.25_a (called CM1). Both $1.3 \mathrm{~cm}$ sources are unresolved at the angular resolution of the $1.3 \mathrm{~cm}$ data $\left(\sim 3^{\prime \prime}\right)$. Both sources are also reported by Cyganowski et al. (2011b) at $3.6 \mathrm{~cm}$ with $\sim 1^{\prime \prime}$ resolution. Comparing the two centimeter wavelengths, Towner et al. (2017) suggest that either CM2 has a steeper free-free SED than CM1 or has a higher contribution from dust. If free-free emission is present, then the E-W elongation at both $1.3 \mathrm{~cm}$ and $37.1 \mu \mathrm{m}$ suggests that this emission could be due to an ionized jet. In the absence of higher-resolution MIR images, and comparable millimeter wavelength data we cannot definitively attribute the elongation in this source to either outflow activity or multiple unresolved protostellar sources.

G35.03+0.35 - The $37.1 \mu \mathrm{m}$ emission for G35.03+0.35 is elongated NE-SW and is indicative of at least two unresolved sources (Fig. 4); at $19.7 \mu \mathrm{m}$, the emission is resolved into two distinct sources, which lie along the major axis of the $37.1 \mu \mathrm{m}$ elongation. When observed at $1.3 \mathrm{~cm}$ with similar angular resolution $\left(\sim 3^{\prime \prime}\right)$ to the $37.1 \mu \mathrm{m}$ data, the brighter $37.1 \mu \mathrm{m}$ source, G35.03+0.35_a, is coincident with compact, unresolved $1.3 \mathrm{~cm}$ continuum emission as reported by Brogan et al. (2011); Towner et al. (2017). However, higher angular resolution $3.6 \mathrm{~cm}$ VLA observations $(\sim 1)$ resolve the continuum emission for G35.03+0.35 into at least five distinct, compact centimeter sources (Cyganowski et al. 2011b). Four of these $3.6 \mathrm{~cm}$ sources are coincident with the brighter $37.1 \mu \mathrm{m}$ source, G35.03+0.35_a, and the unresolved $1.3 \mathrm{~cm}$ source. The two strongest of these $3.6 \mathrm{~cm}$ sources (CM1 and CM2), trace a known ultra-compact H II region (Kurtz et al. 1994), and likely a hyper-compact H II region (Cyganowski et al. 2011b), respectively. Therefore, G35.03+0.35_a, harbors at least two massive protostars. With ALMA at $0.87 \mathrm{~mm}$, Beltrán et al. (2014) also detect CM1 and CM2, but not CM3, suggesting the latter is not a protostar. More recent high angular resolution JVLA observations of G35.03+0.35 (0.' 34 resolution) and analysis of the SEDs by Sanna et al. (2019, submitted), suggest that the hyper-compact H II region CM2 is driving a powerful outflow and that $\mathrm{CM} 3$ corresponds to jet emission launched from CM2. The fifth $3.6 \mathrm{~cm}$ source (denoted CM3) lies in the direction of the weaker 19.7 and $37.1 \mu \mathrm{m}$ detections, G35.03+0.35_b, but the $19.7 \mu \mathrm{m}$ source appears to extend further to the NE than the $3.6 \mathrm{~cm}$ emission. Thus, G35.03+0.35_b may be tracing an outflow cavity that extends to the NE of G35.03+0.35_a (and the likely powering source CM2).

\subsection{2. $37.1 \mu m$ Detections For Which the Association with EGOs is Unclear}

Many of the non-dominant $37.1 \mu \mathrm{m}$ sources in our sample (the "b" and "c" sources) lie close to $\left(\lesssim 10^{\prime \prime}\right)$ the dominant $37.1 \mu \mathrm{m}$ source but outside the bounds of the $4.5 \mu \mathrm{m}$ extended emission. They also typically are redder in color than the "a" sources. The association of these non-dominant MIR sources with the EGOs is unclear, but understanding this association is an important component of understanding the mid-IR multiplicities in this sample.

One example of such a case is EGO G19.36-0.03, which has two sources identified in Table 3 They are fully separable at $19.7 \mu \mathrm{m}$ but only marginally separable at $37.1 \mu \mathrm{m}$ (Fig. 3). The fainter source at $37.1 \mu \mathrm{m}$, G19.36-0.03_b, lies outside the extended $4.5 \mu \mathrm{m}$ emission of the EGO. Both sources have unresolved $1.3 \mathrm{~cm}$ continuum counterparts, reported by Towner et al. (2017). G19.36-0.03_b is the stronger source at $1.3 \mathrm{~cm}$, and also has compact emission at $3.6 \mathrm{~cm}$ (Cyganowski et al. 2011b) with $\sim 1^{\prime \prime}$ resolution. It is also coincident with MIPS $24 \mu \mathrm{m}$ and IRAC emission, and is associated with a line of $44 \mathrm{GHz}$ Class I $\mathrm{CH}_{3} \mathrm{OH}$ masers; Cyganowski et al. (2011b) suggest that it is therefore a candidate for an expanding $\mathrm{H}$ II region. If G19.36-0.03_b is indeed associated with the EGO, then the multiplicity of massive protostars in this EGO is 2. Furthermore, this would make G19.36-0.03 an example of a massive protocluster in which multiple stages of high-mass star formation are occurring simultaneously, as noted by Cyganowski et al. (2011b). In this case, the classification of the EGO protocluster is significantly impacted by the association (or lack thereof) between the two $37.1 \mu \mathrm{m}$ detections.

Other such cases in our sample include G10.34-0.14_b, G18.89-0.47_b, and G12.91-0.03_c. If every one of our "b" and "c" sources is truly associated with an EGO (except the $\mathrm{H}$ II region IRAS 18159-1648), then our average multiplicity of massive sources in this sample - at $37.1 \mu \mathrm{m}$ with $\sim 3^{\prime \prime}$ resolution - is 1.9 . If only half are truly associated, the average multiplicity is 1.4 . These values are roughly in line with the results of Rosero et al. (2018), who find no strong evidence of high mulitplicity ( $>2$ massive sources) in a subset of similar massive protostellar sources from the SOFIA Massive Star Formation Survey (SOMA) sample (see De Buizer et al. 2017. and Section 4.3.4 of this work, for a discussion of the SOMA sample and subsamples).

In order to properly address this multiplicity question, additional observations are needed along the lines of those described above for G11.92-0.61 and G35.03+0.35. Such observations must be able to distinguish individual dust cores ( $\lesssim 0.02$ pc spatial resolution) and establish the nature (ionized jet, H II region, synchrotron, etc.) of the centimeterwavelength emission. The former allow the identification of individual sources, and the latter allow the differentiation between ionized jets and $\mathrm{HC} \mathrm{HII}$ regions. Indeed, we have observations underway for the majority of the EGOs in this sample with sub-arcsecond resolution in the JVLA C- and Kbands, and with ALMA Band 3 and Band 6. The results of these observations will be published in future work.

\section{ANALYSIS}


In order to estimate temperature and mass of the parent clumps, we performed greybody fits to the SEDs in order to derive representative temperatures for each EGO. Our greybody fits use only the far-IR (Hi-GAL and ATLASGAL) data for each source, and are used to derive dust temperatures that were then used to calculate clump masses based on the ATLASGAL $870 \mu \mathrm{m}$ integrated flux densities. In order to independently assess gas temperature in these clumps, we also examine the gas kinetic temperatures determined by Cyganowski et al. (2013), as described below. Table 8 shows the $\mathrm{NH}_{3}$ and greybody temperature results for each source, along with corresponding estimated masses. The last column also lists the FIR luminosity of each EGO, as returned by the greybody fits.

In order to determine $L_{\star}$ for each target, we fit the SEDs with several different publicly-available SED models including those published in Robitaille et al. (2006), Robitaille (2017), and Zhang \& Tan (2018). The different underlying assumptions and components for each model are described below, in order of model publication date. Figure 5 and Figures 7 through 18 show the SEDs and model fits in the following order for each source: six panels showing Robitaille (2017) models, one panel showing Robitaille et al. (2006) models, and one panel showing Zhang \& Tan (2018) models. Figures 7 through 18 are located in Appendix A.

\subsection{Temperature and Mass From Dust and $\mathrm{NH}_{3}$ Emission}

In order to determine the mass of the ATLASGAL clumps (the mass reservoirs) in which our sources are located, we need to know the temperature of the emitting material. This is typically accomplished either by fitting models to molecular line emission (e.g. $\mathrm{NH}_{3}, \mathrm{CH}_{3} \mathrm{CN}$ ) or by fitting greybody functions to far-IR dust emission. For this work, we chose to employ each method separately and compare results.

For the gas temperature, we adopt the single-component $\mathrm{NH}_{3}$ fit results of Cyganowski et al. (2013), who performed a $\mathrm{H}_{2} \mathrm{O}$ maser and $\mathrm{NH}_{3}(1,1)$ through $(3,3)$ inversion-line survey of 94 GLIMPSE-identified EGOs using the Nobeyama Radio Observatory 45-meter telescope. The kinetic temperature $\left(T_{k i n}\right)$ results from Cyganowski et al. (2013) are shown in Table 8 The Cyganowski et al. (2013) $\mathrm{NH}_{3}$ temperatures for our sample ${ }^{12}$ have minimum, maximum, and median values of $20.5,29.5$, and $25.8 \pm 2.5 \mathrm{~K}$, respectively, where the uncertainty on the median is the MAD.

In order to estimate dust temperature and derive clump mass, we used the Python package lmfit to fit a series of greybody curves to our far-IR (70 $\mu \mathrm{m}, 160 \mu \mathrm{m}$, and $870 \mu \mathrm{m}$ ) flux densities and thereby derive a temperature $\mathrm{T}_{\text {dust }}$ for each source. During this procedure, the grain opacity spectral in$\operatorname{dex}(\beta)$ was fixed at 1.7 (Brogan et al. 2016; Sadavoy et al. 2016). We defined a grid of $\mathrm{T}_{\text {dust }}$ ranging from $18.0 \mathrm{~K}$ to 40.0 $\mathrm{K}$ in steps of $1.0 \mathrm{~K}$. For each value of $\mathrm{T}_{\text {dust }}$, we fit for the opacity at a reference wavelength and computed the corresponding luminosity $\left(\mathrm{L}_{F I R}\right)$. The best-fit temperature was defined as the temperature for which $\chi^{2}$ was closest to 1 , and the best-fit luminosity was the luminosity corresponding to this best-fit temperature. Then, we calculated the total gas mass of each source as

12 While Cyganowski et al. (2013) note that the $\mathrm{NH}_{3}(3,3)$ masers detected by Brogan et al. (2011) in G35.03+0.35 (Brogan et al. 2011) are not readily distinguishable as a non-thermal contribution in the Nobeyama data, contamination by $(3,3)$ masers is unlikely to significantly impact the fitted temperatures.

$$
M_{\text {gas }}=R\left(\frac{F_{\nu} D^{2}}{B_{\nu}\left(T_{\text {dust }}\right) \kappa_{\nu}}\right)\left(\frac{\tau}{1-e^{-\tau}}\right)
$$

where $R=100$ is the gas-to-dust mass ratio, $F_{\nu}$ is the measured $870 \mu \mathrm{m}$ flux density of the source, $D$ is the distance to the source, $B_{\nu}\left(T_{\text {dust }}\right)$ is the blackbody function, $\kappa_{\nu}$ is the dust opacity, and $\tau$ is the optical depth at $870 \mu \mathrm{m}$. The dust opacity was fixed at $\kappa_{870 \mu m}=1.85 \mathrm{~cm}^{2} \mathrm{~g}^{-1}$, which is the value Schuller et al. (2009) interpolate from Table 1 of Ossenkopf \& Henning (1994) and which is employed by Cyganowski et al. (2017) for their calculation of the mass reservoir of G11.92-0.61. In all cases, the fitted opacity is sufficiently small at $870 \mu \mathrm{m}$ that $\frac{\tau}{1-e^{-\tau}} \approx 1$. We calculate mean and median $T_{\text {dust }}=25.8 \mathrm{~K}$ and $26.0 \mathrm{~K}$, respectively, with a standard deviation of $2.9 \mathrm{~K}$ and a MAD of $1.5 \mathrm{~K}$.

While the mean and median values of the two temperature estimates are in statistical agreement, there is a general trend that the temperatures calculated using $\mathrm{NH}_{3}$ inversion transitions are slightly lower than those calculated from FIR dust emission. The median difference between the dust- and gasderived temperatures is only $0.92 \mathrm{~K}$, so the trend is weak and further, more precise investigation is needed in order to make a definitive statement about the implications of such a trend. However, it should be noted that this is in broad agreement with the trends noted by König et al. (2017) and Giannetti et al. (2017) for the ATLASGAL Top100 sample ${ }^{13}$, Both authors find that dust and $\mathrm{NH}_{3}$ temperatures are well-correlated for massive star-forming clumps overall, but that $\mathrm{NH}_{3}$ emission tends to trace gas that is warmer than dust in very cold clumps $(\lesssim 15 \mathrm{~K})$, and gas that is cooler than dust in warmer clumps $(>15 \mathrm{~K})$.

The median EGO greybody-derived $T_{\text {dust }}(26.0 \mathrm{~K})$ is similar to that of the the median dust temperature of the Top 100 sample (24.7 K, see Table 2 in König et al. 2017). However, König et al. (2017) sort the Top100 sources into four subcategories, of which the "IR-weak" $\left(F_{24 \mu m}<2.6 \mathrm{Jy}\right.$, median $T_{\text {dust }}$ $=21.4 \mathrm{~K})$ and "IR-bright" $\left(F_{24 \mu m}>2.6 \mathrm{Jy}\right.$, median $T_{\text {dust }}=28.2$ K) samples are the most similar to the EGO sample. Indeed, when scaled appropriately for distance ${ }^{14}$, half of our sources have $F_{24} \mu m<2.6 \mathrm{Jy}$, and half have $F_{24 \mu m}>2.6 \mathrm{Jy}$. Interestingly, we find that the EGO median dust temperature also falls in between the median $T_{\text {dust }}$ of the IR-weak and IR-bright populations, though it is closer to the IR-bright $T_{\text {dust }}$. However, it is notable that the temperature ranges of the two categories are broad: 11.7 to $26.2 \mathrm{~K}$ for IR-weak and 21.9 to $35.4 \mathrm{~K}$ for IR-bright, with overlap in the 21.9 to $26.2 \mathrm{~K}$ range. Indeed, approximately $50 \%$ of the IR-weak and $40 \%$ of the IR-bright sources fall in this overlapping range of $T_{\text {dust }}$, so these two subcategories are not distinct with regard to the dust temperature. Interestingly, all of the EGO $T_{\text {dust }}$ fall within the Top100 IR-bright range, with the majority (75\%) also falling in the overlap region. Two of the EGO-12 sources, G14.63-0.58 and G18.89-0.47, are included in the Top100 sample, and

13 König et al. 2017) and Giannetti et al. (2017) are the third and fifth papers, respectively, in a series on the ATLASGAL Top100 sample, which consists of 110 of the brightest submillimeter sources in the ATLASGAL compact source catalog selected to span a full range of evolutionary stages. For a description of the sample properties and selection criteria, see Giannetti et al. (2014).

${ }_{14}$ König et al. (2017) and Giannetti et al. 2017) both adopt 2.6 Jy as the $24 \mu \mathrm{m}$ IR-weak/bright cutoff as that is the flux density of a B3 star at $4 \mathrm{kpc}$; for the flux comparison above, we scale the $24 \mu \mathrm{m}$ fluxes listed in Table 6 for a distance of $4 \mathrm{kpc}$. 
TABLE 8

Temperature \& Mass From Greybody Fits and Single-dish $\mathrm{NH}_{3} \mathrm{OBSERVATIONS}$

\begin{tabular}{|c|c|c|c|c|c|c|}
\hline \multirow[t]{2}{*}{ EGO } & \multirow{2}{*}{$\begin{array}{c}\text { Distance }^{a} \\
\quad(\mathrm{kpc})\end{array}$} & \multicolumn{2}{|c|}{ Temperatures (K) } & \multicolumn{2}{|c|}{ Masses $\left(\mathrm{M}_{\odot}\right)$} & \multirow{2}{*}{$\begin{array}{c}\mathrm{L}_{F I R}^{b} \\
\left(10^{3} L_{\odot}\right)\end{array}$} \\
\hline & & $\mathrm{T}_{d u s t}^{c}$ & $\mathrm{~T}_{N H_{3}}$ & Greybody & $\mathrm{NH}_{3}$-derived ${ }^{d}$ & \\
\hline G10.29-0.13 & 1.9 & $24(1)$ & $21.19(0.17)$ & 76 & 91 & 0.90 \\
\hline G10.34-0.14 & 1.6 & $26(1)$ & $28.23(0.38)$ & 62 & 56 & 1.42 \\
\hline G11.92-0.61 & $3.38_{-0.27}^{+0.33}(3.5)$ & $27(1)$ & $26.27(0.19)$ & 450 & 466 & 12.76 \\
\hline G12.91-0.03 & -4.5 & $23(1)$ & $23.56(0.31)$ & 649 & 627 & 5.49 \\
\hline G14.33-0.64 & $1.13_{-0.11}^{+0.14}(2.3)$ & $29(2)$ & $25.26(0.17)$ & 132 & 159 & 2.84 \\
\hline G14.63-0.58 & $1.83_{-0.07}^{+0.08}(1.9)$ & $22(1)$ & $20.76(0.32)$ & 234 & 254 & 1.31 \\
\hline G16.59-0.05 & $3.58_{-0.27}^{+0.32}(4.2)$ & $26(1)$ & $20.51(0.38)$ & 456 & 636 & 10.57 \\
\hline G18.89-0.47 & 4.2 & $22(1)$ & $28.24(0.19)$ & 879 & 625 & 3.04 \\
\hline G19.36-0.03 & 2.2 & $26(1)$ & $24.90(0.31)$ & 147 & 155 & 2.61 \\
\hline $\mathrm{G} 22.04+0.22$ & 3.4 & $26(1)$ & $26.71(0.49)$ & 257 & 248 & 4.90 \\
\hline G28.83-0.25 & 4.8 & $26(1)$ & $28.27(0.50)$ & 851 & 761 & 20.62 \\
\hline G35.03+0.35 & $2.32_{-0.20}^{+0.24}(3.2)$ & $33(1)$ & $29.54(0.92)$ & 119 & 138 & 9.98 \\
\hline
\end{tabular}

${ }^{a}$ Distances shown without uncertainties are estimated from the LSRK velocity and the Galactic rotation curve parameters from Reid et al. (2014). Parallax distances (with their uncertainties) are given where available from Reid et al. 2014), and references therein, with the kinematic distance in parentheses for comparison. All kinematic distances are the near distance.

${ }^{\mathrm{b}}$ Returned by the greybody fits to Hi-GAL $70 \mu \mathrm{m} \& 160 \mu \mathrm{m}$ and ATLASGAL $870 \mu \mathrm{m}$ integrated flux densities.

c The $T_{\text {dust }}$ was derived from greybody fits using a grid of parameters, in which temperature goes in steps of 1 $\mathrm{K}$. Therefore, the uncertainties for all greybody-derived temperatures are $1 \mathrm{~K}$, except for G14.33-0.64, which had three adjacent temperatures with the same $\chi^{2}$ value. Here we present the median of those three temperatures, and increase the uncertainty for this source to $2 \mathrm{~K}$

${ }^{\mathrm{d}}$ These are the masses calculated from the ATLASGAL $870 \mu \mathrm{m}$ fluxes assuming $T_{d u s t}=T_{\text {kin }}\left(\mathrm{NH}_{3}\right)$.

both are classified as IR-weak. This is consistent with our distance-scaled $F_{24 \mu m}$ discussed above.

The strong overlap of the EGO and IR-bright $T_{\text {dust }}$ suggests that the two samples may be drawn from the same parent population, but the clustering of the EGO $T_{\text {dust }}$ in the IR-weak/IRbright overlap region is nontrivial and cannot be discounted. Based on these competing factors, it seems likely that either a) EGOs preferentially lie somewhere between the IR-weak and IR-bright samples in temperature space, or b) EGOs represent the colder end of the IR-bright sample, but are still only a subset of the IR-bright population and do not constitute a separate population. Unfortunately, König et al. (2017) do not correlate the Top100 sources with sources in the EGO catalogs of Cyganowski et al. (2008) and Chen et al. (2013), so we cannot say definitively whether or not EGOs are well-represented in the current Top100 sample. If they are, this could explain the apparent overlap in population, and if not, a comparison of the properties of EGOs with the Top100 sample would be warranted.

The Cyganowski et al. (2013) $\mathrm{NH}_{3}$ temperatures for our sample are slightly warmer than the $\mathrm{NH}_{3}$ temperatures for both the "IR-weak" and "IR-bright" subcategories of the Top100 sample ( $\sim 18 \mathrm{~K}$ and $\sim 22 \mathrm{~K}$, respectively; Giannetti et al. 2017). The masses calculated from the two temperatures have mean and median differences of $1.8 \%$ and $4.3 \%$, respectively, with a maximum difference of $40.6 \%$ and a standard deviation of $17.2 \%$. The mean and median of the ratio of greybody-derived to ammonia-derived mass are $98.2 \%$ and $95.7 \%$, respectively, again with a standard deviation of $17.2 \%$. This difference might indicate that the greybody temperatures systematically produce slightly lower masses than the $\mathrm{NH}_{3}$ derived masses, in agreement with the trend noted above that $\mathrm{NH}_{3}$ generally traces cooler material than dust except in the very coldest environments. However, we do not find that those sources where greybody fits produce lower masses than the $\mathrm{NH}_{3}$ fits are systematically the warmest or coldest clumps (using either the greybody or $\mathrm{NH}_{3}$ temperatures). Furthermore, the high standard deviations on both of these numbers sug- gest that the two sets of masses are effectively identical; there is no statistically-significant trend biasing one mass estimate higher than the other. Given that a sample size of 12 is still well within the regime of small-number statistics, we would strongly caution against over-extrapolating from these particular results - either for or against a particular mass-ratio trend.

Overall, the luminosities we calculate from the greybody fits to our sources are in good agreement with results published by other teams for these or similar sources. Moscadelli et al. (2016) constructed SEDs for 40 high-mass YSOs, including four of our targets (G11.92-0.61, G14.63-0.58, G16.59-0.05, and G35.03+0.35), using integrated fluxes from the online image archives for the MSX (Egan et al. 2003) and WISE (Wright et al. 2010) surveys and the point-source catalogs of IRAS (Neugebauer et al. 1984) and SCUBA (Di Francesco et al. 2008). They calculated bolometric luminosity for each source by directly integrating the area under the SED curve. The $L$ from our greybody fits for these four EGOs agree with the Moscadelli et al. (2016) luminosities within $\pm 20 \%$, with no trend toward over- or under-estimation. Urquhart et al. (2018) conducted a systematic analysis of the properties of $\sim 8,000$ dense clumps in the ATLASGAL Compact Source Catalog (CSC), including deriving $L, M$, and temperature. They performed automated aperture photometry for each clump using the ATLASGAL $870 \mu \mathrm{m}$ maps in conjunction with Hi-GAL 70 to $500 \mu \mathrm{m}$ images, MSX emission maps at $8,12,14$, and $21 \mu \mathrm{m}$, and WISE 12 and $24 \mu \mathrm{m}$ images, and fit the resulting SEDs to derive $T_{\text {dust }}$ and $L$. Each SED was fit with either one (greybody-only) or two (greybody+blackbody) components, depending one whether the source was best represented by a single cold component or a combination of cold and hot components. Clumps were fit with two components if they had at least two flux measurements at $\lambda<70 \mu \mathrm{m}$. All twelve of our sources are represented in the Urquhart et al. (2018) sample, and the median ratio of their luminosities to ours is +1.5 . The Urquhart et al. (2018) $L$ are higher than our greybody-derived $L$ in all cases; this likely reflects the fact that our greybody fits are exclusively single- 
component fits, while the fitting procedure of Urquhart et al. (2018) requires that at least eleven of our sources were fit with two components in their analysis. The explicit inclusion of a hot component in the fit would be expected to increase the overall luminosity derived for a clump.

\subsection{SED Modeling}

In $\S 4.2 .1$, 4.2.2, and 4.2.3, we provide brief summaries of the model assumptions and underlying physics for each of the three SED model types we used (Robitaille et al. 2006, Robitaille 2017; Zhang \& Tan 2018). While we present the Robitaille (2017) results first in our figures for easy visual comparison (Figure 5, and all figures in Appendix A), we have chosen to present the models in chronological order by publication date in these summary sections, as the improvements in the Robitaille (2017) models were directly influenced by the Robitaille et al. (2006) models.

In Figure 5 and Appendix A, the $\chi^{2}$ value shown on the plots is $\chi^{2}$ per data point, where a "data point" is defined as any flux density used for the fit that is not an upper or lower limit. (For G11.92, for example, $n_{\text {data }}$ is 6.) From this point forward, any discussion of " $\chi$ " values refers to $\chi^{2}$ per data point unless explicitly stated otherwise.

\subsubsection{Robitaille et al. (2006) Model Grid}

The Robitaille et al. (2006) models are a single grid of 200,000 model SEDs for YSOs. All Robitaille et al. (2006) models include a central star, rotationally-flattened infalling (Ulrich-type) envelope, bipolar cavities, and a flared accretion disk. The models use the dust optical constants of Laor \& Draine (1993), and neither include emission from PAHs nor account for the possibility of ice-coated grains. The models were interpolated along evolutionary tracks in order to derive stellar radius $\left(R_{\star}\right)$ and stellar temperature $\left(T_{\star}\right)$ from a given combination of stellar mass $\left(\mathrm{M}_{\star}\right)$ and age $\left(t_{\star}\right)$. They use two sets of evolutionary tracks: Bernasconi \& Maeder (1996) for stars with $\mathrm{M}_{\star}>9 M_{\odot}$, Siess et al. (2000) for stars with $\mathrm{M}_{\star}$ $<7 M_{\odot}$, and a combination of the two for stars with $7 M_{\odot}<$ $\mathbf{M}_{\star}<9 M_{\odot}$. The grid values of $\mathbf{M}_{\star}$ and $t_{\star}$ were sampled from probability density distributions: $\mathbf{M}_{\star}$ was sampled between $\mathrm{M}_{\min }=0.1 M_{\odot}$ and $\mathrm{M}_{\max }=50 M_{\odot}$ such that there was a constant density of models in $\log _{10} \mathrm{M}_{\star}$ space, and $t_{\star}$ was sampled between $\mathrm{t}_{\min }=10^{3} \mathrm{yr}$ and $\mathrm{t}_{\max }=10^{7} \mathrm{yr}$ such that there was a nearly-constant density of models in $\log _{10} t_{\star}$ space, with a slight bias toward higher values of $t_{\star}$.

The ranges of the envelope accretion rate $\dot{M}_{e n v} / M_{\star}$, envelope outer radius, cavity opening angle $\theta_{\text {cavity }}$, and cavity density are dependent on the age of the central source. Overall in the grid, $\dot{M}_{e n v} / M_{\star}$ varies from $\sim 5 \times 10^{-4}$ to $\sim 10^{-9}$, and spans two orders of magnitude for any given source age. $\dot{M}_{e n v} / M_{\star}$ is sampled uniformly in logarithmic space. It is held constant for $t_{\star}<10^{4} \mathrm{yr}$, then decreases, and finally goes to zero around $10^{6}$ yr. For models with $M_{\star}>20 M_{\odot}, \dot{M}_{e n v} / M_{\star}$ was sampled with the same range as a $20 M_{\odot}$ model. That is, $\dot{M}_{e n v} / M_{\star}$ for sources with $M_{\star}>20 M_{\odot}$ is no longer specific to each stellar mass, but a general rate used for all stars with $M_{\star}>20 M_{\odot}$.

The envelope outer radius ranges from $10^{3}$ to $10^{5} \mathrm{AU}$, sampled uniformly in $\log (R)$ space. Bipolar cavities follow a conical shape described in cylindrical coordinates by $z=c \omega^{d}, \omega$ is the radial coordinate, $d=1.5$ is a fixed value, and $c$ is a constant of proportionality defined as $c=$ $\left(R_{e n v}^{m a x}\right) /\left(R_{e n v}^{\max } \tan \left(\theta_{\text {cavity }}\right)\right) . \quad \theta_{\text {cavity }}$ is sampled from a range of values that increases with $t_{\star}$; values range from $0^{\circ}$ to $60^{\circ}$ in the grid overall, but are limited to $\sim 0^{\circ}-10^{\circ}$ for the youngest sources and gradually shift to $\sim 20^{\circ}-60^{\circ}$ for the oldest sources. Envelope cavity density is sampled from a range one order of magnitude wide that decreases with evolutionary age; it ranges from $8 \times 10^{-20} \mathrm{~g} \mathrm{~cm}^{-3}$ to $1 \times 10^{-22} \mathrm{~g} \mathrm{~cm}^{-3}$, except in cases where the ambient density is greater than the cavity density. In such cases, the cavity density is reset to the density of the ambient medium, which is constant in both space and time and ranges from $\sim 1.67 \times 10^{-22}\left(M_{\star} / M_{\odot}\right) \mathrm{g} \mathrm{cm}^{-3}$ to $\sim 6.68 \times 10^{-22}\left(M_{\star} / M_{\odot}\right) \mathrm{g} \mathrm{cm}^{-3}$ (for precise ranges and sampling conditions, see $\S 2.2 .2 .5$ of Robitaille et al. 2006).

The flared accretion disk is described by five parameters: disk mass, disk outer radius, disk inner radius, disk structure, and disk accretion rate. Disk mass is originally sampled from $\sim 0.001-0.1 M_{\odot}$ for sources with $t_{\star}<1 \mathrm{Myr}$, and then sampled over a wider range of masses for later evolutionary stages. Disk outer radius is usually associated with the centrifugal radius $\mathrm{R}_{C}$, so $\mathrm{R}_{C}$ is sampled from 1 to $10,000 \mathrm{AU}$, but is time-dependent such that earlier evolutionary stages may have smaller radii than later stages. Disk inner radius was set to the dust sublimation radius $\mathrm{R}_{\text {sub }}$ for one third of the models, and sampled between $\mathrm{R}_{\text {sub }}$ and $100 \mathrm{AU}$ or the disk outer radius, whichever was smaller, in the remaining two thirds of the models. Additionally, the envelope inner radius was set to the disk inner radius for all models. The disk structure is described by the disk flaring parameter $\beta$ and scale height factor $\mathrm{z}_{\text {factor }}$, both of which were sampled from ranges dependent on the disk outer radius. The disk accretion rate is calculated using the disk $\alpha_{\text {disk }}$ parameter (a unitless measure of the efficiency of angular momentum transport, which is dependent on disk radius and always less than 1) and accretion-to- $\alpha$ relations published in prior works (for details, see Robitaille et al. 2006, and references therein). $\alpha_{\text {disk }}$ is sampled from $10^{-3}$ to $10^{-1}$ in log space.

\subsubsection{Robitaille (2017) Model Sets}

The Robitaille (2017) models are a set of eighteen different YSO model grids, each with 10,000 to 80,000 models. The models were created using a similar computational method to Robitaille et al. (2006), but with different physical components and parameter ranges. Each grid (referred to as a "model set" in Robitaille (2017), nomenclature that we henceforth adopt here) contains a different combination of physical components. Each model set includes a central stellar/protosteller source, and then may or may not include 1) an ambient medium, 2) a power-law envelope or an Ulrichtype envelope, 3) bipolar cavities, 4) a passive disk, and 5) an "inner hole" (gap between the stellar surface and the inner radius of the disk/envelope). The Robitaille (2017) models do not assume a particular evolutionary track; it is left to the user to determine additional protostellar properties (other than those returned by the model) using the evolutionary track of their choice. Users are encouraged to test multiple model sets against their data and identify trends in model results in order to determine which physical components do or do not make a significant difference to the goodness-of-fit.

Given that we already have evidence that our targets have outflow activity (e.g. Cyganowski et al. 2008, 2009) and most are embedded in IRDCs, we chose to run all models with bipolar cavities, except for two controls (one with a disk, envelope, and no cavities and one with an envelope and neither a disk nor cavities). However, while the outflow activity of 
19
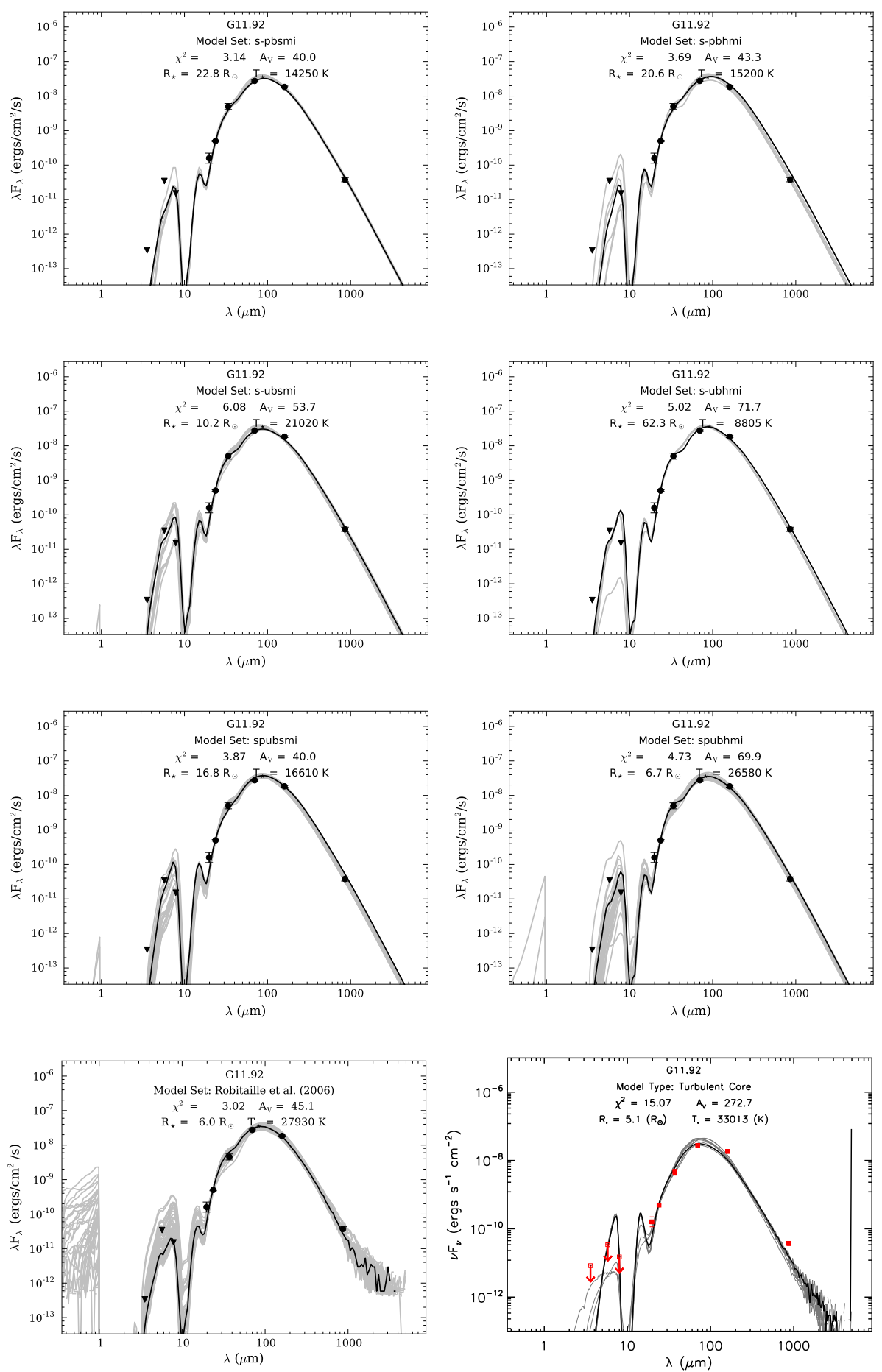

FIG. 5.- Robitaille (2017), Robitaille et al. (2006), and Chang \& Tan (2018) SED modeling results for G11.92-0.61. The top three rows are the best six model sets from the Robitaille (2017) model package, the bottom left panel shows the results from Robitaille et al. (2006), and the bottom right panel shows the results from Chang \& Tan 2018). All $\chi^{2}$ values shown are $\chi^{2} / \mathrm{n}_{\text {data }}$, where $\mathrm{n}_{\text {data }}$ is the number of data points used for the fitting that are not upper or lower limits. For this source, $\mathrm{n}_{\text {data }}=6$. The best-fit model for each model set is denoted by a black line. The gray lines are SED models whose $\chi^{2}$ per data point values were within a factor of 5 of the best-fit $\chi^{2}$ per data point. The spike in the last wavelength bin in the Zhang \& Tan (2018) model is due to a binning error at the first and last wavelength bins: all flux above or below the longest or shortest wavelength is binned into the last or first wavelength bin. The error is present for all Bhang
$\&$ Tan (2018) models, but only produces a significant spike in a few. 
our target sources is suggestive of the presence of disks, they have been confirmed in few of our targets (e.g. G11.92-0.61). Therefore, we have chosen to run all models with bipolar cavities - including those without disks - in order to avoid biasing our analysis towards only models with disks.

In all eighteen Robitaille (2017) model sets, $\mathrm{T}_{\text {star }}$ varies from $2000 \mathrm{~K}$ to $30000 \mathrm{~K}$. In models with a disk, the disk shape varies from from hydrostatic (flared) to flat. All disks in all models are passive (accretion is not explicitly included in the model). A detailed explanation of the reasoning for using only passive disks can be found in Robitaille (2017), § 3.2.2. The disk density distribution goes as $\mathrm{r}^{(\beta-p)} \mathrm{e}^{(z / h)^{2}}$, where disk flaring power $(1<\beta<1.3)$, disk surface density power $(-2$ $<p<0)$, and disk scale height ( $1 \mathrm{AU}<h<20 \mathrm{AU})$ are free parameters. The envelope can be either Ulrich-type, in which case the centrifugal radius $\left(\mathrm{R}_{C}\right)$ varies from 50 to $5000 \mathrm{AU}$, or power-law, in which case the envelope power $(\gamma)$ ranges from -2 to -1 . The shape of the bipolar cavities follows a power-law, where the cavity power $(c)$ varies from 1 to 2 . The bipolar cavities are assumed to be filled with dust of a constant density, where the density ranges from $10^{-23}$ to $10^{-20} \mathrm{~g} \mathrm{~cm}^{-3}$. The ambient medium is defined as a lower limit to the density and temperature of the envelope $\left(\mathrm{T}_{a m b}=10 \mathrm{~K}, \rho_{a m b}=10^{-23} \mathrm{~g}\right.$ $\mathrm{cm}^{-3}$ ). The dust in the Robitaille (2017) models is taken from Draine (2003a b) and Weingartner \& Draine (2001), and does not include emission from PAHs. Each SED is computed for nine viewing angles between $0^{\circ}$ and $90^{\circ}$, where the viewing angles are selected using stratified sampling: viewing angle is randomly chosen within a specific range, so that each SED is sampled at one random angle between $0^{\circ}$ and $10^{\circ}$, one random angle between $10^{\circ}$ and $20^{\circ}$, and so on up to $90^{\circ}$.

The model sets we used are as follows:

s-pbhmi: - model contains a central star, no passive disk, a power-law envelope, a bipolar cavity, an ambient medium, and a variable inner envelope radius (rather than the inner radius being set to the dust sublimation radius). This variability has the effect of creating an "inner hole" between the inner radius of the envelope and the stellar surface.

s-ubhmi: - model contains a central star, no passive disk, an Ulrich envelope, a bipolar cavity, an ambient medium, and an inner hole.

s-pbsmi: - model contains a central star, no passive disk, a power-law envelope (no rotational flattening), a bipolar cavity, an ambient medium, and no inner hole (i.e. the inner radius is the dust sublimation radius).

s-ubsmi: - model contains a central star, no passive disk, an Ulrich (rotationally-flattened) envelope, a bipolar cavity, an ambient medium, and no inner hole.

spubsmi: - model contains a central star, a passive disk, an Ulrich envelope, a bipolar cavity, an ambient medium, and no inner hole.

spubhmi: - model contains a central star, a passive disk, an Ulrich envelope, a bipolar cavity, an ambient medium, and an inner hole.

spu-smi: - model contains a central star, a passive disk, an Ulrich envelope, no bipolar cavities, an ambient medium, and no inner hole. $s$ - $u$-smi: - model contains a central star, no passive disk, an Ulrich envelope, no bipolar cavities, an ambient medium, and no inner hole.

A complete key, including diagrams, for all eight of these model sets can be found in Robitaille (2017), Table 2.

\subsubsection{Zhang \& Tan (2018) Model Grid}

The Zhang \& Tan (2018) models are a grid of $~ 9000$ YSO model SEDs. These models are based on the Turbulent Core theory of high-mass star formation (McKee \& Tan 2003). The Zhang \& Tan models all assume a central source, disk, envelope, and bipolar outflow; they neither assume nor fit an ambient medium or emission from the parent clump. The model grid is composed of five variables (three physical, two observational): core mass, mass surface density, stellar mass, $\mathrm{A}_{V}$ along the line of sight, and inclination/viewing angle. Core mass is sampled from 10 to $480 M_{\odot}$, mass surface density ranges from $0.1 \mathrm{~g} \mathrm{~cm}^{-3}$ to $3.16 \mathrm{~g} \mathrm{~cm}^{-3}$, and stellar mass ranges from 0.5 to $160 M_{\odot}$. Each model SED is sampled at 20 viewing inclinations, from $\cos \left(\theta_{\text {view }}\right)=0.975$ to $\cos \left(\theta_{\text {view }}\right)=0.025$. The range of $\mathrm{A}_{V}$ is set by the user; we chose to use $40<\mathrm{A}_{V}$ $<1000$, as we did for both types of Robitaille models.

In the Zhang \& Tan (2018) models, the initial core is assumed to have an $\mathrm{r}^{-3 / 2}$ power-law density distribution, and is assumed to exhibit inside-out collapse and rotationalflattening (i.e. is assumed to be an Ulrich-type envelope). Zhang \& Tan (2018) assume that the ratio of disk mass to protostellar mass is constant, at $M_{\text {disk }} / M_{\star}=1 / 3$. They assume that all disks are hydrostatic (modified alpha disks). In order to return protostellar radius, temperature, and luminosity, the models assume the evolutionary tracks of Hosokawa \& Omukai (2009). Zhang \& Tan (2018) use the same dust models as Robitaille et al. (2006).

\subsection{Model Results: Robitaille et al. (2006), Robitaille (2017), and Zhang \& Tan (2018)}

Due to the different physical assumptions and parameters fit by each model, the number of physical parameters that could be compared directly is small. Table 9 shows the stellar radii $\left(R_{\star}\right)$ and stellar temperatures $\left(T_{\star}\right)$ returned by each of the three model grids, as well as the Stefan-Boltzmann luminosities $\left(4 \pi R_{\star}^{2} \sigma T_{\star}^{4}\right)$ calculated from those radii and temperatures. The $\chi^{2}$ values shown are $\chi^{2}$ per data point, as described in $\S 4.2$ The individual $R_{\star}$ and $T_{\star}$ values returned by the three different sets of SED models for a particular source frequently span up to two orders of magnitude. However, the Stefan-Boltzmann luminosities calculated from the different combinations of $R_{\star}$ and $T_{\star}$ (hereafter $L_{\star}$ ) tend to agree to within a factor of 3 .

Robitaille (2017) stress that the model sets therein are best used to compare how much the presence or absence of a particular physical component (e.g. bipolar cavities) affects the accuracy of each model. In order to accomplish this, the author suggests that a Bayesian analysis (rather than $\chi^{2}$ scores alone) are needed. Unfortunately, we cannot compare Bayesian scores from the Robitaille (2017) models to Bayesian scores from the Robitaille et al. (2006) and Zhang \& Tan (2018) set, as this approach of comparing probabilities assumes the models in question have similar underlying parameters and parameter ranges. The Robitaille et al. (2006) and Zhang \& Tan (2018) models sample different parameter ranges and, in the case of Zhang \& Tan (2018), different parameters altogether, so the comparison of probabilities cannot 
be performed. For the purposes of comparing the results from the different sets of published models, we use $\chi^{2}$. A detailed discussion of the Bayesian scores for the Robitaille (2017) models - and how this approach affects the overall trends as compared to the $\chi^{2}$ analysis - can be found in Appendix B.

\subsubsection{Expected Luminosity Sensitivity}

The only source which had no $37.1 \mu \mathrm{m}$ emission detected toward the EGO is G10.29-0.13 (see Table 3, Fig. 7). This source is also a non-detection at 19.7 and $24 \mu \mathrm{m}$, and has fairly isolated Hi-GAL and ATLASGAL emission compared to the rest of the sample. Therefore, based on the non-detection of this source and its comparative isolation and morphological simplicity, we use G10.29-0.13 as a test case in order to estimate the minimum luminosity sensitivity of the SOFIA observations. The $37.1 \mu \mathrm{m}$ observations of 10.29-0.13 have an integration time of $502 \mathrm{~s}$ and $\sigma=0.26$ $\mathrm{Jy} \mathrm{beam}^{-1}$, and the assumed distance is $1.9 \mathrm{kpc}$.

We construct a synthetic SED for this source by inserting the $3 \sigma$ upper limits at 37.1 and $24 \mu \mathrm{m}$ as actual photometric measurements with uncertainties of $1 \sigma$. The $19.7 \mu \mathrm{m}$ data remain as upper limits, while the values and treatment of flux densities from the other six wavelengths are likewise unchanged. We modeled this SED using the eight Robitaille (2017) model sets described in $\S 4.2 .2$, and the bestfit model returned a Stefan-Boltzmann luminosity of $L_{\min }=$ $1.1 \times 10^{3} L_{\odot}$.

This approximate lower limit is consistent with $92 \%$ of the luminosities shown in Table 9 There are four models which produced $L_{\star}<1.1 \times 10^{3}$, and interestingly all of these come from the Zhang \& Tan (2018) models. These four low luminosity Zhang \& Tan (2018) model results also correspond to the largest discrepancies between the three types of models assessed. See additional discussion of the trends, limitations, and overall quality of the three model packages below.

\subsubsection{Stefan-Boltzmann Luminosities}

The $L_{\star}$ (calculated from $R_{\star}$ and $T_{\star}$ ) are almost always larger than the $L$ returned by the greybody fits, typically by a factor of $\sim 2$. This trend is consistent with the fact that our greybody fits are single-component and largely account for emission from cold dust, whereas the fits to the full SEDs can also account for emission from hotter components (e.g., hot cores) that emit predominantly in the NIR and MIR. This is also consistent with our previous comparison to the luminosities reported in Urquhart et al. (2018). We find that the ratio between the Urquhart et al. (2018) luminosities and ours has decreased; the median ratio between the Urquhart et al. (2018) $L$ and those listed in Table 9 is now +1.27 . The median ratios between the Urquhart et al. (2018) $L$ and ours for the individual model packages are +1.22 for Robitaille et al. (2006),+1.31 for Robitaille (2017), and -0.21 for Zhang \& $\operatorname{Tan}(2018)$.

There are a few cases in which the $L_{\star}$ calculated from our SED model results was lower than $L$ returned by our greybody fits (hereafter $L_{\text {grey }}$, to distinguish from $L_{\star}$ ). We believe that these cases can be explained by flux or confusion limitations during the aperture photometry procedure, which then lead to the SED models returning low $L_{\star}$ results. We identify three categories of SEDs which exhibit the $L_{\text {grey }}>L_{\star}$ discrepancy:

Confusion problems at $160 \mu \mathrm{m}:-$ The majority of $L_{\star}-L_{\text {grey }}$ discrepancies occur in sources which suffer from angular confusion in the $160 \mu \mathrm{m}$ Hi-GAL data (G12.91-0.03, G18.89-0.47,
G22.04+0.22; see Figs. 10, 14, and 16. In all cases, the measured $160 \mu \mathrm{m}$ flux was unexpectedly high, not low. For these three sources, at least one and as many as all three SED modeling packages produced $L_{\star}<L_{\text {grey }}$. While we tried to account for the $160 \mu \mathrm{m}$ confusion issue by significantly increasing the errors on the flux measurements for these sources, it is still possible that either a) the high $160 \mu \mathrm{m}$ values or the large uncertainties on those values are leading to poor fits from the SED modeling packages, or b) the high $160 \mu \mathrm{m}$ points lead to greybody fits that overestimate $L_{\text {grey }}$. Either cause (or possibly both) would result in $L_{\text {grey }}>L_{\star}$.

Poorly-constrained SEDs:- All sources use flux measurements at eight or nine separate wavelengths in order to construct the SEDs. In most sources, three of these data points (IRAC bands) are always upper limits. However, in one source (G10.29-0.13), six of the nine flux measurements $(67 \%)$ are upper limits, and the Zhang \& Tan (2018) SEDderived $L_{\star}$ value is extremely low. We believe that this discrepancy can be explained by the very poor constraints on the MIR and NIR flux measurements, which makes it possible to fit a wide variety of models to the data (see Fig. 7); it is therefore unsurprising that at least one of these models produces a very low $L_{\star}$.

Upper limits at $19 \mu \mathrm{m}:-$ There are four sources in our sample which are non-detections at $19 \mu \mathrm{m}$, and so use upper limits for the $19 \mu \mathrm{m}$ flux instead of direct measurements. Of these four sources, two have additional issues (overall poor constrains on the SED, confusion problems at $160 \mu \mathrm{m}$ ) that have already been discussed. However, the remaining two sources have no additional issues with flux measurements, but do still have $L_{\star}<L_{\text {grey }}$ for at least one SED-derived $L_{\star}$. It is possible that, in these cases, the use of an upper limit at $19 \mu \mathrm{m}$ is allowing the SED modeling packages to underestimate the true $19 \mu \mathrm{m}$ fluxes, which then leads to spuriously low $L_{\star}$ values. See Figs. 8 and 12 for SEDs for these two sources.

\subsubsection{Spread in Physical Parameters Returned by the Radiative-Transfer Models}

Within our sample, the values of $R_{\star}, T_{\star}$, and $L_{\star}$ produced by a given model package typically span one order of magnitude. Exceptions are the $R_{\star}$ values produced by the Robitaille et al. (2006) models, which span two orders of magnitude across our 12 sources, and the $L_{\star}$ values from the Zhang \& Tan (2018) models, which span four orders of magnitude. Conversely, when comparing the results of all three model packages for a given source, the $R_{\star}$ results typically span one order of magnitude, but can span two; the $T_{\star}$ results typically also span one order of magnitude. The $L_{\star}$ results are more consistent with each other: $75 \%$ of the $L_{\star}$ results from all three models agree to within a factor of 3 . This result should not be overlooked - the models rarely converge for physical parameters that assume specific geometries (i.e. $R_{\star}$ ), but do converge to properties that can be extracted from SED shape alone (i.e. $L_{\star}$ ).

The different model packages will have difficulty converging to a single combination of $R_{\star}$ and $T_{\star}$ if the geometry of a single source is not one of those assumed by the models (e.g. multiple protostellar sources, accretion disk much more or less massive than the range in the model grid, etc.). All three of these sets of models do fit only one protostar at a time - they assume only one source is contributing to the emission. However, recent research suggests that less-massive protostars may form in the accretion reservoirs of more massive 
TABLE 9

Stellar Radius, TEMPERATURE, AND LUMINOSITy RESUlts FOR SED MODEL GRIDS

\begin{tabular}{|c|c|c|c|c|c|c|c|c|c|c|c|c|c|}
\hline \multirow{2}{*}{$\begin{array}{l}\text { Source } \\
\text { Name }\end{array}$} & \multicolumn{5}{|c|}{ Robitaille $(2017)^{a, b}$} & \multicolumn{4}{|c|}{ Robitaille et al. $(2006)^{a}$} & \multicolumn{4}{|c|}{ Zhang \& Tan $(2018)^{a}$} \\
\hline & Model & $R_{\star}\left(R_{\odot}\right)$ & $T_{\star}(\mathrm{K})$ & $L_{\star}\left(10^{3} L_{\odot}\right)$ & $\chi^{2}$ & $R_{\star}\left(R_{\odot}\right)$ & $T_{\star}(\mathrm{K})$ & $L_{\star}\left(10^{3} L_{\odot}\right)$ & $\chi^{2}$ & $R_{\star}\left(R_{\odot}\right)$ & $T_{\star}(\mathrm{K})$ & $L_{\star}\left(10^{3} L_{\odot}\right)$ & $\chi^{2}$ \\
\hline G10.29-0.13 & spubhmi & 49.1 & 6640 & 4.15 & 0.0003 & 71.0 & 7405 & 13.41 & 0.002 & 7.3 & 4994 & 0.03 & 0.001 \\
\hline G10.34-0.14 & s-pbhmi & 27.5 & 7980 & 2.71 & 1.51 & 79.3 & 4492 & 2.27 & 1.45 & 21.7 & 6835 & 0.91 & 3.98 \\
\hline G11.92-0.61 & s-pbsmi & 22.8 & 14250 & 18.97 & 3.14 & 6.0 & 27930 & 19.39 & 3.02 & 5.1 & 33013 & 27.34 & 15.07 \\
\hline G12.91-0.03 & s-pbhmi & 6.4 & 19940 & 5.73 & 1.51 & 118.8 & 4355 & 4.49 & 2.48 & 11.2 & 16298 & 7.83 & 20.43 \\
\hline G14.33-0.64 & s-pbsmi & 31.4 & 7976 & 3.53 & 0.45 & 110.7 & 4428 & 4.17 & 0.81 & 11.2 & 16298 & 7.83 & 1.89 \\
\hline G14.63-0.58 & s-pbhmi & 11.8 & 11330 & 2.03 & 3.68 & 68.6 & 4172 & 1.26 & 1.94 & 18.5 & 6780 & 0.64 & 20.54 \\
\hline G16.59-0.05 & s-pbhmi & 31.8 & 11020 & 13.20 & 1.12 & 65.0 & 7510 & 11.89 & 0.53 & 5.1 & 33013 & 27.34 & 11.84 \\
\hline G18.89-0.47 & s-pbhmi & 14.6 & 10610 & 2.39 & 1.00 & 96.7 & 4234 & 2.66 & 4.2 & 7.3 & 4994 & 0.03 & 37.73 \\
\hline G19.36-0.03 & s-pbsmi & 8.4 & 14420 & 2.70 & 1.73 & 98.3 & 4493 & 3.48 & 1.41 & 13.6 & 14585 & 7.41 & 10.93 \\
\hline $\mathrm{G} 22.04+0.22$ & s-pbhmi & 14.3 & 12570 & 4.52 & 1.81 & 27.3 & 9345 & 5.03 & 1.92 & 11.2 & 16298 & 7.83 & 7.66 \\
\hline G28.83-0.25 & s-pbhmi & 74.3 & 8766 & 28.85 & 0.81 & 293.4 & 4355 & 27.40 & 1.62 & 42.9 & 12552 & 40.43 & 12.96 \\
\hline $\mathrm{G} 35.03+0.35$ & spubhmi & 69.9 & 8552 & 23.13 & 1.08 & 4.8 & 31560 & 20.23 & 1.31 & 5.1 & 33013 & 27.34 & 0.66 \\
\hline $\operatorname{Median}^{c}:$ & $\cdots$ & 25 & 10800 & 4.3 & 1.31 & 75 & 5950 & 4.8 & 1.54 & 11 & 15440 & 7.8 & 12.40 \\
\hline
\end{tabular}

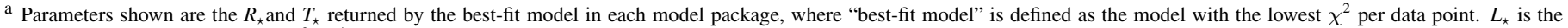
Stefan-Boltzmann luminosity $\left(4 \pi \mathrm{R}_{\star}^{2} \sigma \mathrm{T}_{\star}^{4}\right)$ calculated from each combination of $R_{\star}$ and $T_{\star}$

b Since the Robitaille 2017) model package contains multiple model grids, we include an extra column stating the name of the model set to which the best-fit model belongs.

${ }^{\mathrm{c}}$ Median values are computed for our twelve sources for a given parameter within a given model package. The median $L_{\star}$ of all 36 best-fit models is $5.83 \times 10^{3} L_{\odot}$.

companions $(<0.2 \mathrm{pc}$ separation), and in fact there is compelling evidence that this is the case for at least one of our sources (G11.92-0.61; see Cyganowski et al. 2017, and references therein). Furthermore, the nine different wavelengths used to create these SEDs probe different spatial scales due to the angular resolution of individual telescopes (e.g. 1."66 for Spitzer IRAC band I1 versus 19." 2 for the ATLASGAL survey). This may also contribute to the scatter in $R_{\star}$ and $T_{\star}$ for individual sources if, for instance, the NIR and MIR fluxes are correctly attributed to only one protostellar source but the FIR fluxes are instead the blended fluxes of multiple adjacent sources in a clustered environment.

Finally, it is possible that the SED fits themselves are good but the fits to individual parameters poor because the assumption by the models of hierarchical structure (i.e. central source(s), disk, envelope, cavities) is true, but the assumption that all structures are present in a single source (i.e. the same individual protostar) is false. Possible scenarios in which this could occur are the case in which multiple cores are present within a single envelope, such as for a protobinary system, or IR-bright outflows due to multiple cores, even if only one core is visible in the MIR.

\subsubsection{Trends by Model}

The trends in $R_{\star}, T_{\star}$, and $L_{\star}$ suggest that the Robitaille et al. (2006) models favor cooler, larger - and hence younger - protostars to describe our data, while the Zhang \& Tan (2018) models favor smaller, hotter protostars. The Robitaille (2017) $R_{\star}$ and $T_{\star}$ results typically fall between the two other model packages, but the resulting Stefan-Boltzmann luminosities agree fairly well with those of Robitaille et al. (2006). The Robitaille et al. (2006) models tend to produce similar $\chi^{2}$ values as the $\chi^{2}$ of the best-fit Robitaille (2017) models, and produce $\chi^{2}$ values that are lower than those of the Zhang \& Tan (2018) models in all but two cases. The only notable trend in the Robitaille et al. (2006) models is a slight tendency to overestimate the $37.1 \mu \mathrm{m}$ flux (see Figs. 7 through 18, Appendix A). This effect is sometimes also present in the Robitaille (2017) models, though to a lesser degree.

We found that the Robitaille (2017) models without bipolar cavities routinely gave very poor results, as expected ( $\chi^{2}$ val- ues that are factors of $\sim 10$ to 40 higher than the models with bipolar cavities).

Models s-pbsmi through spubhmi are shown Figures 7 through 18 in Appendix A; models spu-smi and s-u-smi are not shown, as in all cases they produced significantly poorer fits than any of the other six Robitaille (2017) model sets. Overall, the best-fit models (as determined by minimum $\chi^{2}$ value) were always of the sets s-pbhmi, s-pbsmi, or spubhmi, in order of decreasing frequency. That is, the models overall favored no disk and a power-law envelope. For some sources, some model sets are clearly inappropriate, as they consistently underestimate long-wavelength emission or overestimate short-wavelength emission (e.g. model sets s-ubsmi and s-ubhmi for G14.33-0.64, Figure 11, model sets s-ubsmi, subhmi, spubsmi, spubhmi for G14.63-0.58, Figure 12). However, in very few cases were there no Robitaille (2017) model sets that could reasonably fit our data.

In general, we find that the Zhang \& Tan (2018) models fit some of the mid-IR fluxes fairly well, but consistently overestimate the $37 \mu \mathrm{m}$ and $70 \mu \mathrm{m}$ fluxes and underestimate the emission at $160 \mu \mathrm{m}$ and $870 \mu \mathrm{m}$. The sources for which this is not the case are either very poorly constrained (G10.29) or contain known UC H II regions (G35.03). The Zhang \& Tan (2018) models also have the highest $\chi^{2}$ value in all but these two sources and, in these two sources, $\chi^{2}<1$, which indicates that those models may be overfit. In general, the Zhang \& Tan (2018) $\chi^{2}$ values are an order of magnitude higher than at least one of the other two models; in nine of twelve sources, it is higher than both. Since the Zhang \& Tan (2018) models do not include an ambient medium/emission from the parent clump, it is likely that this omission is leading the model to underestimate our measured emission at long wavelengths, and to produce higher $\chi^{2}$ values. The cause of the overestimation in the MIR is currently unclear, though this trend is also present to some degree in the other two model packages as well. Combined with the underestimation of the FIR emission, this MIR overestimation creates a trend wherein the Zhang \& Tan (2018) models in general seem to be pushed toward SEDs with peaks at slightly shorter wavelengths than our data exhibit; this may indicate that the Zhang \& Tan (2018) models tend to produce better results for slightly 
older or less deeply-embedded sources.

De Buizer et al. (2017) tested the Zhang \& Tan (2018) models against data from their SOFIA Massive Star Formation Survey (SOMA), and compared their results to results from the Robitaille et al. (2006) models. In general, the Zhang \& Tan (2018) models produced good results for their sample, and they do not note a systematic underestimation of long-wavelength emission from these models. However, the SOMA survey identifies four source types, and De Buizer et al. (2017) examines only their Type II ("Hypercompact") sources. De Buizer et al. (2017) state that these sources often have jet-like radio emission, and MIR emission that extends beyond the radio emission. While the second criterion applies to our sample, the first does not. The characteristics of our sources are a better match to their Type I ("MIR Sources in IRDCs," which is a quality nearly all of our sources share) or their Type IV ("Clustered Sources," which recent work (e.g. Cyganowski et al. 2017) shows is the case for at least one source in our sample, and likely more). De Buizer et al. (2017) note that there is a rough evolutionary sequence from Type I to Type III sources. If this sequence is accurate, and our sources are more similar to their Type I sources, then our sample would be slightly younger than that evaluated in De Buizer et al. (2017). In this case, the MIR-emitting sources are indeed likely to be cooler and/or more deeply embedded than those in the SOMA Type II sample, and this would at least partially explain the discrepancy between the model results for our sample (i.e. consistent underestimation of FIR emission) and theirs.

\subsection{Do the Robitaille (2017) Model Sets Tell Us Something About Source Structure?}

The Robitaille (2017) model sets are the only models evaluated in this work which explicitly allow the user to test multiple different source geometries. We briefly evaluate the overall trends in the geometries of the best-fit model sets for our sources in order assess what, if anything, the Robitaille (2017) models are telling us about the structure of the protostellar sources in our sample. Model results for each individual source can be found in Appendix B, along with a discussion of how the method of evaluating which is the "best" fit affects these trends.

G10.29-0.13 is excluded from this discussion of general trends, as its SED is very poorly constrained. For the remaining 11 sources, the Robitaille (2017) model package frequently returns best-fit models which have power-law envelopes and no disks, and do not favor either the presence or absence of an inner hole. However, among model sets specifically with no disk and with a power-law envelope, there is a clear preference $(64 \%$ to $36 \%$, or 7 to 4 ) for models with an inner hole. That is, a power-law envelope favors a larger distance between the inner edge of the envelope and the stellar surface. Among models with no disk and an Ulrich envelope, this trend is exactly reversed, with $64 \%$ of models ( 7 out of 11) preferring no inner hole and only $36 \%$ (4 out of 11) having one; in this case, the addition of rotation seems to favor a smaller distance between the envelope inner radius and the stellar surface. It should also be noted that only three of our eight model sets contain a disk (spubhmi, spubsmi, spusmi), and one of these (spu-smi) was expected to generally give poor fits to our data anyway due to its lack of bipolar cavities. It is possible that the bias against disks may be due, at least in part, to the relative dearth of individual model sets with disks compared to those without. Of the eighteen model sets available to us, we chose to run the six models with bipolar cavities and to "control" models. Two of the six model sets with bipolar cavities have disks, while four do not - this 2-to-1 ratio is simply a feature of the model sets available to us. However, this ratio may give an unphysical "advantage" to the disk-lacking models in the evaluation of model-set statistics.

The lack of disks in the favored models is inconsistent with our more detailed knowledge of particular sources, such as G11.92-0.61 (Ilee et al. 2016, 2018) and G16.59-0.05 (Moscadelli et al. 2016; Rosero et al.|2016), as well as our more general knowledge of these sources based on their additional attributes (e.g. shocked $\mathrm{H}_{2}$ emission (Cyganowski et al. 2008), both Class I and Class $\mathrm{II} \mathrm{CH}_{3} \mathrm{OH}$ masers (Cyganowski et al. 2009), etc.). For most sources, the Robitaille (2017) models with disks do not appear (visually) to be significantly different in the mid-infrared ( $\sim 10$ to $40 \mu \mathrm{m}$; see Figures 7 through 18) from models without them. This region of the SED is frequently dominated by hot dust emission from the outflow cavity and heated portions of the envelope. A disk that is small relative to the mass of the protostar, or highly extincted by embedding material, might manifest its presence less strongly in the mid-infrared portion of the SED, in which case SED modeling would not need to invoke a disk in order to reproduce the given data.

\section{5. $L / M$ and Evolutionary State}

Figure 6 shows the luminosity-to-mass ratio $L / M$ versus mass $M$ for each source using each of the four derived luminosities. For both $L / M$ and $M, M$ is the average of the $\mathrm{NH}_{3}$-derived and greybody-derived masses for each source. The errors on $L / M$ are determined from the error propagation equation and the errors on $L$ and $M$, respectively. The uncertainty on each $L$ value, $\sigma_{L}$, is the median absolute deviation from the median (MAD) of the luminosities of all fits with $\chi^{2}$ within a factor of 3 of the best-fit $\chi^{2}$ value. The uncertainty on each $M$ value, $\sigma_{M}$, is calculated from the uncertainties on both the greybody-derived and $\mathrm{NH}_{3}$-derived temperatures, the $870 \mu \mathrm{m}$ flux $\mathrm{F}_{870 \mu \mathrm{m}}$, and distance, $\mathrm{D}$, using the error propagation equation. Uncertainties in temperature are reported in Table 8, and uncertainties in $\mathrm{F}_{870 \mu m}$ are reported in Table 7 Distance uncertainties for parallax-derived distances are from Reid et al. (2014). To estimate a distance uncertainty for the EGOs that only have kinematic distances, we assessed the percent difference between the predicted kinematic distance and the parallax distance for the five sources for which both are available, and found a median percent difference of $15 \%$.

The median $L / M$ for our sources is $24.7 \pm 8.4 L_{\odot} / M_{\odot}$, where the uncertainty is the MAD and the $L / M$ for each source is the median of the four values shown in Figure 6 Most $L / M$ values fall in the range $5-60 L_{\odot} / M_{\odot}$, regardless of the method of deriving the luminosity. This result is in line with the results of Carpenter et al. (1990), who studied a set of 21 molecular clouds in the Outer Milky Way whose masses, luminosities, and suspected evolutionary state are comparable to our sample. They report an $L / M$ range of $1.1-39.2$ $L_{\odot} / M_{\odot}\left(\right.$ mean $\left.6.8 L_{\odot} / M_{\odot}\right)$. Typical L/M values for low- and intermediate-mass protostars, in contrast, usually span $\sim 0.1$ $-10 L_{\odot} / M_{\odot}$ (see, e.g., $L$ and $M$ values in Enoch et al. 2009).

For most of our sources, the four $L / M$ values we derive span a range of a factor of $\sim 2.5$, likely due to differences in the four different methods of deriving luminosity. The exceptions are G10.29-0.13 and G35.03+0.35, which span ranges of a factor of $\sim 100$ and $\sim 3$, respectively. The spread in $L / M$ 
for G10.29-0.13 is likely due to poorly-constrained SEDs, as has been previously discussed. G35.03+0.35 is discussed in the context of evolutionary stage in greater detail below.

The only notable trend in our $L / M$ values is the tendency of the Zhang \& Tan (2018) models to give $L / M$ values that are either higher than all three other methods, or lower than all three, but never in between. In particular, the Zhang \& $\operatorname{Tan}$ (2018) results tend to produce lower $L / M$ only when the value is very low $\left(<20 L_{\odot} / M_{\odot}\right)$; otherwise, the Zhang \& Tan (2018) $L / M$ is higher than both the two Robitaille- and the greybody-derived $L / M$ values. This dichotomy is entirely consistent with the trend in Stefan-Boltzmann luminosities for the Zhang \& Tan (2018) results noted in $\$ 4.3 .2$ We do not note any particular trend in $L / M$ with mass, though it should be noted that the mass range of our sample is small compared to that of other teams (Urquhart et al. 2018; Elia et al. 2017).

A comparison of our results with the results of other teams shows that our $L / M$ are well in line with established values for MYSOs. Both Urquhart et al. (2018) and Elia et al. (2017) compare $L$ and $M$ values for pre- and protostellar clumps. In both samples, massive star-forming regions are distinguished from prestellar sources by the space they occupy in $L-M$ parameter space. Urquhart et al. (2018) note that the $L / M$ values of massive star-forming clumps (as distinct from less massive or prestellar objects) are well-described by lower and upper limits of 1 and 100, respectively. Most $L / M$ in our sample fall in the range 5-60 $L_{\odot} / M_{\odot}$ (see Figure 6), which is well in line with the star-forming samples of both Urquhart et al. (2018) and Elia et al. (2017). Urquhart et al. (2018) further note that compact $\mathrm{H}$ II regions become common in their sample at $L / M>40$. The median $L / M$ for our sample is $\sim 25$, with a few $L>40$ from SED fit results. We have only one source which has $L>40$ for all four luminosities, G35.03+0.35, and this source does have a known UC H II region within the extended $4.5 \mu \mathrm{m}$ emission of the EGO.

Similarly, Tigé et al. (2017) examine 46 high-mass preand protostellar cores in NGC 6334 as part of the HershelHOBYS program. They separate their massive dense cores (MDCs) into three categories: IR-bright MDCs, IR-quiet MDCs, and Starless MDC Candidates. To distinguish IRbright and IR-quiet sources, they use flux limits of 10,12, and $15 \mathrm{Jy}$ at 21,22 , and $24 \mu \mathrm{m}$, respectively. Sources with fluxes above these limits are considered IR-bright, and sources with fluxes below these limits are IR-quiet. The $F$ values are based on the predicted mid-IR emission of a B3-type protostar and calculated for a distance of $1.75 \mathrm{kpc}$, which is the distance to NGC6334 assumed by Tigé et al. (2017). These values are consistent with the weak-to-bright cutoff used by König et al. (2017) when scaled to $4 \mathrm{kpc}$ (see $\$ 8$ ). Tigé et al. (2017) determine their source masses by fitting source SEDs from 70 to $1200 \mu \mathrm{m}$ using data from Herschel, JCMT, APEX, and SEST. In cases of no significant mid-IR emission, Tigé et al. (2017) determine bolometric luminosity by performing greybody fits to the far-IR data, similar to the process we use for our own data (see $\S 4.1$ ). In cases of significant mid-IR emission, they determine bolometric luminosity by integrating directly under the observed flux values. In this case, they use the data sets described above as well as data from 3.6 to $24 \mu \mathrm{m}$ from Spitzer, MSX, and WISE. Tigé et al. (2017) at all times assume optically thin emission at $\lambda>100 \mu \mathrm{m}$ and use $\beta=2$.

When we compare our sources with the Tigé et al. (2017) subsamples, an interesting feature emerges. Our median $L / M$ when $L$ is the greybody luminosity is $19.4 \pm 7.3 L_{\odot} / M_{\odot}$, and the median $L / M$ when $L$ is the median of all four luminosities is $24.7 \pm 8.4 L_{\odot} / M_{\odot}$. Compare this to the median $L / M$ of the IR-quiet and IR-bright cores in the Tigé et al. (2017) sample $\left(3.1 \pm 2.8 L_{\odot} / M_{\odot}\right.$ and $70 \pm 28 L_{\odot} / M_{\odot}$, respectively, where these medians are calculated from the $L_{\star}$ and $M_{\star}$ listed in Tigé et al. (2017), Table 3 ). The median $L / M$ of our sample falls neatly between the median $L / M$ of the IR-quiet and IR-bright populations in Tigé et al. (2017), whether we use $L$ from only the greybody fit or the median of all four $L$-values. Even with uncertainties, our sources are still well-separated from either category. While there are some differences between our methods of deriving $L / M$ and those of Tigé et al. (2017), these are unlikely to significantly change this result. Recalculating our $L$ and $M$ values using $\beta=2$ (the value used by Tigé et al. (2017)) instead of $\beta=1.7$ only increases our median $L / M$ by $7.3 \%$. This finding is consistent with Tigé et al. (2017), who calculate that using $\beta=1.5$ instead of $\beta=2$ would only alter their calculated masses by $5-10 \%$.

Tigé et al. (2017) suggest that IR-quiet MDCs are precursors to IR-bright MDCs; once IR-quiet cores have accreted enough mass to produce a stellar embryo with $\mathrm{M}>8 \mathrm{M}_{\odot}$, their luminosity sharply increases and they become IR-bright. (See Motte et al. (2018a) for a review of the theory and current observational support for this scenario.) This transition corresponds to the swelling phase of Hosokawa \& Omukai (2009), in which a massive protostar rapidly expands after reaching $M \sim 6 M_{\odot}$. The rapid expansion is driven by the sudden escape of significant entropy from the interior of the star, which can only occur after the opacity is sufficiently decreased by increasing temperature. The swelling phase is comparatively brief and lasts only until $M \sim 10 M_{\odot}$, and is immediately followed by a Kelvin-Helmholtz contraction phase in which the protostellar radius decreases again. Given that our median $L / M$ fall between the values for the wellestablished "IR-quiet" and "IR-bright" categories of massive protostellar objects, it is possible that our EGO-12 sample represents a transitional stage between the IR-quiet and IRbright phases of evolution, i.e., a phase in which an accreting protostar reaches some critical (large) mass, undergoes a concrete physical change, and consequently increases sharply in luminosity. Given the lack of predicted observable properties for this swelling/growth phase other than an increase in luminosity, we are hesitant to suggest that this is definitively the state in which our sources exist. However, the possibility is intriguing and suggests an interesting avenue for further investigation.

If $L / M$ is indeed a reliable indicator of evolutionary state, as asserted by other teams, then it should be unsurprising both that a) the majority of our sample, which were specifically selected due to their uniformity of evolutionary state, all exhibit very similar $L / M$ values, and b) that G35.03+0.35, which compared to the majority of the sample is in a very late stage of evolution, has a significantly higher $L / M$. Such a possibility - that EGOs in particular represent the stage of MYSO evolution immediately prior to the emergence of strong midinfrared emission and subsequent $\mathrm{H}$ II regions - warrants further investigation in future work.

\section{CONCLUSIONS \& FUTURE WORK}

We have conducted a multiwavelength study of twelve typical massive protoclusters in the Milky Way using SOFIA FORCAST imaging and archival infrared data. We performed aperture photometry at each wavelength in order to construct SEDs from the near-IR $(3.6 \mu \mathrm{m})$ to sub-mm $(870 \mu \mathrm{m})$, which we then fit with one greybody and three radiative transfer 


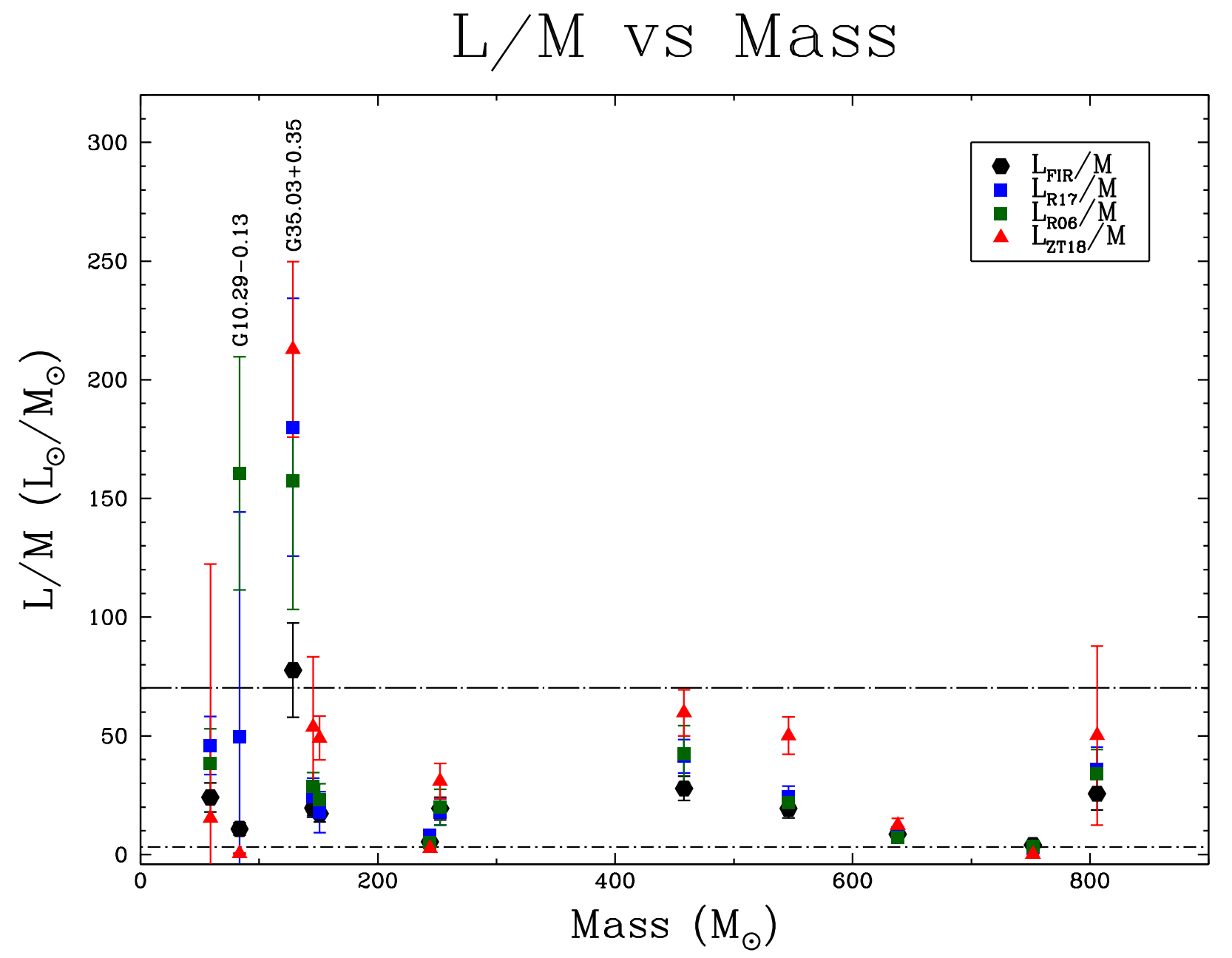

FIG. 6.- $L / M$ versus $M$ for all sources using all four luminosity values. Mass is the average of the $\mathrm{NH}_{3}$-derived and greybody-derived masses for each source. Symbols denote which luminosity value was used for L/M: black hexagons are the greybody-derived $L_{F I R}$, blue squares are the luminosity returned by the Robitaille (2017) models, green squares are the luminosity returned by the Robitaille et al.(2006) models, and red triangles are the luminosity returned by the Zhang \& Tan (2018) models. The upper and lower dash-dotted lines are the median $L / M$ values for the Tigé et al. (2017) "IR-bright" and "IR-quiet" categories, respectively. Sources G10.29-0.13 and G35.03+0.35 are labeled specifically, as they both have some $L / M$ values approximately an order of magnitude higher than the rest of our sample.

models. The radiative transfer models (Robitaille et al. 2006; Robitaille 2017; Zhang \& Tan 2018) all model near-IR to submillimeter emission in the context of a single protostar.

The SOFIA images, in conjunction with archival data, suggest that the number of massive sources per EGO is between 0.9 and 1.9. This moderate MYSO multiplicity is in line with published values for similar samples (Rosero et al. 2018) and for G11.92-0.61 (Cyganowski et al. 2017; Ilee et al. 2018). The multiplicity of these sources cannot be further constrained without sub-arcsecond resolution images, in either the mid-infrared or radio regimes. Cyganowski et al. (2017) do detect a plethora of lower-mass sources in G11.92-0.61, none of which are indicated in the SOFIA images; this is consistent with both our angular resolution limitations and with the luminosity sensitivity limit of $1.1 \times 10^{3}$ that we calculate in $\$ 4.3 .1$ which indicates these SOFIA observations will not be sensitive to lower-mass, lower-luminosity YSOs.

We find that, for this sample, the temperatures derived from greybody fits to dust emission are quite similar to the tem- peratures derived from single-component fits to $\mathrm{NH}_{3}(1,1)$ to $(3,3)$ emission (Cyganowski et al. 2013), with the dust temperatures trending slightly higher than the $\mathrm{NH}_{3}$ temperatures. While these differences fall below a level of statistical significance, this trend is in line with the published results of other teams (Giannetti et al. 2017; König et al. 2017) which find that, at temperatures above $\sim 15 \mathrm{~K}, \mathrm{NH}_{3}$ emission tends to probe gas that is slightly cooler than the local dust. We find that the median $T_{\text {dust }}$ of the EGO-12 sample is consistent with the median $T_{\text {dust }}$ of the Top100 sample (König et al. 2017), and most closely aligned with either the "IR-weak" or "IR-bright" subcategories. The overlap between the EGO $T_{\text {dust }}$ range and both the IR-weak and IR-bright $T_{\text {dust }}$ ranges suggests that EGO-12 sample may represent the cooler end of the IR-bright population, or possibly a separate, intermediate population between the IR-weak and IR-bright subcategories. High-precision temperature measurements over a much larger EGO sample are needed in order to address both possibilities.

The $L$ we derive from the greybody fits agree within $20-50 \%$ of other published $L$ for these sources (Moscadelli et 
al. 2016; Urquhart et al. 2018). We find that the greybodyderived $L$ of Urquhart et al. (2018) are greater than our greybody-derived luminosities in all cases. This is consistent with the fact that our greybody fits assume a single (cold) component, while Urquhart et al. (2018) use both a cold (greybody) and hot (blackbody) component for at least eleven of our sources. The Stefan-Boltzmann luminosities $\left(L_{\star}\right)$, which we calculate from the $R_{\star}$ and $T_{\star}$ returned by the radiative transfer models and which do account for hot-component emission, are typically of order $2 \times$ higher that the luminosities returned by our greybody fits. They are also more in line (within $\sim 30 \%$ rather than $50 \%$ ) with the luminosities of (Urquhart et al. 2018).

We find that the individual $R_{\star}$ and $T_{\star}$ returned by the three radiative transfer packages vary widely both within and between packages (one order of magnitude in $T_{\star}$ and up to two in $R_{\star}$ for both cases). This suggests that the models are having trouble converging to a single set of protostellar parameters, and that perhaps none of the model packages are fitting the sources uniquely well. This result is consistent with the fact that, although we assumed a single dominant source for modeling purposes, these sources are actually protoclusters rather than isolated protostars. Objects of different evolutionary states may be contributing to the total emission even if they are too embedded or too clustered to be detected individually in our SOFIA images. This possibility is further supported by the detection of multiple mid-infrared SOFIA sources within the ATLASGAL emission for most of our targets.

The specific cases of G11.92-0.61, G16.59-0.05, and G35.03+0.35 - for which high-resolution, high-sensitivity (sub)millimeter and/or radio-wavelength data are available - highlights the limitations of such (comparatively) lowresolution photometry when applied to clustered sources. In particular, it strongly suggests that SED model results should not be used to identify or describe the properties of disk candidates in high-mass protostars as is commonly done for isolated low-mass protostars (e.g. Spezzi et al. 2013), or should only be used with extreme caution or in cases where the isolation of the high-mass protostar can be positively confirmed. For clustered sources, lower-resolution infrared data can be a powerful tool for describing global properties of each protocluster and testing the multiplicity of massive sources, but results from models assuming individual protostellar sources should be used with caution.

The $L / M$ values of our sample are well in line with $L / M$ values measured by other teams (Carpenter et al. 1990), but fall between the two distinct IR-quiet and IR-bright categories suggested in Tigé et al. (2017). Given that the evolutionary stage in which our objects exist - MYSO outflows being powered by active protostellar accretion - is thought to be shortlived, it is possible that our sample represents the transitional stage between the IR-quiet and IR-bright phases of evolution. While intriguing, this possibility requires further investigation before any definitive statements can be made.

If $L / M$ is indeed indicative of evolutionary stage, then it likely correlates with other source properties such as outflow momentum, millimeter luminosity (e.g. as observed by ALMA, SMA, etc.), or the presence and nature of radio continuum emission. In order to assess the existence and strength of such correlations, additional centimeter-millimeter wavelength observations are needed. We have recently obtained or are in the process of obtaining ALMA 1.3 and $3.2 \mathrm{~mm}$ and JVLA 1.3 and $5 \mathrm{~cm}$ line and continuum observations for this purpose. These observations, and their correlation (or lack thereof) with the infrared and sub-mm results of this paper, will be presented in future publications.

\section{ACKNOWLEDGEMENTS}

We thank the referee for their timely, thoughtful review and helpful suggestions which have improved this paper. This work is based in part on observations made with the NASA/DLR Stratospheric Observatory for Infrared Astronomy (SOFIA). SOFIA is jointly operated by the Universities Space Research Association, Inc. (USRA), under NASA contract NNA17BF53C, and the Deutsches SOFIA Institut (DSI) under DLR contract 50 OK 0901 to the University of Stuttgart. Financial support for this work was provided by NASA through award \#BU 29120004 issued by USRA. This work is based in part on observations made with the Spitzer Space Telescope, which is operated by the Jet Propulsion Laboratory, California Institute of Technology under a contract with NASA. Herschel is an ESA space observatory with science instruments provided by European-led Principal Investigator consortia and with important participation from NASA. The ATLASGAL project is a collaboration between the Max-Planck-Gesellschaft, the European Southern Observatory (ESO) and the Universidad de Chile. It includes projects E-181.C-0885, E-078.F-9040(A), M-079.C-9501(A), M-081.C-9501(A) plus Chilean data. This research has made use of the NASA/ IPAC Infrared Science Archive, which is operated by the Jet Propulsion Laboratory, California Institute of Technology, under contract with the National Aeronautics and Space Administration. The National Radio Astronomy Observatory is a facility of the National Science Foundation operated under agreement by the Associated Universities, Inc. This research made use of NASA's Astrophysics Data System Bibliographic Services, APLpy, an open-source plotting package for Python (Robitaille and Bressert, 2012), the SIMBAD database (operated at CDS, Strasbourg, France), and CASA. CJC acknowledges support from the STFC (grant number ST/M001296/1).

Software- sedfitter, (Robitaille et al. 2006); sedfitter, (Robitaille 2017); sedfit, (Zhang \& Tan 2018); APLpy, (Robitaille \& Bressert 2012), http://aplpy.github.com 
APPENDIX

A: Robitaille (2017), ROBITAILlE ET AL. (2006), \& ZHANG \& TAN (2018) MODEL SED PLOTS FOR INDIVIDUAL SOURCES 

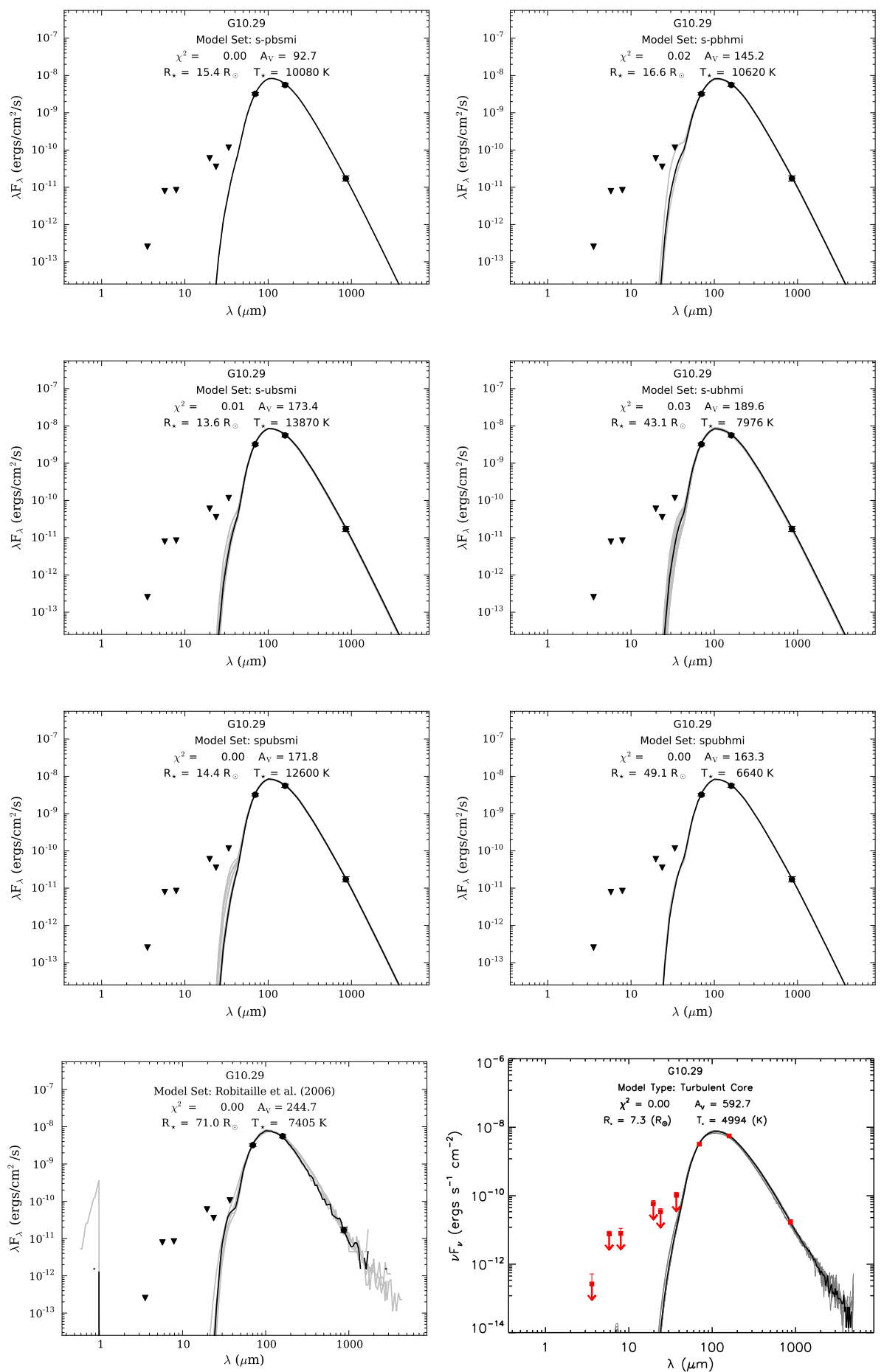

FIG. 7.- SED modeling results for G10.29-0.13, showing: (top six panels) the six best model sets from the Robitaille (2017) models based on $\chi^{2}$ values, (bottom left panel) the model results from Robitaille et al. (2006), and (bottom right panel) the model results from Zhang \& Tan (2018). 

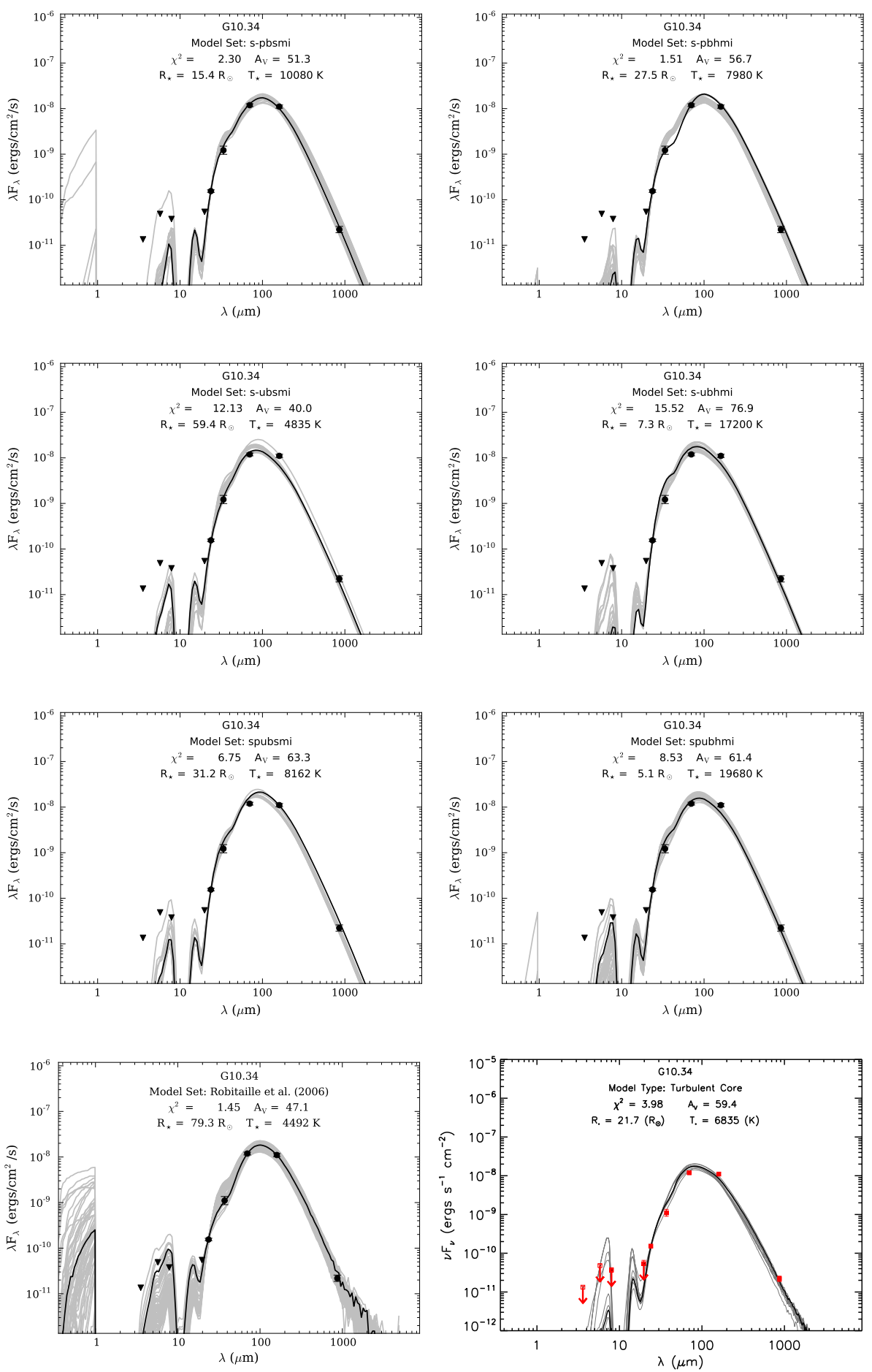

FIG. 8.- SED modeling results for G10.34-0.14, showing: (top six panels) the six best model sets from the Robitaille (2017) models based on $\chi^{2}$ values, (bottom left panel) the model results from Robitaille et al. (2006), and (bottom right panel) the model results from Zhang \& Tan (2018). 

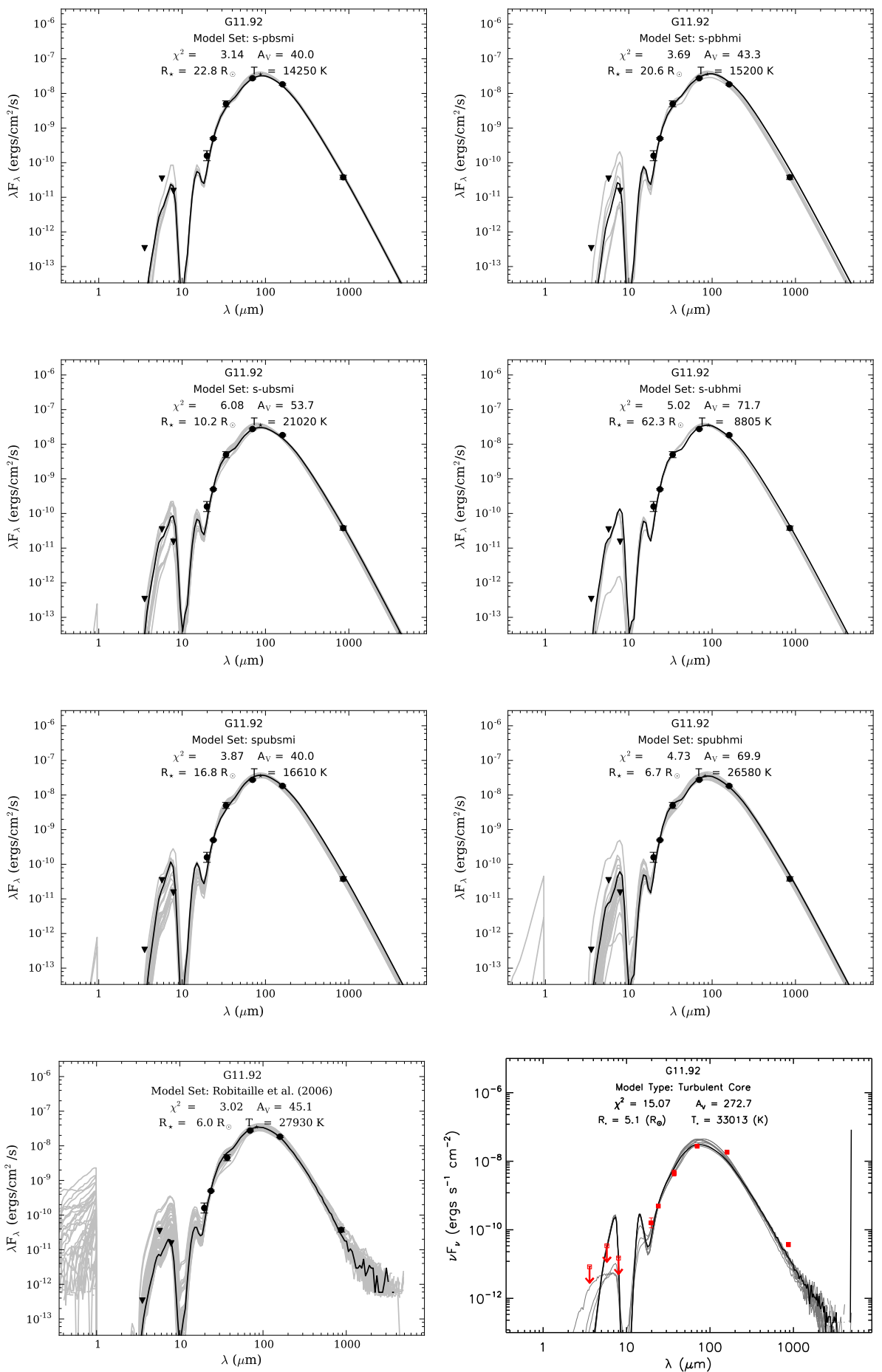

FIG. 9.- SED modeling results for G11.92-0.61, showing: (top six panels) the six best model sets from the Robitaille (2017) models based on $\chi^{2}$ values, (bottom left panel) the model results from Robitaille et al. (2006), and (bottom right panel) the model results from Zhang \& Tan (2018). 

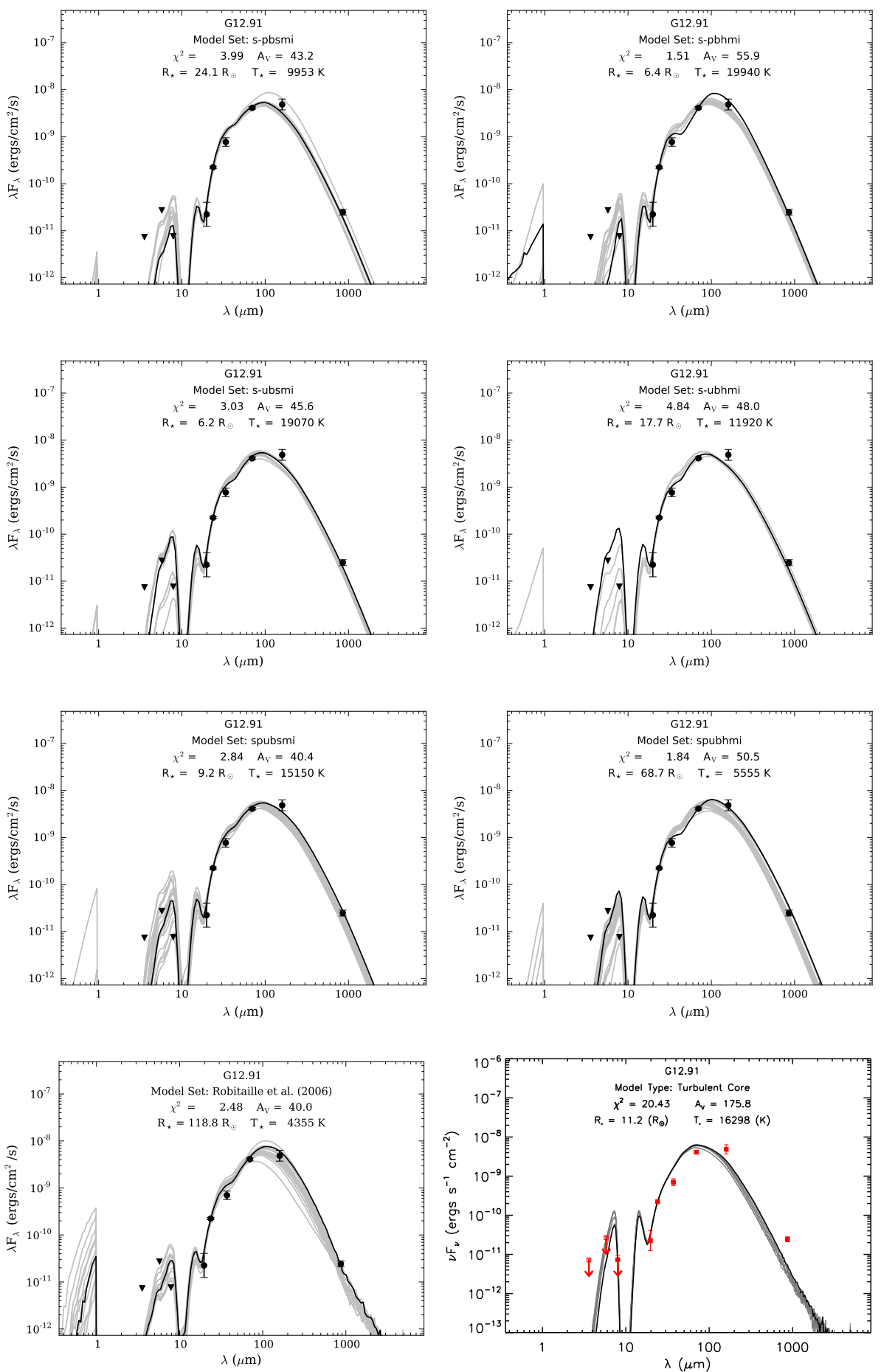

FIG. 10.- SED modeling results for G12.91-0.03, showing: (top six panels) the six best model sets from the Robitaille (2017) models based on $\chi^{2}$ values, (bottom left panel) the model results from Robitaille et al. (2006), and (bottom right panel) the model results from/Zhang \& Tan (2018). 

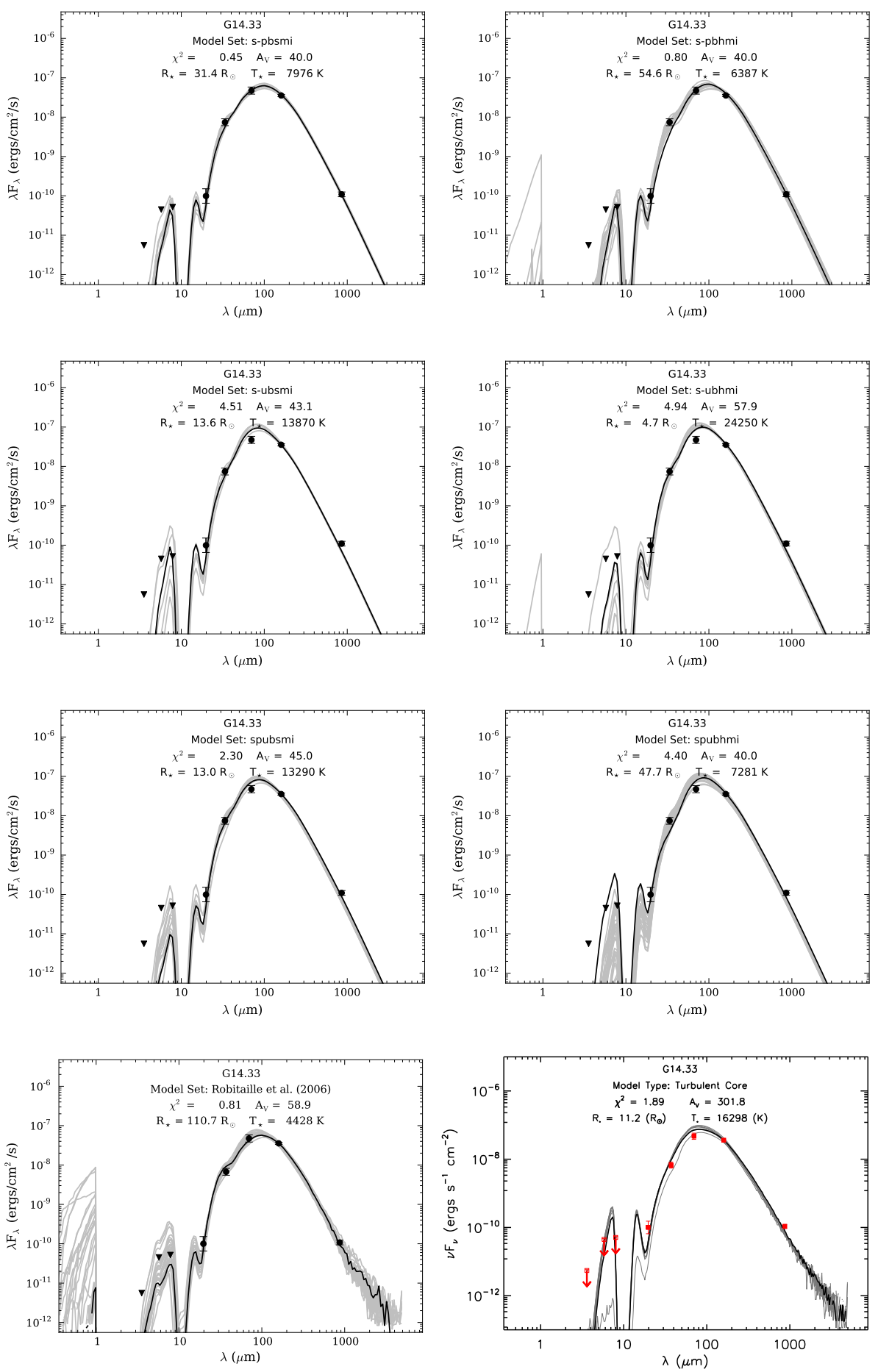

FIG. 11.- SED modeling results for G14.33-0.64, showing: (top six panels) the six best model sets from the Robitaille (2017) models based on $\chi^{2}$ values, (bottom left panel) the model results from Robitaille et al. (2006), and (bottom right panel) the model results from Zhang \& Tan (2018). 

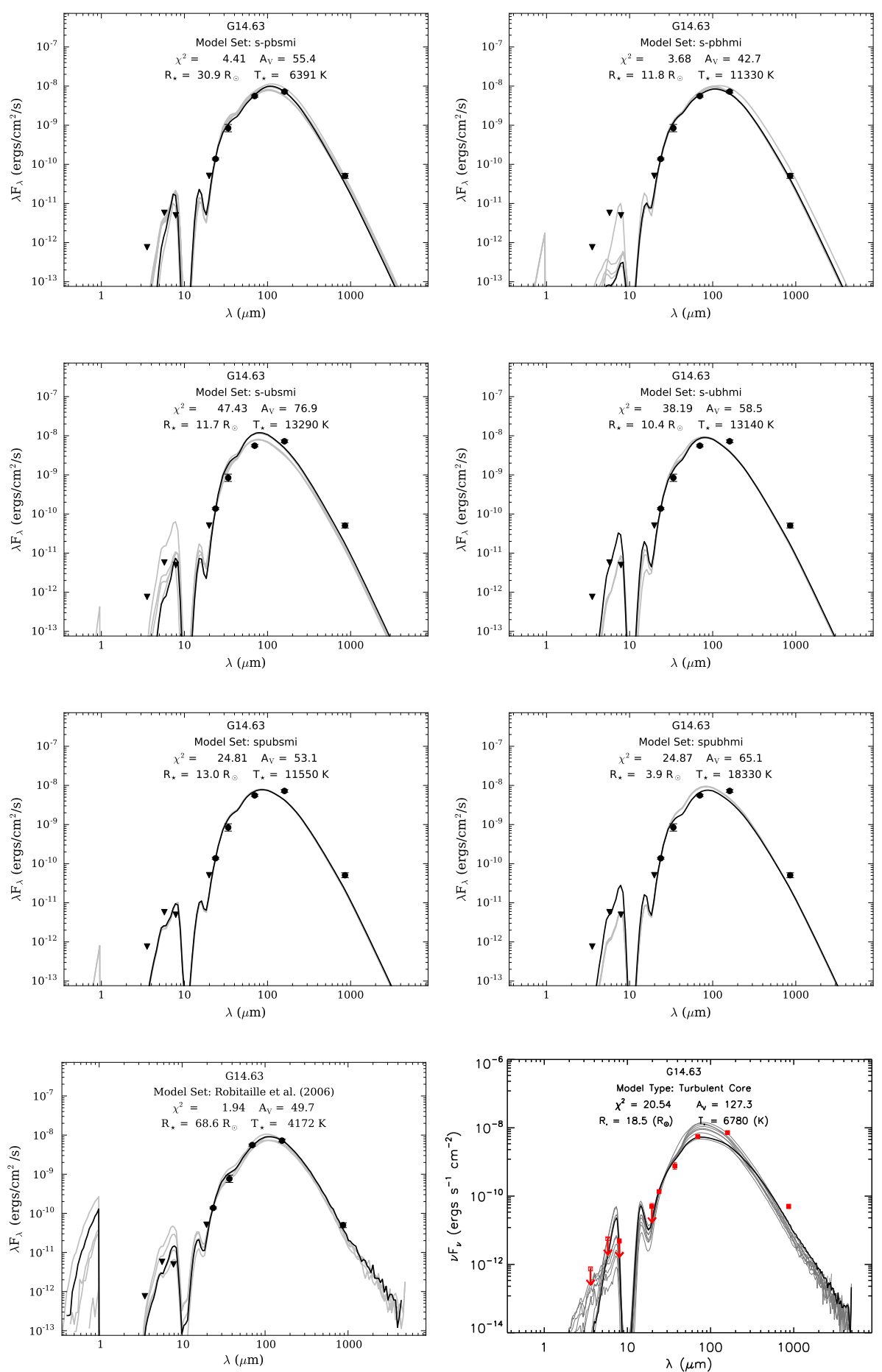

FIG. 12.- SED modeling results for G14.63-0.58, showing: (top six panels) the six best model sets from the Robitaille (2017) models based on $\chi^{2}$ values, (bottom left panel) the model results from Robitaille et al. (2006), and (bottom right panel) the model results from/Zhang \& Tan (2018). 

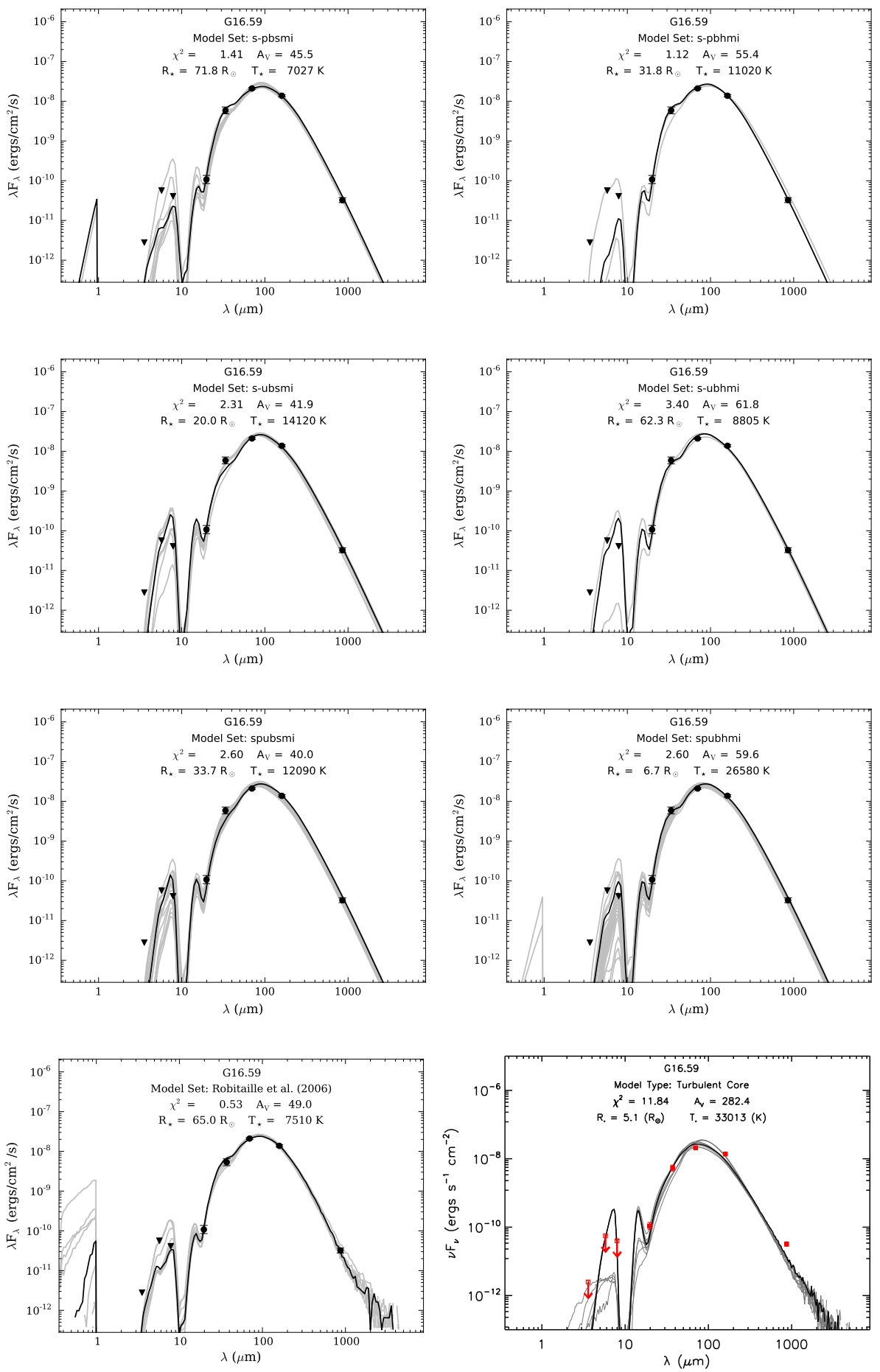

FIG. 13.- SED modeling results for G16.59-0.05, showing: (top six panels) the six best model sets from the Robitaille (2017) models based on $\chi^{2}$ values, (bottom left panel) the model results from Robitaille et al. (2006), and (bottom right panel) the model results from Zhang \& Tan (2018). 

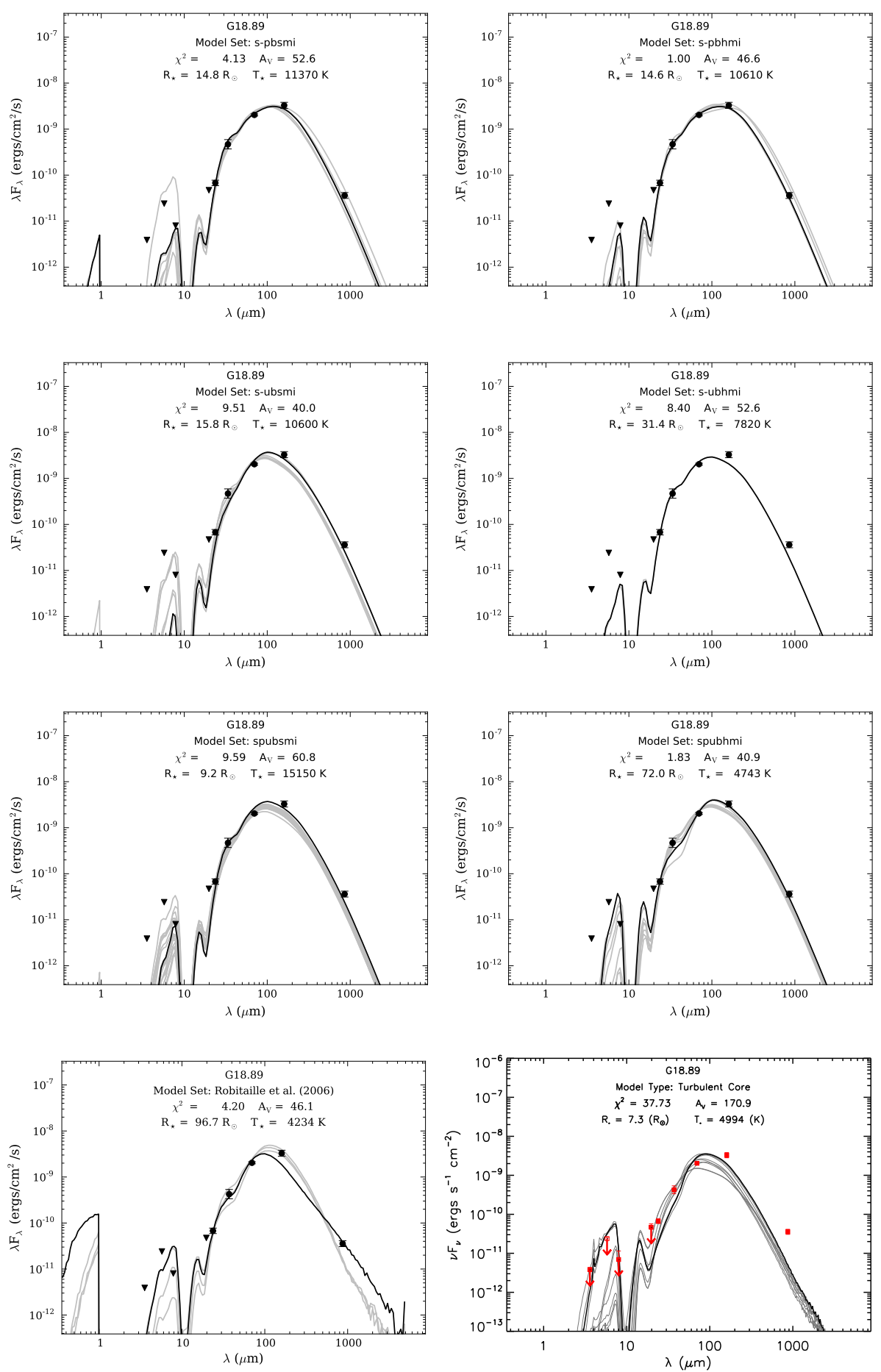

FIG. 14.- SED modeling results for G18.89-0.47, showing: (top six panels) the six best model sets from the Robitaille (2017) models based on $\chi^{2}$ values, (bottom left panel) the model results from Robitaille et al. (2006), and (bottom right panel) the model results from Zhang \& Tan (2018). 

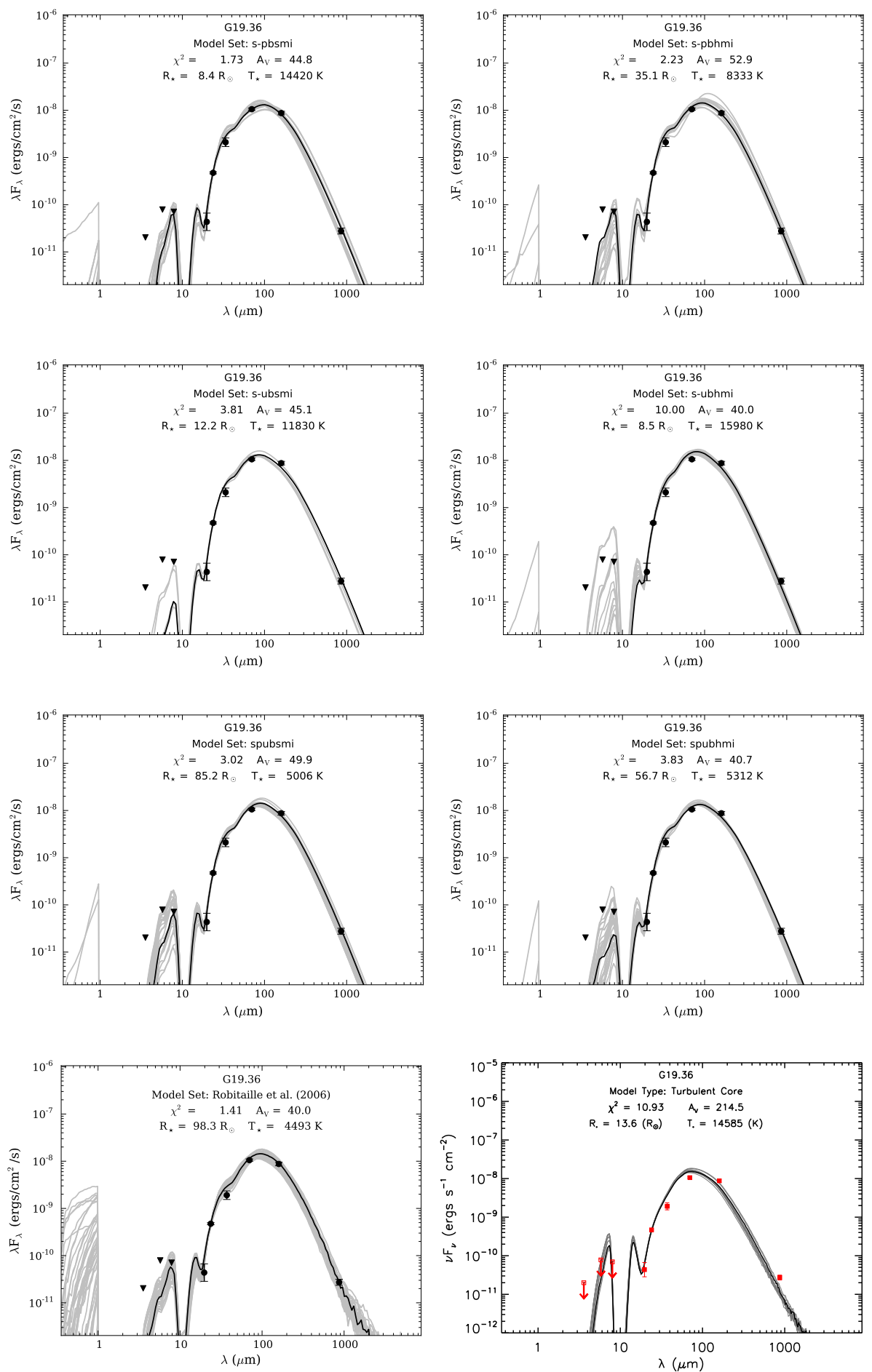

FIG. 15.- SED modeling results for G19.36-0.03 showing: (top six panels) the six best model sets from the Robitaille (2017) models based on $\chi^{2}$ values, (bottom left panel) the model results from Robitaille et al. (2006), and (bottom right panel) the model results from Zhang \& Tan (2018). 

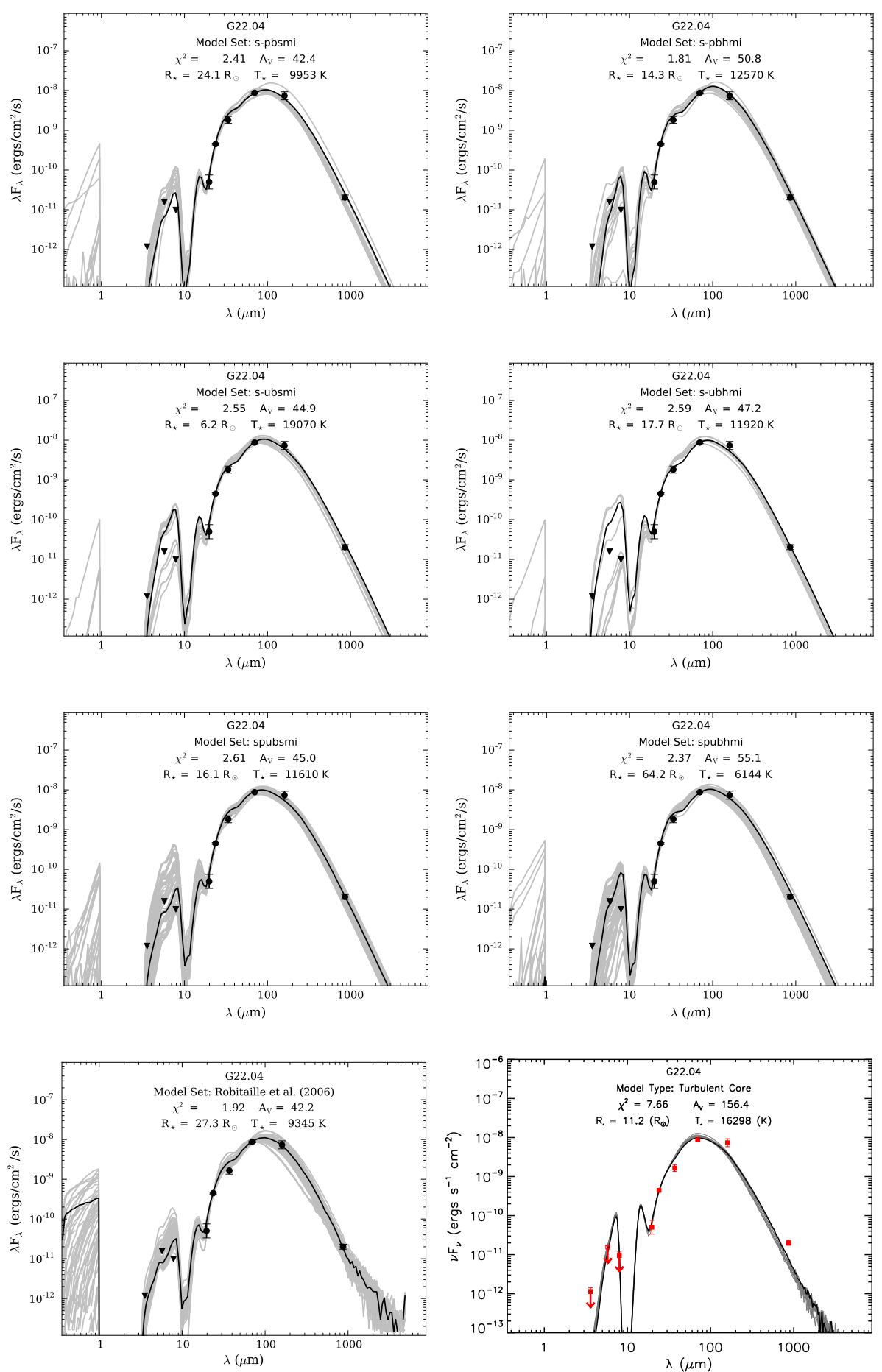

FIG. 16. - SED modeling results for G22.04+0.22, showing: (top six panels) the six best model sets from the Robitaille (2017) models based on $\chi^{2}$ values, (bottom left panel) the model results from Robitaille et al. (2006), and (bottom right panel) the model results from/Zhang \& Tan (2018). 

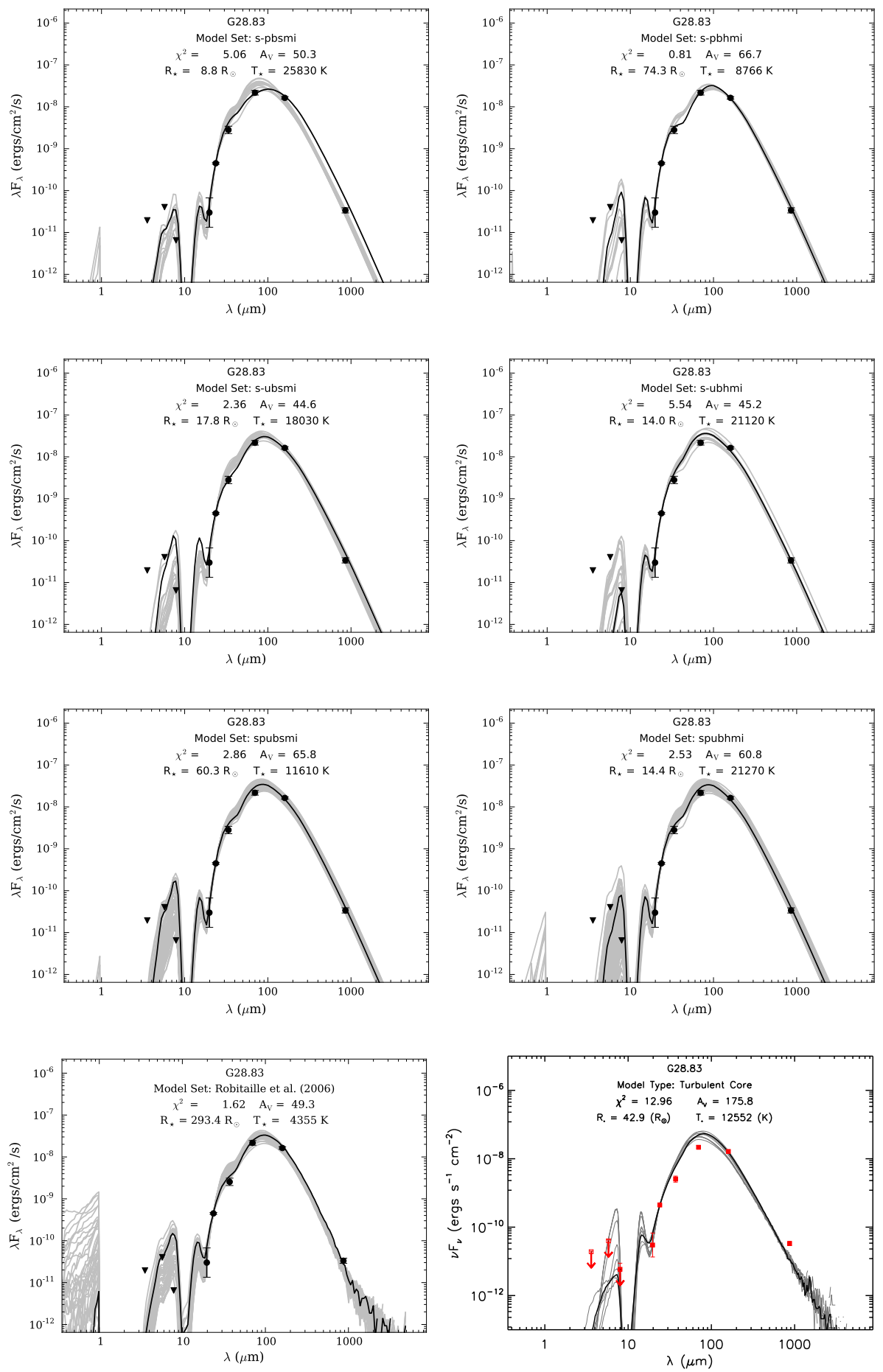

FIG. 17.- SED modeling results for G28.83-0.25, showing: (top six panels) the six best model sets from the Robitaille (2017) models based on $\chi^{2}$ values, (bottom left panel) the model results from Robitaille et al. (2006), and (bottom right panel) the model results from Zhang \& Tan (2018). 

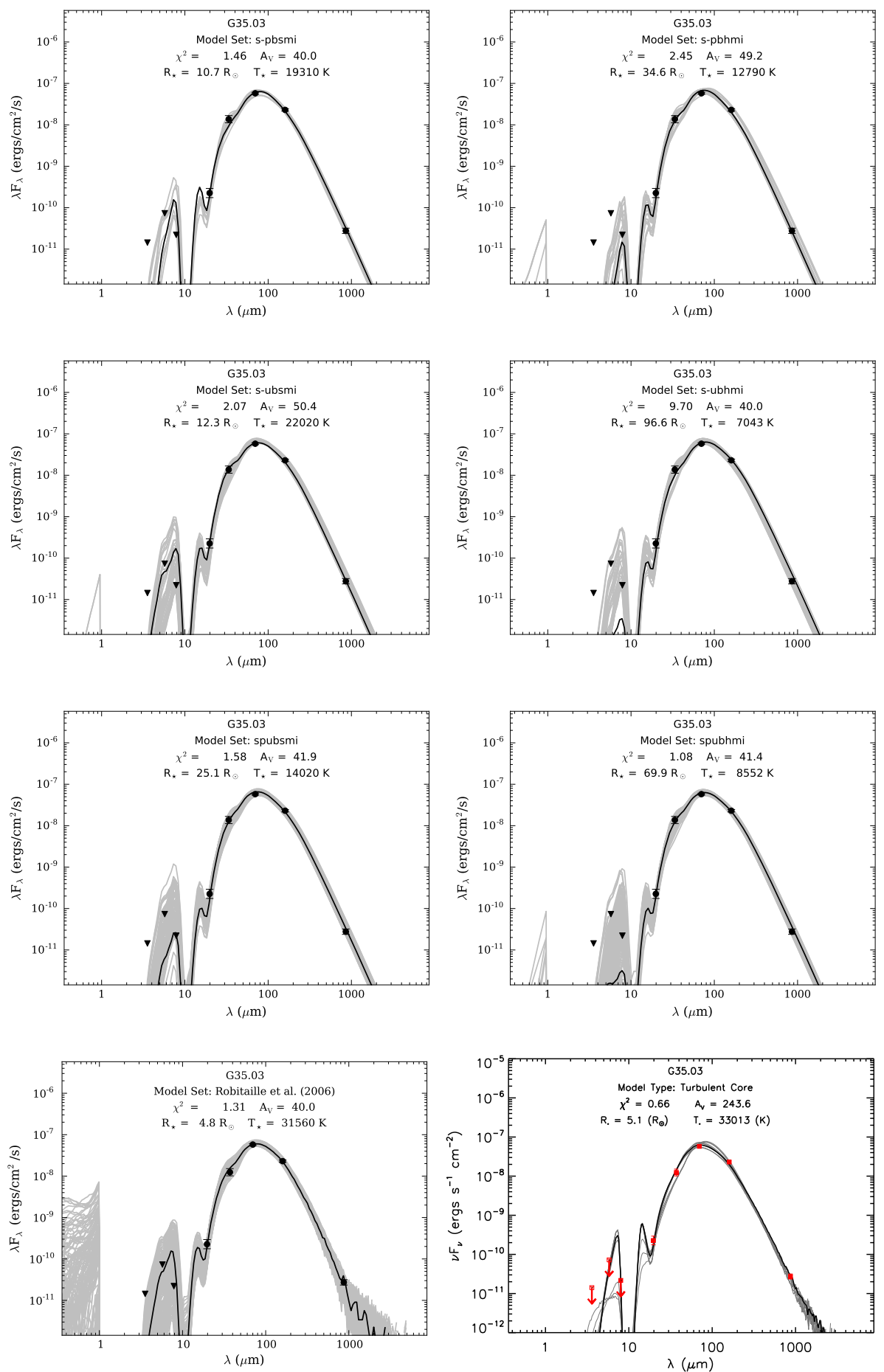

FIG. 18. - SED modeling results for G35.03+0.35, showing: (top six panels) the six best model sets from the Robitaille (2017) models based on $\chi^{2}$ values, (bottom left panel) the model results from Robitaille et al. (2006), and (bottom right panel) the model results from/Zhang \& Tan (2018). 
TABLE 10

$\chi^{2}$ And P(D|M) Scores For Robitaille (2017) Model Sets ${ }^{a, b}$

\begin{tabular}{|c|c|c|c|c|c|c|c|c|c|c|c|}
\hline $\begin{array}{l}\text { G10.29-0.13 } \\
\text { Model }\end{array}$ & $\chi^{2}$ & Model & $\mathrm{P}(\mathrm{D} \mid \mathrm{M})$ & $\begin{array}{l}\mathbf{G 1 0 . 3 4 - 0 . 1 4} \\
\text { Model }\end{array}$ & $\chi^{2}$ & Model & $\mathrm{P}(\mathrm{D} \mid \mathrm{M})$ & $\begin{array}{l}\text { G11.92-0.61 } \\
\text { Model }\end{array}$ & $\chi^{2}$ & Model & $\mathrm{P}(\mathrm{D} \mid \mathrm{M})$ \\
\hline spubhmi & 0.0003 & s-u-smi & 0.0519 & s-pbhmi & 1.51 & s-pbhmi & 0.0029 & s-pbsmi & 3.14 & s-ubsmi & 0.0015 \\
\hline spubsmi & 0.002 & spu-smi & 0.0422 & s-pbsmi & 2.30 & s-pbsmi & 0.0028 & s-pbhmi & 3.69 & s-pbsmi & 0.0009 \\
\hline s-pbsmi & 0.004 & spubhmi & 0.0408625 & spubsmi & 6.75 & spubhmi & 0.0008625 & spubsmi & 3.87 & s-pbhmi & 0.0007 \\
\hline s-ubsmi & 0.013 & s-pbhmi & 0.0384 & spubhmi & 8.53 & s-ubsmi & 0.0008 & spubhmi & 4.73 & spubsmi & 0.000675 \\
\hline s-u-smi & 0.017 & s-ubhmi & 0.0359 & s-ubsmi & 12.13 & s-ubhmi & 0.0359 & s-ubhmi & 5.02 & s-ubhmi & 0.0006 \\
\hline s-pbhmi & 0.022 & spubsmi & 0.02725 & s-ubhmi & 15.52 & spubsmi & 0.0325 & s-ubsmi & 6.08 & spubhmi & 0.0004125 \\
\hline s-ubhmi & 0.028 & s-pbsmi & 0.0261 & spu-smi & 21.70 & spu-smi & 0 & s-u-smi & 34.70 & spu-smi & 0 \\
\hline spu-smi & 0.044 & s-ubsmi & 0.0217 & s-u-smi & 23.52 & $\mathrm{~s}-\mathrm{u}-\mathrm{smi}$ & 0 & spu-smi & 40.68 & s-u-smi & 0 \\
\hline G12.91-0.03 & $\gamma^{2}$ & Model & $\mathrm{P}(\mathrm{D} \mid \mathrm{M})$ & G14.33-0.64 & $\gamma^{2}$ & Model & $\mathrm{P}(\mathrm{D} \mid \mathrm{M})$ & G14.63-0.58 & $\gamma^{2}$ & Model & $\mathrm{P}(\mathrm{D} \mid \mathrm{M})$ \\
\hline s-pbhmi & $\frac{x}{1.51}$ & s-pbhmi & $\frac{F(D \mid 1 / 1)}{0.0013}$ & s-pbsmi & $\frac{\chi}{0.45}$ & s-pbsmi & $\frac{F(D \mid \mathbf{D I})}{0.0034}$ & s-pbhmi & $\frac{\chi}{3.68}$ & s-pbsmi & $\frac{F(\mathrm{D} \mid \mathrm{VI})}{0.0007}$ \\
\hline spubhmi & 1.84 & s-ubsmi & 0.0013 & s-pbhmi & 0.80 & s-pbhmi & 0.003 & s-pbsmi & 4.41 & s-pbhmi & 0.0006 \\
\hline spubsmi & 2.84 & s-pbsmi & 0.0011 & spubsmi & 2.30 & s-ubhmi & 0.0006 & spubsmi & 24.81 & spubsmi & 0.0001 \\
\hline s-ubsmi & 3.03 & s-ubhmi & 0.0006 & spubhmi & 4.40 & s-ubsmi & 0.0006 & spubhmi & 24.87 & spubhmi & 0.00005 \\
\hline s-pbsmi & 3.99 & spubsmi & 0.00055 & s-ubsmi & 4.51 & spubhmi & 0.000425 & s-ubhmi & 38.19 & s-ubhmi & 0 \\
\hline s-ubhmi & 4.84 & spubhmi & 00003375 & s-ubhmi & 4.94 & spubsmi & 0.000425 & s-ubsmi & 47.43 & s-ubsmi & 0 \\
\hline s-u-smi & 28.58 & spu-smi & 0 & spu-smi & 12.78 & spu-smi & 0.0003 & spu-smi & 67.87 & spu-smi & 0 \\
\hline spu-smi & 31.37 & s-u-smi & 0 & s-u-smi & 12.84 & s-u-smi & 0.0002 & s-u-smi & 70.85 & s-u-smi & 0 \\
\hline $\begin{array}{l}\text { G16.59-0.05 } \\
\text { Model }\end{array}$ & $\chi^{2}$ & Model & $\mathrm{P}(\mathrm{D} \mid \mathrm{M})$ & $\begin{array}{l}\text { G18.89-0.47 } \\
\text { Model }\end{array}$ & $\chi^{2}$ & Model & $\mathrm{P}(\mathrm{D} \mid \mathrm{M})$ & $\begin{array}{l}\text { G19.36-0.03 } \\
\text { Model }\end{array}$ & $\chi^{2}$ & Model & $\mathrm{P}(\mathrm{D} \mid \mathrm{M})$ \\
\hline s-pbhmi & 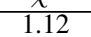 & s-pbsmi & 0.0011 & s-pbhmi & $\frac{\lambda}{1.01}$ & s-pbhmi & 0.0008 & s-pbsmi & 1.73 & s-pbsmi & 0.0022 \\
\hline s-pbsmi & 1.41 & s-ubsmi & 0.0008 & spubhmi & 1.83 & s-pbsmi & 0.0008 & s-pbhmi & 2.23 & s-ubhmi & 0.0019 \\
\hline s-ubsmi & 2.32 & spubsmi & 0.00045 & s-pbsmi & 4.13 & s-ubsmi & 0.0007 & spubsmi & 3.02 & s-pbhmi & 0.0015 \\
\hline spubsmi & 2.59 & spubhmi & 0.000425 & s-ubhmi & 8.40 & spubsmi & 0.000625 & s-ubsmi & 3.81 & spubsmi & 0.000625 \\
\hline spubhmi & 2.60 & s-pbhmi & 0.0003 & s-ubsmi & 9.51 & s-ubhmi & 0.0002 & spubhmi & 3.83 & s-ubsmi & 0.0005 \\
\hline s-ubhmi & 3.40 & s-ubhmi & 0.0003 & spubsmi & 9.59 & spubhmi & 0.0001 & s-ubhmi & 10.00 & spubhmi & 0.000425 \\
\hline spu-smi & 32.60 & spu-smi & 0 & spu-smi & 44.43 & spu-smi & 0 & s-u-smi & 20.86 & spu-smi & 0 \\
\hline s-u-smi & 35.47 & s-u-smi & 0 & s-u-smi & 44.73 & s-u-smi & 0 & spu-smi & 22.85 & s-u-smi & 0 \\
\hline $\begin{array}{l}\text { G22.04+0.22 } \\
\text { Model }\end{array}$ & $\chi^{2}$ & Model & $\mathrm{P}(\mathrm{D} \mid \mathrm{M})$ & $\begin{array}{l}\text { G28.83-0.25 } \\
\text { Model }\end{array}$ & $\chi^{2}$ & Model & $\mathrm{P}(\mathrm{D} \mid \mathrm{M})$ & $\begin{array}{l}\text { G35.03+0.35 } \\
\text { Model }\end{array}$ & $\chi^{2}$ & Model & $\mathrm{P}(\mathrm{D} \mid \mathrm{M})$ \\
\hline s-pbhmi & 1.81 & spubsmi & 0.001925 & s-pbhmi & 0.81 & spubsmi & 0.00255 & spubhmi & 1.08 & s-ubsmi & 0.0056 \\
\hline spubhmi & 2.37 & s-pbhmi & 0.0019 & s-ubsmi & 2.36 & s-ubsmi & 0.0023 & s-pbsmi & 1.46 & s-ubhmi & 0.0034 \\
\hline s-pbsmi & 2.41 & s-pbsmi & 0.0019 & spubhmi & 2.53 & s-ubhmi & 0.002 & spubsmi & 1.58 & spubhmi & 0.0033 \\
\hline s-ubsmi & 2.55 & s-ubsmi & 0.0019 & spubsmi & 2.86 & s-pbsmi & 0.002 & s-ubhmi & 1.94 & s-pbhmi & 0.0033 \\
\hline s-ubhmi & 2.59 & s-u-smi & 00017 & s-pbsmi & 5.06 & spubhmi & 0.00195 & s-ubsmi & 2.07 & spubsmi & 0.002775 \\
\hline spubsmi & 2.61 & spubhmi & 0.001025 & s-ubhmi & 5.54 & s-pbhmi & 0.0011 & s-pbhmi & 2.45 & s-u-smi & 0.0019 \\
\hline s-u-smi & 16.97 & s-ubhmi & 0.001 & s-u-smi & 18.12 & s-u-smi & 0.0006 & spu-smi & 9.47 & spu-smi & 0.0017 \\
\hline spu-smi & 19.13 & spu-smi & 0.0005 & spu-smi & 20.02 & spu-smi & 0 & s-u-smi & 12.47 & s-pbsmi & 0.0014 \\
\hline
\end{tabular}

a The $\chi^{2}$ values shown are $\chi^{2}$ per data point, as defined in $\$ 4.2$

${ }^{\mathrm{b}}$ Model sets are shown for each source in order of best (top row) to worst (bottom row). The first two columns for each source are the best model sets and $\chi^{2}$ values according to the $\chi^{2}$ method, and the second two columns for each source are the best model sets and $\chi^{2}$ values according to the Bayesian method. We show all eight model sets used in order to more clearly illustrate source trends according to both the $\chi^{2}$ and $\mathrm{P}(\mathrm{D} \mid \mathrm{M})$ methods - that is, to illustrate trends not only in which model sets produce the best fits, but also in which model sets produce the worst fits.

${ }^{c}$ In cases where the $\chi^{2}$ and $\mathrm{P}(\mathrm{D} \mid \mathrm{M})$ methods produce the same best-fit model set, source names and best-fit model set names are highlighted in bold.

\section{B: VARIATIONS IN THE RESULTS OF THE ROBITAILLE(2017) MODEL PACKAGES WITH BAYESIAN VERSUS $\chi^{2}$ BEST-FIT EVALUATIONS}

Robitaille (2017) stress that $\chi^{2}$ values alone may not be the optimal method of evaluating which source geometry best represents one's data. In order to assess the model sets in comparison to each other in a statistically robust way, Robitaille (2017) suggest calculating $\mathrm{P}(\mathrm{D} \mid \mathrm{M}) \propto N_{\text {good }} / N$, where $N_{\text {good }}$ is the total number of good models from a given model set, and $N$ is the total number of models in that set. This method accounts for the possibility that one model set may have produced an unusually low $\chi^{2}$ value that is not representative of the quality of that model set overall. In this approach, the model set with the highest number of good models is the best model set for a given source. The definition of "good" in this case is determined by the user; Robitaille (2017) use the convention $\chi^{2}-\chi_{\text {best }}^{2}<\mathrm{Xn}_{\text {data }}$, where $\chi_{\text {best }}^{2}$ is the best $\chi^{2}$ value across all model sets tested, $\mathrm{n}_{\text {data }}$ is the number of flux values that are not upper or lower limits, and $\mathrm{X}$ is a multiplicative factor chosen by the user. For the majority of our sources, $\chi^{2}$ $-\chi_{\text {best }}^{2}<3 \mathrm{n}_{\text {data }}$ gives a reasonable split between good and bad fits; the exception is G14.63-0.58, for which we used $X=5$ for reasons discussed below. Table 10 shows which model set best represents each source according to both the $\chi^{2}{ }_{\text {best }}$ and $\mathrm{P}(\mathrm{D} \mid \mathrm{M})_{\text {best }}$ methods of determining "best representation."

The $\chi^{2}$ and $\mathrm{P}(\mathrm{D} \mid \mathrm{M})$ approaches yield the same best-fit model set in five cases (G10.34-0.14, G12.91-0.03, G14.33-0.64, G18.89-0.47, and G19.36-0.03), and different model sets in seven. Sources for which both approaches yield the same best-fit model set have their source name and best model set name marked in bold in Table 10 . Interestingly, both the $\chi^{2}$ and Bayesian approaches tend to yield the same or very similar overall trends, such as, e.g., a strong preference for a power-law envelope or a 
slight preference for an inner hole. There are four sources for which the overall trends disagree on the presence or absence of at least one physical component (e.g. disk, inner hole); for one of these sources, the $\chi^{2}$ and Bayesian methods return results that differ on every physical component. It is worth noting that the four sources for which the trends identified by the $\chi^{2}$ and $\mathrm{P}(\mathrm{D} \mid \mathrm{M})$ methods show disagreement either lack a $24 \mu \mathrm{m}$ data point (the $24 \mu \mathrm{m}$ flux densities have the lowest uncertainties in $\lambda \mathrm{F}_{\lambda}$-space, so a $24 \mu \mathrm{m}$ non-detection has an outsize effect on the $\chi^{2}$ values for all models for that source) or suffer from confusion problems at $160 \mu \mathrm{m}$ as discussed in $\S 3.2 .3$. Interestingly, confusion problems at $70 \mu \mathrm{m}$ do not seem to produce similar disagreements in the model results.

Below, we discuss best-fit geometries for each source in detail, using both the $\chi^{2}$ and Bayesian methods of determining "best model."

G10.29-0.13 - The $\chi^{2}$ and Bayesian methods yield different best-fit model sets for this source (spubhmi for the former and s-u-smi for the latter), but both yield a general preference for a rotating-infalling (Ulrich-type) envelope and a slight preference for both a passive disk and no inner hole. All results for this particular source should be taken with the caveat that this source is quite poorly constrained (six of the nine flux densities used for SED modeling are upper limits).

G10.34-0.14 - Both methods yield the same trends for this source: a general preference for a power-law envelope and no passive disk for this source, with no real preference as to whether or not there is an inner hole. There is a significant increase (approximately a factor of 3) in $\chi^{2}$ values between the two lowest- $\chi^{2}$ model sets, which have no disk, and the third-best, which does. Likewise, there is a jump of approximately a factor of 3.5 in P(DIM) between the two best model sets (which do not have a passive disk) and the third-best, which does.

G11.92-0.61 - All preferred Robitaille (2017) model sets for G11.92-0.61 favor a power-law envelope and no disk, with no strong preference as to the presence or absence of an inner hole. This is true for both the $\chi^{2}$ and $P(D \mid M)$ methods of determining the best model set. The fact that the results favor not having a disk is in direct contradiction with our knowledge of this source from high-resolution centimeter- and millimeter-wavelength observations (see Ilee et al.|2016, 2018). This disagreement is likely

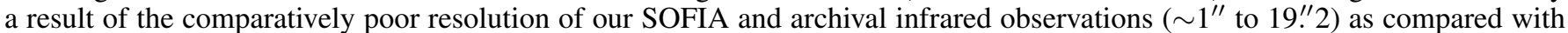
the millimeter observations ( $\sim 0 .{ }^{\prime \prime} 09$ to $\sim 0 . \prime 75$; Ilee et al. 2016, 2018); the infrared observations simply do not have sufficient resolution to distinguish the necessary small-scale structure in such a clustered source.

The discrepancy between the SED modeling results and the results of Ilee et al. (2016, 2018) warrant a closer look. Based on $\sim 0$." 5 (1550 au)-resolution Submillimeter Array (SMA) data, Ilee et al. (2016) estimated a disk gas mass of $\sim 2-3 M_{\odot}$ and

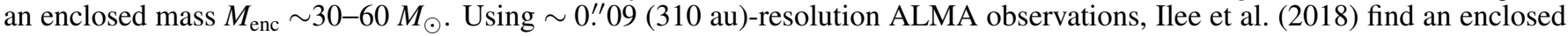
mass $M_{\text {enc }}$ of $40 \pm 5 M_{\odot}$ and a disk gas mass of $\sim 2-6 M_{\odot}$. The Robitaille et al. (2006) best-fit model returns a central source mass of $13.4 M_{\odot}$ and a disk gas mass of $4.6 \times 10^{-2} M_{\odot}$, while the Zhang \& Tan (2018) best-fit model gives a central source mass of $16 M_{\odot}$ and a disk mass of $5.33 M_{\odot}\left(\frac{1}{3}\right.$ the mass of the central source, as discussed in $\left.\$ 4.2 .3\right)$. In this case, while the Robitaille (2017) models do not favor the known physical geometry, the Robitaille et al. (2006) and Zhang \& Tan (2018) models do not reproduce the observationally-derived stellar and disk masses. That is, none of the three models accurately describes the known physical parameters of this source.

G12.91-0.03 - While the $\chi^{2}$ and $\mathrm{P}(\mathrm{D} \mid \mathrm{M})$ methods do produce the same best-fit model set for this source, the overall trends in the $\chi^{2}$ and $\mathrm{P}(\mathrm{D} \mid \mathrm{M})$ results disagree. Neither method particularly seems to favor one envelope type over another. However, the $\chi^{2}$ results overall favor models which have an inner hole and a passive disk, whereas the $\mathrm{P}(\mathrm{D} \mid \mathrm{M})$ evaluation shows a strong preference for having no disk, but no preference as to the presence or absence of an inner hole.

G14.33-0.64 - Both the $\chi^{2}$ values and $\mathrm{P}(\mathrm{D} \mid \mathrm{M})$ show a strong preference for having no disk and a power-law envelope, and no real preference as to the presence or absence of an inner hole.

G14.63-0.58 - Both methods show, for this source, a strong preference for no passive disk, and for a power-law envelope, with no real preference for or against an inner hole. The $\mathrm{P}(\mathrm{D} \mid \mathrm{M})$ results for this source should be considered carefully, however. G14.63-0.58 has few good fits in any model set, and so for this source we used the cutoff $\chi^{2}-\chi_{\text {best }}^{2}<5 \mathrm{n}_{\text {data }}$ instead.

G16.59-0.05 - The $\chi^{2}$ and P(D|M) evaluations both favor models with no inner hole and no disk, but differ as to envelope type. The $\chi^{2}$ values suggest that a power-law envelope produces the best fit to our data, while the Bayesian approach suggests an Ulrich-type envelope instead. G16.59-0.05 is saturated in the MIPSGAL data, so its SED lacks a flux density at that wavelength. MIR emission in YSOs tends to be dominated by emission from the protostellar envelope and/or outflow cavities, and our fitted $24 \mu \mathrm{m}$ flux densities were usually the best-constrained data points for a given source. It is possible that the discrepancy in preferred envelope type is due to this combination of factors.

As for the fact that both evaluations of the Robitaille (2017) results favor model sets with no disk, this is another source for which additional data in the literature show this implication to be incorrect. Moscadelli et al. (2016) identify G16.59-0.05 as a $\sim 20 M_{\odot}$ YSO with a disk/jet system. The central source appears as compact Ku- and K-band continuum emission at 0 .' 2 and 0 .' 1 resolutions, respectively, and the rotating disk is traced by multi-epoch EVN observations of $6.7 \mathrm{GHz}^{\mathrm{CH}} \mathrm{OH}_{3}$ masers associated with the compact $\mathrm{K} u$ - and K-band emission (for details of the EVN observations, see Moscadelli et al. 2016, and references therein). The jet is traced by extended C-band emission ( $\sim 6 \mathrm{~cm}, 0 . \prime 4$ resolution) in both Moscadelli et al. (2016) and Rosero et al. (2016).

Neither Moscadelli et al. (2016) nor Rosero et al. (2016) estimate disk mass or accretion rate, so we cannot asses the quality of the Robitaille et al. (2006) and Zhang \& Tan (2018) results in that context. However, unlike as for G11.92-0.61, both the 
Robitaille et al. (2006) and Zhang \& Tan (2018) models do give results for protostellar mass $\left(15 M_{\odot}\right.$ and $16 M_{\odot}$, respectively) that are fairly well in line with the mass reported by Moscadelli et al. (2016). While the specific parameters of the disk cannot be explored at this time, we can state that the Robitaille et al. (2006) and Zhang \& Tan (2018) $M_{\star}$ results are consistent, for the moment, with the results available in the literature.

G18.89-0.47 - Both methods of evaluation favor models with no disk and a power-law envelope for this source. However, the Bayesian approach suggests that models without an inner hole more accurately fit the data, whereas the $\chi^{2}$ values favor models that do have an inner hole. G18.89-0.47 is one of our sources with a confusion problem at $160 \mu \mathrm{m}$.

G19.36-0.03 - Both the $\chi^{2}$ and $\mathrm{P}(\mathrm{D} \mid \mathrm{M})$ values for this source strongly favor models with no disk, but neither shows any particular trend in envelope type or presence/absence of an inner hole.

G22.04+0.22 - Both methods of model evaluation agree for this source: models which have no disk and no inner hole are favored, but there is no strong preference as to envelope type.

G28.83-0.25 - The $\chi^{2}$ and $\mathrm{P}(\mathrm{D} \mid \mathrm{M})$ values for this source both favor models with an Ulrich-type envelope and no disk, with either no or a very slight preference for models with no inner hole.

G35.03+0.35 - This is the one source for which the $\chi^{2}$ and $\mathrm{P}(\mathrm{D} \mid \mathrm{M})$ values produce entirely different trends. While the model sets with the best $\chi^{2}$ values notably lack trends for any particular physical components, the $\mathrm{P}(\mathrm{D} \mid \mathrm{M})$ values show a strong preference for models with an Ulrich envelope, no disk, and an inner hole. This is the one source which has a known UCH II region in the EGO itself (as opposed to an H II region nearby but not within the ATLASGAL clump which hosts the EGO, as is the case with G14.33-0.64). 


\section{REFERENCES}

Banerjee, S., \& Kroupa, P. 2017, A\&A, 597, A28

Beltrán, M. T., Sánchez-Monge, Á., Cesaroni, R., et al. 2014, A\&A, 571, A52

Benjamin, R. A., Churchwell, E., Babler, B. L., et al. 2003, PASP, 115, 953

Bernasconi, P. A., \& Maeder, A. 1996, A\&A, 307, 829

Bonnell, I. A., Bate, M. R., Clarke, C. J., \& Pringle, J. E. 2001, MNRAS, 323, 785

Bonnell, I. A., Bate, M. R., \& Vine, S. G. 2003, MNRAS, 343, 413

Bonnell, I. A., Vine, S. G., \& Bate, M. R. 2004, MNRAS, 349, 735

Bonnell, I. A., \& Bate, M. R. 2006, MNRAS, 370, 488

Brogan, C. L., Hunter, T. R., Cyganowski, C. J., et al. 2011, ApJ, 739, L16

Brogan, C. L., Hunter, T. R., Cyganowski, C. J., et al. 2016, ApJ, 832, 187

Bronfman, L., Nyman, L.-A., \& May, J. 1996, A\&AS, 115, 81

Carey, S. J., Noriega-Crespo, A., Mizuno, D. R., et al. 2009, PASP, 121, 76

Carpenter, J. M., Snell, R. L., \& Schloerb, F. P. 1990, ApJ, 362, 147

Chen, X., Ellingsen, S. P., Shen, Z.-Q., Titmarsh, A., \& Gan, C.-G. 2011, ApJS, 196, 9

Chen, X., Ellingsen, S. P., He, J.-H., et al. 2012, ApJS, 200, 5

Chen, X., Gan, C.-G., Ellingsen, S. P., et al. 2013, ApJS, 206, 9

Churchwell, E., Babler, B. L., Meade, M. R., et al. 2009, PASP, 121, 213

Cyganowski, C. J., Brogan, C. L., \& Hunter, T. R. 2007, AJ, 134, 346

Cyganowski, C. J., Whitney, B. A., Holden, E., et al. 2008, AJ, 136, 2391-2412

Cyganowski, C. J., Brogan, C. L., Hunter, T. R., \& Churchwell, E. 2009, ApJ, 702, 1615

Cyganowski, C. J., Brogan, C. L., Hunter, T. R., \& Churchwell, E. 2011, ApJ, 743, 56

Cyganowski, C. J., Brogan, C. L., Hunter, T. R., Churchwell, E., \& Zhang, Q. 2011, ApJ, 729, 124

Cyganowski, C. J., Brogan, C. L., Hunter, T. R., et al. 2012, ApJ, 760, L20

Cyganowski, C. J., Koda, J., Rosolowski, E., et al. 2013, ApJ, 764, 61

Cyganowski, C. J., Brogan, C. L., Hunter, T. R., et al. 2014, ApJ, 796, L2 Cyganowski, C. J., Brogan, C. L., Hunter, T. R., et al. 2017, MNRAS, 468,

De Buizer, J. M., Liu, M., Tan., J. C., et al. 2017, ApJ, 843, 33

Di Francesco, J., Johnstone, D., Kirk, H., MacKenzie, T., \& Ledwosinska, E. 2008, ApJS, 175, 277

Draine, B. T. 2003a, ApJ, 598, 1017

Draine, B. T. 2003b, ARA\&A, 41, 241

Duarte-Cabral, A., Bontemps, S., Motte, F., et al. 2013, A\&A, 558, A125

Egan, M. P., Price, S. D., \& Kraemer, K. E. 2003, Bulletin of the American Astronomical Society, 35, 57.08

Elia, D., Molinari, S., Schisano, E., et al. 2017, MNRAS, 471, 100

Enoch, M. L., Evans, N. J., II, Sargent, A. I., \& Glenn, J. 2009, ApJ, 692, 973

Fazio, G. G., Hora, J. L., Allen, L. E., et al. 2004, ApJS, 154, 10

Fontani, F., Beltrán, M. T., Brand, J., et al. 2005, A\&A, 432, 92

Fontani, F., Cesaroni, R., \& Furuya, R. S. 2010, A\&A, 517, A56

Frank, A., Ray, T. P., Cabrit, S., et al. 2014, Protostars and Planets VI, 451

Gaczkowski, B., Preibisch, T., Ratzka, T., et al. 2013, A\&A, 549, A67

Green, J. A., Caswell, J. L., Fuller, G. A., et al. 2010, MNRAS, 409, 913

Giannetti, A., Wyrowski, F., Brand, J., et al. 2014, A\&A, 570, 65

Giannetti, A., Leurini, S., Wyrowski, F., et al. 2017, A\&A, 603, 33

Gutermuth, R. A., \& Heyer, M. 2015, AJ, 149, 64

Herter, T. L., Adams, J. D., De Buizer, J. M., et al. 2012, ApJ, 749, L18

Hosokawa, T., \& Omukai, K. 2009, ApJ, 691, 823

Hunter, T. R., Brogan, C. L., Megeath, S. T., et al. 2006, ApJ, 649, 888

Ilee, J. D., Cyganowski, C. J., Brogan, C. L., et al. 2018, arXiv:1811.05267

Ilee, J. D., Cyganowski, C. J., Nazari, P., et al. 2016, MNKAS, 462, 4386

Jaffe, D. T., Stier, M. T., \& Fazio, G. G. 1982, ApJ, 252, 601

Kang, H., Kim, K.-T., Byun, D.-Y., Lee, S., \& Park, Y.-S. 2015, ApJS, 221, 6

Kennicutt, R. C., \& Evans, N. J. 2012, ARA\&A, 50, 531
König, C., Urquhart, J. S., Csengeri, T, et al. 2017, A\&A, 599, 139

Kurtz, S., Churchwell, E., \& Wood, D. O. S. 1994, ApJS, 91, 659

Laor, A., \& Draine, B. T. 1993, ApJ, 402, 441

Markwardt, C. B. 2009, Astronomical Data Analysis Software and Systems XVIII, 411, 251

Marston, A. P., Reach, W. T., Noriega-Crespo, A., et al. 2004, ApJS, 154 333

McKee, C. F., \& Tan, J. C. 2003, ApJ, 585, 850

McMullin, J. P., Waters, B., Schiebel, D., Young, W., \& Golap, K. 2007. Astronomical Data Analysis Software and Systems XVI (ASP Conf. Ser

376), ed. R. A. Shaw, F. Hill, \& D. J. Bell (San Francisco, CA: ASP), 127

Molinari, S., Brand, J., Cesaroni, R., \& Palla, F. 1996, A\&A, 308, 573

Molinari, S., Pezzuto, S., Cesaroni, R., et al. 2008, A\&A, 481, 345

Molinari, S., Swinyard, B., Bally, J., et al. 2010, PASP, 122, 314

Molinari, S., Schisano, E., Elia, D., et al. 2016, A\&A, 591, A149

Mooney, T. J., and Solomon, P. M., 1988, ApJ, 334, L51

Moscadelli, L., Sánchez-Monge, Á., Goddi, C., et al. 2016, A\&A, 585, A71

Motte, F., Nony, T., Louvet, F., et al. 2018, Nature Astronomy, 2, 478

Motte, F., Bontemps, S., \& Louvet, F. 2018b, ARA\&A, 56, 41

Neugebauer, G., Habing, H. J., van Duinen, R., et al. 1984, ApJ, 278, L1

Ossenkopf, V., \& Henning, T. 1994, A\&A, 291, 943

Poglitsch, A., Waelkens, C., Geis, N., et al. 2010, A\&A, 518, L2

Reid, M. J., Menten, K. M., Brunthaler, A., et al. 2014, ApJ, 783, 130

Rieke, G. H., Young, E. T., Engelbracht, C. W., et al. 2004, ApJS, 154, 25

Robitaille, T. P., Whitney, B. A., Indebetouw, R., Wood, K., \& Denzmore, P. 2006, ApJS, 167, 256

Robitaille, T. P., Whitney, B. A., Indebetouw, R., \& Wood, K. 2007, ApJS, 169,328

Robitaille, T., \& Bressert, E. 2012, APLpy: Astronomical Plotting Library in

Python, Astrophysics Source Code Library. http://ascl.net/1208.017

Robitaille, T. P. 2017, A\&A, 600, A11

Rosero, V., Hofner, P., Claussen, M., et al. 2016, ApJS, 227, 25

Rosero, V., Tanaka, K. E. I., Tan, J. C., et al. 2018, arXiv:1809.01264

Russeil, D., Zavagno, A., Motte, F., et al. 2010, A\&A, 515, A55

Sadavoy, S. I., Stutz, A. M., Schnee, S., et al. 2016, A\&A, 588, A30

Sanna, A., Moscadelli, L., Beltrán, M., et al. 2019, A\&A, submitted

Schuller, F., Menten, K. M., Contreras, Y., et al. 2009, A\&A, 504, 415

Scoville, N. Z., and Good, J. C., 1989, ApJ, 339, 149

Siess, L., Dufour, E., \& Forestini, M. 2000, A\&A, 358, 593

Slysh, V. I., Val'tts, I. E., Kalenskii, S. V., \& Golubev, V. V. 1999, Astronomy Reports, 43, 785

Spezzi, L., Cox, N. L. J., Prusti, T., et al. 2013, A\&A, 555, A71

Sridharan, T. K., Beuther, H., Schilke, P., Menten, K. M., \& Wyrowski, F. 2002, ApJ, 566, 931

Tan, J. C., Beltrán, M. T., Caselli, P., et al. 2014, Protostars and Planets VI, 149

Temi, P., Marcum, P. M., Young, E., et al. 2014, ApJS, 212, 24

Tigé, J., Motte, F., Russeil, D., et al. 2017, A\&A, 602, A77

Towner, A. P. M., Brogan, C. L., Hunter, T. R., et al. 2017, ApJS, 230, 22

Urquhart, J. S., König, C., Giannetti, A., et al. 2018, MNRAS, 473, 1059

Val'tts, I. E., Ellingsen, S. P., Slysh, V. I., et al. 2000, MNRAS, 317, 315

Vázquez-Semadeni, E., González-Samaniego, A., \& Colín, P. 2017,

MNRAS, 467, 1313

Vig, S., Testi, L., Walmsley, M., et al. 2007, A\&A, 470, 977

Weingartner, J. C., \& Draine, B. T. 2001, ApJ, 548, 296

Westerhout, G. 1958, Bull. Astron. Inst. Netherlands, 14, 215

Wink, J. E., Altenhoff, W. J., \& Mezger, P. G., 1982, A\&A, 108, 227

Wright, E. L., Eisenhardt, P. R. M., Mainzer, A. K., et al. 2010, AJ, 140, 1868

Zhang, Q., Sridharan, T. K., Hunter, T. R., et al. 2007, A\&A, 470, 269

Zhang, Y., \& Tan, J. C. 2018, ApJ, 853, 18 